\title{
Catenaries in Viscous Fluid
}

\author{
Brato Chakrabarti
}

\author{
Thesis submitted to the Faculty of the \\ Virginia Polytechnic Institute and State University \\ in partial fulfillment of the requirements for the degree of
}

\author{
Master of Science \\ In \\ Engineering Mechanics \\ James Hanna, Chair \\ Shane D. Ross \\ Mark A. Stremler
}

May 5, 2015

Blacksburg, Virginia

Keywords: Catenary, String, Towing, Dynamical System, Phase Portrait, Viscous Flow

(C)2015, Brato Chakrabarti 


\title{
Catenaries in Viscous Fluid
}

\author{
Brato Chakrabarti
}

\begin{abstract}
Slender structures in fluid flow exhibit a variety of rich behaviors. Here we study the equilibrium shapes of perfectly flexible strings that are moving with a uniform velocity and axial flow in viscous fluid. The string is acted upon by local, anisotropic, linear drag forces and a uniform body force. Generically, the configurations of the string are planar, and we provide analytical expressions for the equilibrium shapes of the string as a first order five parameter dynamical system for the tangential angle of the body $(\theta)$. Phase portraits in the anglecurvature $\left(\theta, \partial_{s} \theta\right)$ plane are generated, that can be shown to be $\pi$ periodic after appropriate scaling and reflection operations. The rich parameter space allows for different kinds of phase portraits that give rise to a variety of curve geometries. Some of these solutions are unstable due to the presence of compressive stresses. Special cases of the problem include sedimenting filaments, dynamic catenaries, and towed strings. We also discuss equilibrium configurations of towed cables and other relevant problems with fixed boundary conditions. Special cases of the boundary value problem involve towing of neutrally buoyant cables and strings with pure axial flow between two fixed points.
\end{abstract}




\section{Acknowledgment}

Let me start by thanking my parents and kaka. Like always, they have been very supportive over the last two years. They have been with me through some of my difficult phases and have always kept their faith in me. It would not have been possible to finish this work without their love and encouragement.

I would like to thank my friends in India who have always been in touch with me and have helped me to cope up with a new place far from home. I would specially like to mention Arghya, Pratyaya, Ritesh, Sahu, Shreya, Spandy, Subhro and Sumit for their warmth and love.

Coming to Blacksburg, I would first like to thank Saikat da (now at OIST) and Pandey da (now at Twente). They have inspired me in many ways with my research. Hossain da and Souvick da have been great friends with whom I have shared all my concerns and problems. I shall also like to thank our former group member Ahmed for valuable discussions on some of the problems related to my thesis. My stay at Blacksburg was made memorable by some great friends like Leo, Nath, Padag, Santa and Wrik da.

I am grateful to my committee members Dr. Shane Ross and Dr. Mark Stremler. I took a class from Dr. Ross on dynamical systems and also had many discussions with him in his office. He has inspired me to study many problems that are not a part of this thesis. Dr. Stremler has been very supportive with my decisions and have always believed in me. He has pointed me to many interesting problems that I wish to study in future. I shall also like to thank Prof. Mark Paul who has inspired my thinking and approach towards science.

Finally I would like to thank the person without whom I would have not written a single word of this thesis, my advisor Dr. James Hanna. James has the biggest influence on me over the last two years. I had the opportunity to learn and know from him about geometry, mechanics and science. I have lost count of the number of different problems on varied topics that he has introduced me to. James has supported many of my decisions and has respected my choices and I am really thankful to him for that. Only I know how much I am going to miss him. I believe that he will guide and help me in future and will keep his door open for future scientific collaborations. 


\section{Contents}

1 Introduction and Background 1

$\begin{array}{lll}2 & \text { Formulation and generic solutions of the problem } & 5\end{array}$

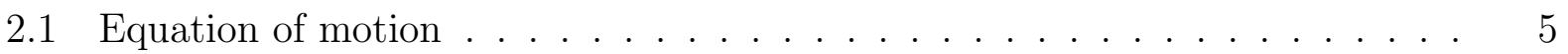

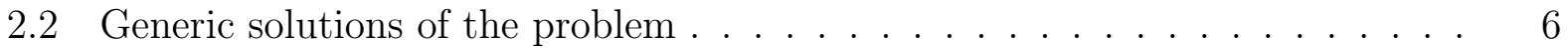

3 The planar problem and its special cases $\quad 9$

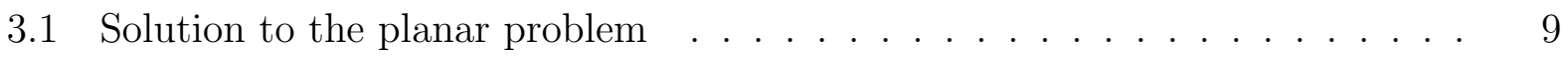

3.1 .1 Nondimensionalization . . . . . . . . . . . . . . . . . . 10

3.1.2 Obtaining a first order dynamical system . . . . . . . . . . . . 11

3.2 Representations of the dynamical system . . . . . . . . . . . . . . 13

3.2.1 Fixed Points, scaling and symmetries . . . . . . . . . . . . . 13

3.2 .2 Different phase portraits . . . . . . . . . . . . . . . . 16

3.2.3 Characteristics of curves arising from different phase trajectories . . 18

3.2.4 Numerical method for obtaining curves . . . . . . . . . . . . . . . . 19

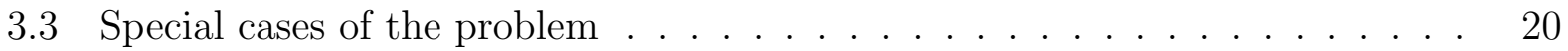

3.3 .1 Pure axial flow . . . . . . . . . . . . . . . . . . . 20

3.3.2 Straight line solutions from fixed points . . . . . . . . . . . . . 21

4 Parameter space visualization $\quad 22$

4.1 Octant of a sphere and its projection . . . . . . . . . . . . . 22 
4.2 Effect of changing the angle of translation $\phi \ldots \ldots \ldots \ldots$

4.3 Effect of changing $R_{\nu}$ on the parameter space $\ldots \ldots \ldots \ldots$

5 Heteroclinic Connections $\quad 30$

6 Sedimentation of flexible filaments $\quad 35$

$\begin{array}{lll}7 & \text { One end blow up and geometric special cases } & 39\end{array}$

7.1 One end blow up . . . . . . . . . . . . . . . . . 39

7.2 Geometric Special Cases . . . . . . . . . . . . . . . . . . . 40

7.2 .1 Phase portraits with doubled periodicity . . . . . . . . . . 40

$7.2 .2 \quad$ Circle and Logarithmic Spirals . . . . . . . . . . . . . . . . . 43

$\begin{array}{lll}8 & \text { Strange Behavior of the stress } & 47\end{array}$

8.1 Stress regions in the parameter space $\ldots \ldots \ldots \ldots . \ldots . \ldots 4$

8.2 Transition of the stress at the ends of a curve $\ldots \ldots \ldots 49$

9 Boundary value problems $\quad 51$

9.1 Towing of strings of fixed lengths $\ldots \ldots \ldots \ldots \ldots \ldots \ldots \ldots \ldots$

9.1.1 Finding the plane containing the curve . . . . . . . . . . 51

9.1 .2 Curves in $3 \mathrm{D} \ldots \ldots \ldots \ldots \ldots \ldots \ldots$

9.1 .3 Pure towing . . . . . . . . . . . . . . . . 55

9.2 Pure axial flow: reeling in and out . . . . . . . . . . . . 56

9.2 .1 Formulation . . . . . . . . . . . . . . . . . . 57

$9.2 .2 \quad$ Equilibrium shapes . . . . . . . . . . . . . . . . . 58

9.2 .3 Shapes for the special case . . . . . . . . . . . . . . . . . . . . . 59

9.2 .4 Stress at the trailing end . . . . . . . . . . . . 60

$\begin{array}{ll}10 \text { Discussion and future work } & 61\end{array}$ 
$\begin{array}{ll}\text { B Scaling between adjacent lobes } & 67\end{array}$

$\begin{array}{ll}\text { C On conditions for different phase portraits } & 69\end{array}$

C.1 Linearized Functions . . . . . . . . . . . . . . . . . . . . . . 70

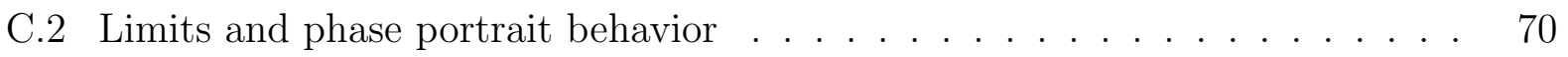

$\begin{array}{ll}\text { D Stress at the end of the string } & 73\end{array}$

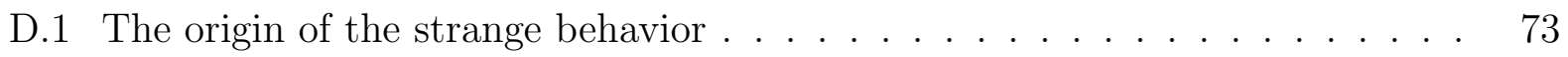

D.2 Relations governing the stress $\ldots \ldots \ldots \ldots \ldots \ldots$

$\begin{array}{ll}\text { Bibliography } & 77\end{array}$ 


\section{List of Figures}

1.1 Illustration from the solutions of Leibniz and Huygen ${ }^{[1]}$. Source: http://curvebank.calstatela.edu/ catenary/catenary.htm. Used under fair use, 2015. . . . . . . . . . . . . . . . . . . . . .

1.2 Examples of the catenary arising in nature. The first image is the The Gateway Arch at St. Louis, Missouri which is an example of a catenary arch. The second image is of a spider web where the filaments have assumed the shape of a catenary. The third image is that of a hanging chain, the classic example of a catenary. Source: http://en.wikipedia.org/wiki/Catenary. Used under fair use, 2015. . . . . . . . . . . . . . . . . . . .

1.3 A 1958 U.S. postage stamp. Source: https://en.wikipedia.org/wiki/Transatlantic_ telegraph_cable. Used under fair use, 2015. . . . . . . . . . . . . . . . . .

2.1 The string ............................ 5

3.1 Planar curve . . . . . . . . . . . . . . . . . . . . . . . 11

3.2 Heteroclinic connections as phase portrait. The figure shows three lobes of the phase portrait that go over an angle of $3 \pi$. Two fixed points have been marked on the figure. . . . . . . . . . . . . . . . . . . . . . . . . 14

3.3 Scaling and asymmetry in axial flow . . . . . . . . . . . . . . . 15

3.4 This figure shows three possible kinds of phase portraits that one can observe for different choices of parameters. . . . . . . . . . . . . . . . . 17

3.5 In the above figure, pink region corresponds to heteroclinic connections, the green region corresponds to one end blow up and the orange colored region corresponds to two end blow up. . . . . . . . . . . . . . . . . 
4.1 Different regions on the sphere for Stokes flow with angle of translation of the string being $-88^{\circ}$. Pink: Heteroclinic connections, Orange: Two end blow up, Green: One end blow up . . . . . . . . . . . . . . .

4.2 Projected view of the octant of the sphere on the $|\mathbf{v}|-q$ plane. This projection is for Stokes flow $\left(R_{\nu}=0.5\right)$ and the angle of translation is $-88^{\circ} . \ldots$.

4.3 Phase trajectories (left) and configurations (right) corresponding to the dotted locations in the central diagram. The translational velocity is nearly collinear with the body force $q$, with the angle of translation being $-89.9^{\circ}$. There is an additional tangential velocity $T=0.2$ in the arrowed directions and the anisotropy $R_{\nu}=0.5$ (Stokes flow). Dashed ends indicate that the configuration extends indefinitely, while a dot on the end of a curve indicates that the configuration terminates. . . . . . . . . . . . . . . . .

4.4 Orthographic projections of the positive octant of the sphere onto the $|\mathbf{v}|-q$ plane for different values of angle of translation at Stokes flow $\left(R_{\nu}=0.5\right)$. .

4.5 Different regions on the $|\mathbf{v}|-q$ plane for different values of drag ratio at a constant angle of translation, $\phi=-85^{\circ} \ldots \ldots \ldots \ldots$

5.1 Curves (left) and the phase trajectories with the stress distribution (right) for upward translation for Stokes flow $\left(R_{\nu}=0.5\right)$. Here $|\mathbf{v}|=0.1,0.5,0.95 ; q=$ $0.99,0.87,0.31 ; T=0$ and $m=1 \ldots \ldots \ldots$

5.2 Curves (left) and the phase trajectories with the stress distribution (right) for Stokes flow $\left(R_{\nu}=0.5\right)$. Here $|\mathbf{v}|=0.1,0.5,0.95 ; q=0.99,0.87,0.31 ; T=0$; $m=1$. Angle of translation $\phi=45^{\circ} \ldots \ldots \ldots$

5.3 Curves (left) and the phase trajectories with the stress distribution (right) for Stokes flow $\left(R_{\nu}=0.5\right)$. Here $|\mathbf{v}|=0.1,0.5,0.95 ; q=0.99,0.87,0.31 ; T=0$; $m=1$. The curves are translating in the horizontal direction $\left(\phi=0^{\circ}\right)$. . .

5.4 Curves (left) and the phase trajectories with the stress distribution (right) for Stokes flow $\left(R_{\nu}=0.5\right)$. Here $|\mathbf{v}|=0.1,0.5,0.95 ; q=0.99,0.87,0.31 ; T=0$; $m=1$. Angle of translation $\phi=-45^{\circ} \ldots \ldots \ldots$. . . . . . .

5.5 Curves (left) and the phase trajectories with the stress distribution (right) for Stokes flow $\left(R_{\nu}=0.5\right)$. Here $|\mathbf{v}|=0.1,0.5,0.95 ; q=0.99,0.87,0.31 ; T=0$; $m=1$. The velocity of translation is almost collinear with the body force with, $\phi=-90^{\circ} \ldots \ldots \ldots \ldots \ldots$ 
5.6 Curves (left) along with the phase portrait and thr stress distribution (right) for Stokes flow $\left(R_{\nu}=0.5\right)$. Here $|\mathbf{v}|=0.28,0.29,0.28 ; q=0.94,0.96,0.94$; $T=-0.2,0,0.2 ; m=1$. Angle of translation $\phi=-45^{\circ}$. For the cut through the parameter space we had, $\frac{|\mathbf{v}|}{q}=0.3 \ldots \ldots \ldots \ldots$

6.1 Sedimentation solution for purely flexible filaments (left) and stress distribution (bottom right), corresponding to the two end blow up phase trajectories (top right) at Stokes flow $\left(R_{\nu}=0.5\right)$. We have, $|\mathbf{v}|=0.74,0.75,0.76 ; q=$ $0.67,0.66,0.64$. Axial flow, $T=0, m=1$ and $\phi=-90^{\circ}$. The small circles at the end of the curves indicate that these curves are all of finite length in contrast to the infinite length curves obtained from the heteroclinic connections that has been shown by dashed line at the ends. . . . . . . . . . . .

6.2 Curve (left) and the orbit with the stress distribution (left) for two end blow up phase portraits in Stokes flow $\left(R_{\nu}=0.5\right)$. Here $|\mathbf{v}|=0.76, q=0.64$, $T=0.13$ and $m=1$. The angle of translation $\phi=-90^{\circ} \ldots \ldots \ldots$

7.1 Curve (left) and the orbit with the stress distribution (left) for one end blow up phase portraits for $R_{\nu}=0.6$. We have, $|\mathbf{v}|=0.78, q=0.57, T=0.25$ and $m=1$. The angle of translation $\phi=-90^{\circ} \ldots \ldots \ldots \ldots$

7.2 A looping curve (left) from $2 \pi$ periodic heteroclinic connection (top right) with the stress distribution (bottom right) for $R_{\nu}=0.4$. Here $T=0.2, \mathbf{v} \approx 0.82$, $q=0.53$ and $m=1$. The angle of translation, $\phi=-90^{\circ}$. . . . . . .

7.3 Finite length curve (left) obtained from $2 \pi$ periodic blowup phase portrait (top right) with the stress distribution (bottom right) for Stokes flow $\left(R_{\nu}=0.5\right)$. Here $T=0.1,|\mathbf{v}| \approx 0.78, q=0.61$ and $m=1$. The angle of translation $\phi=-90^{\circ}$.

7.4 The two points corresponding to the special case of spirals for Stokes flow $\left(R_{\nu}=0.5\right)$. The angle of translation $\phi=-88.5^{\circ} \ldots \ldots \ldots \ldots$

7.5 Logarithmic spiral as a special solution at Stokes flow $\left(R_{\nu}=0.5\right)$. Here we have: $T=0,|\mathbf{v}| \approx 0.79, q=0.61$ and $m=1$. The angle of translation $\phi=-88.5^{\circ}$.

7.6 Circle as a special solution at Stokes flow $\left(R_{\nu}=0.5\right)$. Here we have: $T=0$, $|\mathbf{v}|=0.8, q=0.6$ and $m=1$. The angle of translation $\phi=-90^{\circ} \ldots \ldots$ 
8.1 Different region of stress behavior in the octant of the sphere at Stokes flow $\left(R_{\nu}=0.5\right)$. The large dashed line corresponds to stress transition at the leading end $\left(l_{1}\right)$. The short dashed line corresponds to stress transition at the trailing end $\left(l_{2}\right)$. The angle of translation $\phi=-88^{\circ} \ldots \ldots . . . . .$.

8.2 Orthographic projection of the parameter space and the curves corresponding to the three points (left). Orbits along with the stress distributions (right) for $R_{\nu}=0.6$. Here, $T=0.25 ;|\mathbf{v}|=0.74,0.76,0.78 ; q=0.63,0.60,0.57 ; m=1$. The angle of translation $\phi=-89^{\circ}$. . . . . . . . . . . .

8.3 Orthographic projection of the parameter space and the curves corresponding to the three points (left). Orbits along with the stress distributions (right) for $R_{\nu}=0.6$. Here, $T=0.2 ;|\mathbf{v}|=0.84,0.88,0.93 ; q=0.49,0.41,0.30 ; m=1$. The angle of translation $\phi=-89^{\circ}$.

9.1 The figure shows two perspectives of how the plane containing the curve changes in $3 \mathrm{D}$ when it is towed from its end points with different velocities. Darker curves mean higher magnitude of velocity of translation. The parameters for the problem are: body force, $\overline{\mathbf{q}}=4$; velocities, $\overline{\mathbf{v}}=4,8,12$; the angle between the velocity vector and $\hat{\mathbf{R}}$ is $45^{\circ}$; the distance between end points, $|\mathbf{R}|=0.8$; normal drag coefficient $\nu_{\perp}=0.8$ and the tangential drag coefficient $\nu_{\|}=0.4$. The length of the curves are unity. . . . . . . . . .

9.2 Shapes of towed strings for different anisotropy of drag forces. The direction of the towing velocity is shown in the figure. The distance between the end points is 0.8 units and the length of the curve is unity. Darker curves mean higher values of $R_{\nu}$ (more isotropy in drag forces). Here $R_{\nu}=0,0.5,1$. . .

9.3 Equilibrium configurations are shown. Darker curves mean higher tangential velocity. The direction of the tangential velocity is shown in the figure. The parameters for the system are: length of the curves, $L=1$; distance between the end points, $D=0.92$; axial flow $T=0.7,0.88,0.931$; body force $q=0.71$, $0.47,0.365 \ldots \ldots \ldots \ldots \ldots \ldots \ldots$

9.4 Equilibrium configurations of curves (left) for different $\frac{L}{D}$ ratios along with their stress distributions (right) are shown in the figure. These solutions are for the special case at the transition between heteroclinic connections and one end blow up phase portraits. Here $\frac{T}{q}=2 ; \frac{L}{D}=2,4 ; m=1 \ldots . .$. . .

9.5 Stress at the trailing end of the string as a function of tangential velocity for different $\frac{L}{D}$ ratios and $m=1 \ldots \ldots \ldots \ldots$ 
A.1 Primary lobe of the heteroclinic connections along with the two fixed points are shown in the figure. . . . . . . . . . . . . . .

B.1 Orbits of heteroclinic connections in presence and absence of axial flow. . . 67

C.1 Three functions $\ldots \ldots \ldots \ldots \ldots \ldots$ 


\section{List of Tables}

8.1 Table showing the different conditions for stress behaviors at the end of the string. . . . . . . . . . . . . . . . . . 


\section{Chapter 1}

\section{Introduction and Background}

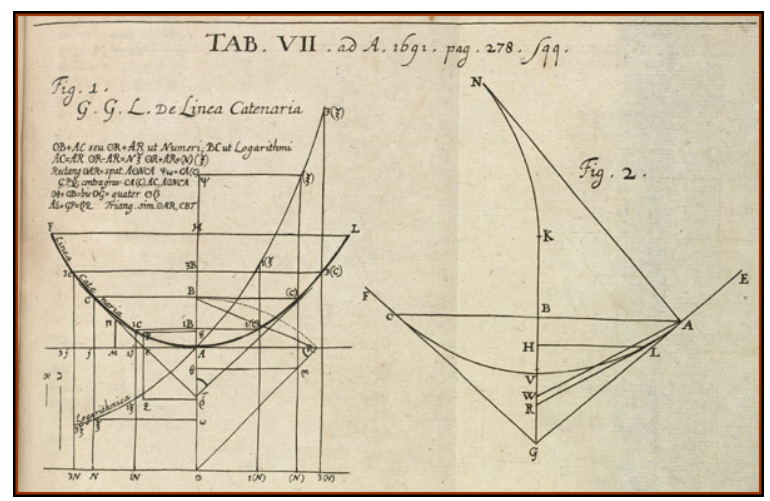

Figure 1.1: Illustration from the solutions of Leibniz and Huygen ${ }^{[1]}$. Source: http://curvebank. calstatela.edu/ catenary/catenary.htm. Used under fair use, 2015.
The catenary is the curve that a hanging chain or a string assumes due to its own weight when supported from its two ends. The mathematical properties of the catenary were first studied by Robert Hooke (1635-1703) [2] in the 1670s. In 1690, Jakob Bernoulli challenged the mathematical community to determine the exact geometry of the catenary. The problem was solved separately by Leibniz (1646-1716), Huygens (1629-1695) and Johann Bernoulli (1667-1748) [3]. All their solutions appeared in the journal Acta Eruditorum (one of the most important mathematical journals of that time) in 1691.

The word catenary derives from the latin word catena meaning chain. The English word catenary was probably first used by Thomas Jefferson [4] (third president of the United States) who wrote in a letter to Thomas Paine, on the construction of an arch for a bridge:

"I have lately received from Italy a treatise on the equilibrium of arches, by the Abbe Mascheroni. It appears to be a very scientifical work. I have not yet had time to engage in it; but I find that the conclusions of his demonstrations are, that every part of the catenary is in perfect equilibrium." [5]

Indeed the first work on the catenary by Hooke was also inspired from finding the optimal shape of an arch. The figure provided below shows the famous 'Gateway Arch' monument at St. Louis. This is an example of an inverted catenary or a catenary arch found in many 
architectures. We have also shown an example of a spider web where the small filaments attain the shape of a catenary.

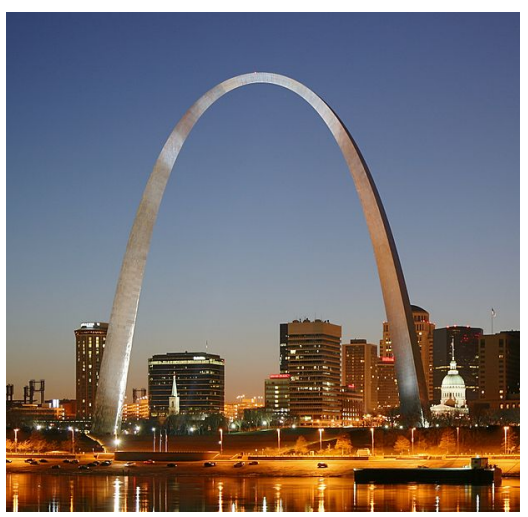

The Gateway Arch

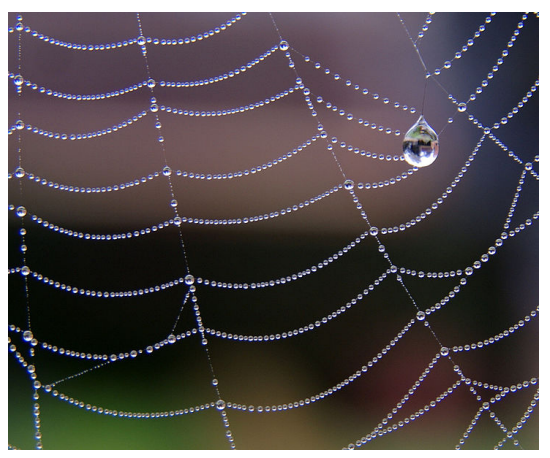

Spider web

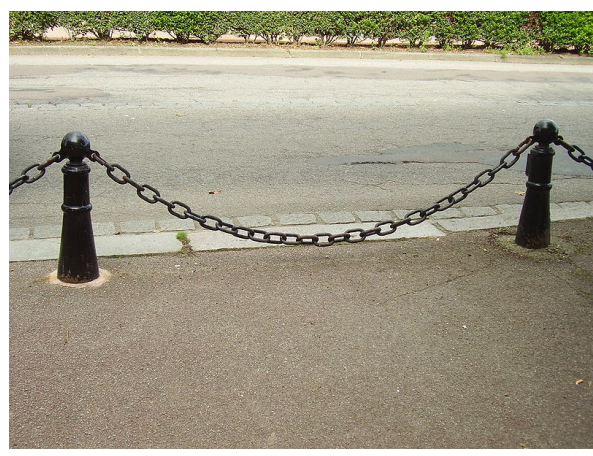

Hanging chain

Figure 1.2: Examples of the catenary arising in nature. The first image is the The Gateway Arch at St. Louis, Missouri which is an example of a catenary arch. The second image is of a spider web where the filaments have assumed the shape of a catenary. The third image is that of a hanging chain, the classic example of a catenary. Source: http://en.wikipedia. org/wiki/Catenary. Used under fair use, 2015.

It was initially presumed by Galileo that the catenary is a parabola [6]. But now it is well known that it has the graph of a hyperbolic cosine. Determining the shape of a hanging chain has now become a standard problem that can be found in textbooks on variational calculus [7]. Over the years the catenary has been considered as a static problem. However the catenary can be a solution to a dynamic problem. This dynamic nature of the catenary was probably known to Routh [8] and Lord Kelvin [9].

Our work presented here draws inspiration from the geometry of a catenary and from the works on slender bodies in fluid. Interaction of flexible slender objects with fluid flow is a challenging problem that has been studied at a large spectrum of Reynolds number with different problems in mind. At low Reynolds number one comes across the world of swimming microorganisms [10]. Locomotion at these small scales generally occurs by rotation of a corkscrew like floppy slender structure, known as flagellum [11]. Study of the interaction of flagella with the surrounding fluid was started around 50 years ago by Taylor [12], Hancock [13] and Gray [14]. Over the last two decades there has been a great number of both experimental $[15,16,17,18]$ and theoretical $[19,20,21,22,23]$ studies in this area. On the other hand, at high Reynolds number slender bodies like hanging strings, ribbons [24, 25] and flapping flags $[26,27,28]$ have drawn considerable interest. These problems have further inspired research on vortex induced vibration of slender structures ([29, 30], energy harvesting 
[31, 32]) and nature-inspired drag reduction problems [33, 34, 35]. All these problems provide a variety of rich dynamics that keeps fascinating us.

In the work discussed here we concentrate on dynamical equilibria of purely flexible, inextensible strings moving through viscous fluid with uniform velocity. There is also a flow or velocity along the axis of the string that we call the tangential velocity or the axial flow. The string is subjected to a uniform body force and local, linear, anisotropic drag forces. The solutions to the problem are generically planar. We shall provide a first order dynamical system, comprising of five parameters that gives rise to different kinds of planar curves. As a special case of our problem we recover a dynamical system representation of the catenary. It was observed by Routh [36] that in an inertial frame, adding a tangential velocity along an inextensible string does not change the shape of the equilibrium solution. However in the presence of drag forces there is a loss of symmetry and we find different equilibrium shapes. Among the many problems related to slender structures in fluid flow, our present study is relevant in the context of sedimenting filaments and towed cables. We provide a brief overview of both of these problems and how they are related to our formulation.

Studying the dynamics of filaments at low Reynolds number provides insight in the behavior of polymer molecules in complex flows and their impact on transport. There has been a number of studies $[37,38,39]$ over the last sixty years on the dynamics of such filaments in various kinds of flow. The problem of sedimentation of a filament in a viscous flow is one such problem. Dynamics of sedimenting objects in viscous fluids has been a classic problem in low Reynolds number hydrodynamics. The motion of rigid rods or filaments sedimenting in Stokes flow is well understood. At zero Reynolds number, a rigid filament while sedimenting under gravity in a viscous fluid maintains its orientation and translates at a constant velocity (Happel and Brenner [40]). The drag forces acting on the sedimenting object are anisotropic and the anisotropy depends on the geometry of the sedimenting object. Owing to this anisotropy, the filament or rod does not generally translate in the direction of the gravity. Instead it translates at a fixed angle that depends on its orientation. However, presence of inertia in the flow or any addition of flexibility to the rigid filament changes this picture of sedimentation. If there is flexibility in the filament then while sedimenting it gets reoriented with respect to the direction of the gravity. Xu and Nadim [41] studied this problem using slender body theory developed by Cox [42] and suggested that weakly flexible filaments always reorient themselves. These predictions were confirmed from numerical studies by Schlagberger and Netz [43] and Lagomarsino et al. [44]. Recent studies concerning the motion of filaments with bending resistance are numerical. (Tornberg and Shelley [45]; Llopis et al. [46], Li et al. [47]). However in the present study we have been able to obtain analytical expressions that provide us solutions for sedimentation of purely flexible filaments (with no bending resistance) in viscous flow. This problem is discussed in chapter 6 . 
In order to improve communications between the United States and Great Britain it was proposed around the 1840s and 1850s to construct a telegraph cable across the Atlantic ocean. The Atlantic Telegraph Company took up the project of laying down the transatlantic cable in 1854 and completed it in 1858. Here is a image of 1958 U.S stamp commemorating the Atlantic cable in the centenary year.

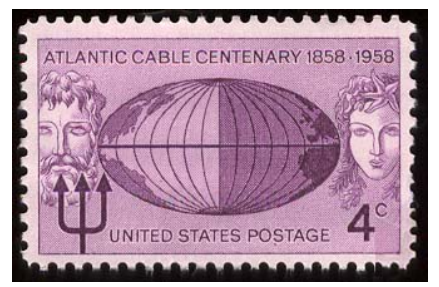

Figure 1.3: A 1958 U.S. postage stamp. Source: https://en.wikipedia.org/wiki/Transatlantic_ telegraph_cable. Used under fair use, 2015.

However the first attempted laying of the cable in the summer of 1857 ended in a disaster as the cable broke off only after laying a few hundred miles. The cable was repaired, however it broke again nearly 2 miles deep in the ocean and the operation was abandoned for a year. This sparked great interest in the study of dynamics and kinematics of laying of submarine cables. In late 1857 and early 1858, Lord Kelvin [9] and Airy [48] published their analysis of this problem. However after these initial works there has been few $[49,50,51]$ research on these problems. About a hundred years later, in 1957 Zajac [52] published his analysis on laying and recovery of submarine cables considering nonlinear drag forces. Following the work of Zajac there has been numerous theoretical and computational work on the dynamics and control $[53,54,55,56,57,58,59,60,61,62]$ of towed cables.

In the present work we have obtained equilibrium shapes for filaments that are being towed from two ends. We have also obtained solutions for equilibrium configurations of a flexible string that is being reeled out from one end and reeled in from the other. These solutions are discussed in chapter 9. We intend to extend these solutions at higher Reynolds number in future and study the problems of towing, laying and recovery of long ocean cables.

The thesis is organized as follows. We have first discussed the formulation of our problem and have shown that the generic solutions for the problem are planar. Then a first order dynamical system was obtained that depends on five parameters from which we can obtain a family of planar curves. Next, we have moved on to a discussion of the different possible geometries of the planar curves and the stress distribution along them. Later we have considered boundary value problems concerning towing of a string of fixed length. Finally, the major insights of the work have been highlighted and the possible problems that can be explored in the future are discussed. 


\section{Chapter 2}

\section{Formulation and generic solutions of the problem}

We have already discussed the nature of the problem that we are considering. In this chapter we find the equations of motion for a string from the force balance in three dimensions. We then show that the solutions to this problem are generically planar.

\subsection{Equation of motion}

Let us consider an inextensible string as shown in figure 2.1. The string is moving in a fluid with steady, uniform velocity $\overline{\mathbf{v}}$. Along with this there is a constant axial flow, $T$ (a tangential velocity) acting along the axis (or the tangent) of the string. A uniform body force $\overline{\mathbf{q}}$ and anisotropic drag forces from the surrounding fluid act upon the string. We only consider drag forces that are linear with the velocity. The shape of the string in three dimensions can be described by the use of three vectors: the tangent $\hat{\mathbf{t}}$, the normal $\hat{\mathbf{n}}$ and the binormal vector $\hat{\mathbf{b}}$. These vectors are related among themselves by the Frenet-Serret formula as:

$$
\partial_{s}\left\{\begin{array}{l}
\hat{\mathbf{t}} \\
\hat{\mathbf{n}} \\
\hat{\mathbf{b}}
\end{array}\right\}=\left[\begin{array}{ccc}
0 & \kappa & 0 \\
-\kappa & 0 & \tau \\
0 & -\tau & 0
\end{array}\right]\left\{\begin{array}{l}
\hat{\mathbf{t}} \\
\hat{\mathbf{n}} \\
\hat{\mathbf{b}}
\end{array}\right\}
$$

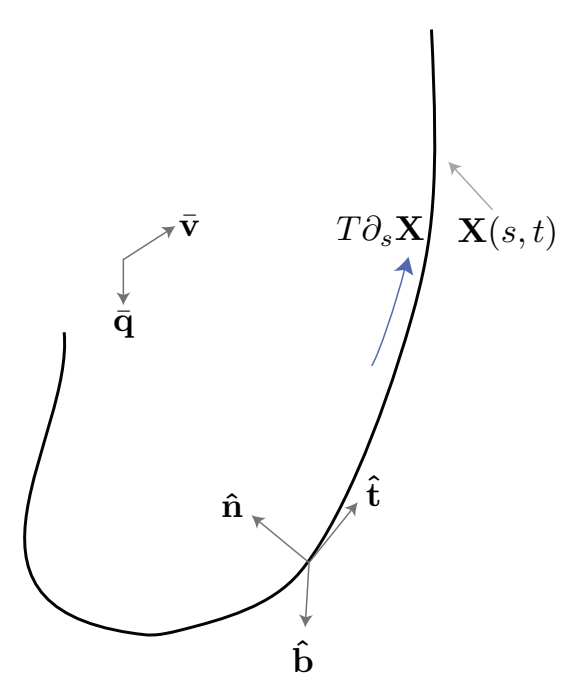

Figure 2.1: The string

where $s$ is the arc length that has been used to parametrize the curve. The position vector 
of any point along the string is denoted by $\mathbf{X}$ which is a function of the arc length and time, $t$. We know that the tangent vector for a curve is defined as:

$$
\partial_{s} \mathbf{X}=\hat{\mathbf{t}}
$$

Keeping the above relation in mind we can write:

$$
\partial_{t} \mathbf{X}=\overline{\mathbf{v}}+T \partial_{s} \mathbf{X}
$$

where $\partial_{t} \mathbf{X}$ is the Lagrangian time derivative of the position vector. Since the velocity of translation and the axial flow are constant we have:

$$
\partial_{t}^{2} \mathbf{X}=T^{2} \partial_{s}^{2} \mathbf{X}
$$

Using the above relation we write down the force balance equation for the string to obtain:

$$
m T^{2} \partial_{s}^{2} \mathbf{X}+\nu_{\|}(T+\overline{\mathbf{v}} \cdot \hat{\mathbf{t}}) \hat{\mathbf{t}}+\nu_{\perp} \overline{\mathbf{v}} \cdot(\hat{\mathbf{n}} \hat{\mathbf{n}}+\hat{\mathbf{b}} \hat{\mathbf{b}})=\partial_{s}(\sigma \hat{\mathbf{t}})+\overline{\mathbf{q}}
$$

In the left hand side of the above equation the first term is the inertia term where $m$ is the mass density of the string and it accounts for things like added mass if necessary. This term does not affect the geometry of the string however it determines the tension in the string. The next two terms represent the linear drag forces acting on the string. The dimensional drag coefficient along the tangent is $\nu_{\|}$and it is $\nu_{\perp}$ along the normal and binormal direction. If the drag coefficients are equal then the drag is isotropic. On the right hand side of the equation the first term accounts for the internal tension of the string where $\sigma$ is the stress in the filament. The second term is the uniform body force.

\subsection{Generic solutions of the problem}

We have provided the equation of motion of the filament (or string) in the previous section. In this section we discuss that the generic solutions to the problem are planar. We first project eqn (2.2) on the tangent, normal and binormal direction to obtain: 


$$
\begin{aligned}
\nu_{\|}(T+\overline{\mathbf{v}} \cdot \hat{\mathbf{t}}) & =\partial_{s} \sigma+\overline{\mathbf{q}} \cdot \hat{\mathbf{t}} \\
m T^{2} \kappa+\nu_{\perp} \overline{\mathbf{v}} \cdot \hat{\mathbf{n}} & =\sigma \kappa+\overline{\mathbf{q}} \cdot \hat{\mathbf{n}} \\
\nu_{\perp} \overline{\mathbf{v}} \cdot \hat{\mathbf{b}} & =\overline{\mathbf{q}} \cdot \hat{\mathbf{b}}
\end{aligned}
$$

Here $\kappa$ is the local curvature of the string. We have used the relation $\partial_{s}^{2} \mathbf{X}=\kappa \hat{\mathbf{n}}$ to obtain the above equations. Now we differentiate both sides of eqn (2.5) with respect to the arc length, $s$ and use the Frenet relations to obtain:

$$
\tau\left(\nu_{\perp} \overline{\mathbf{v}} \cdot \hat{\mathbf{n}}-\overline{\mathbf{q}} \cdot \hat{\mathbf{n}}\right)=0
$$

In the above expression $\tau$ is the torsion of the curve. The above expression suggests that either torsion or the bracketed quantity is zero. If torsion is not zero, then the solutions to the problem are special configurations of the string. So the generic solutions of the problem are planar with torsion being zero.

Nonzero torsion: We now show that if the torsion is nonzero then we have special solutions to the problem. If $\tau \neq 0$ then from eqn (2.6) we have,

$$
\nu_{\perp} \overline{\mathbf{v}} \cdot \hat{\mathbf{n}}=\overline{\mathbf{q}} \cdot \hat{\mathbf{n}}
$$

We can differentiate the above equation again with respect to $s$ and use eqn (2.5) to obtain,

$$
\kappa\left(\nu_{\perp} \overline{\mathbf{v}} \cdot \hat{\mathbf{t}}-\overline{\mathbf{q}} \cdot \hat{\mathbf{t}}\right)=0
$$

We can further substitute eqn (2.7) in eqn (2.4) to obtain:

$$
\kappa\left(m T^{2}-\sigma\right)=0
$$

Both of the equation (2.9) and (2.8) lead us to the solution $\kappa=0$ that is a straight line. The straight line is a special solution to the three dimensional problem. 
Arbitrary shaped curves: Now consider a case where the axial flow, $T$ is not present and there is no anisotropy in the drag forces. This means: $\nu_{\|}=\nu_{\perp}=\nu$. Under these conditions the curve can sediment with arbitrary orientation and shape in three dimensions with the tension being equal to zero. This is because the body force is balanced perfectly by the drag forces offered by the fluid. Since the drag forces are isotropic there is no effect of orientation of the curve and it can sediment with an arbitrary shape. The solution to the problem can be written as:

$$
\begin{aligned}
\mathbf{X} & \equiv \text { arbitrary } \\
T & =0 \\
\overline{\mathbf{q}} & =\nu \overline{\mathbf{v}} \\
\sigma & =0
\end{aligned}
$$

This is a special case of our problem. 


\section{Chapter 3}

\section{The planar problem and its special cases}

\subsection{Solution to the planar problem}

In the previous chapter we have shown that apart from special cases, the general solution to the problem is planar. That means we can describe the string motion by the use of tangent and normal vectors. Since the curve is planar one can also define an orthonormal coordinate system $\{\hat{\mathbf{x}}-\hat{\mathbf{z}}\}$ that spans the plane containing the curve. The $\hat{\mathbf{z}}$ vector is defined in the direction opposite to that of the body force. The force balance for the planar problem looks like:

$$
m T^{2} \partial_{s}^{2} \mathbf{X}+\nu_{\|}(T+\mathbf{v} \cdot \hat{\mathbf{t}}) \hat{\mathbf{t}}+\nu_{\perp} \mathbf{v} \cdot \hat{\mathbf{n}} \hat{\mathbf{n}}=\partial_{s}(\sigma \hat{\mathbf{t}})-q \hat{\mathbf{z}}
$$

Note that we have dropped the terms associated with the binormal direction. We have rewritten the body force as $-q \hat{\mathbf{z}}$. Since we have already incorporated the negative sign in the direction of the body force, the value of $q$ is strictly positive. 


\subsubsection{Nondimensionalization}

We shall now nondimensionalize the in plane force balance equation given by eqn (3.1). The scales used to nondimensionalize are defined as follows:

$$
\begin{aligned}
\text { Length } & \sim L \\
\text { Velocity } & \sim \sqrt{v^{2}+T^{2}+\left(\frac{q}{\nu_{\perp}}\right)^{2}} \equiv \Gamma \\
\text { Force } & \sim L^{3} \nu_{\perp} \Gamma \\
\text { Mass } & \sim \frac{L^{4} \nu_{\perp}}{\Gamma}
\end{aligned}
$$

where $L$ is some reference length scale. The dimensionless quantities denoted by tilda on the top are given by:

$$
\tilde{s}=\frac{s}{L}, \quad \tilde{T}=\frac{T}{\Gamma}, \quad \tilde{v}=\frac{v}{\Gamma}, \quad \tilde{q}=\frac{q}{\nu_{\perp} \Gamma}, \quad \tilde{m}=\frac{m \Gamma}{L \nu_{\perp}}
$$

We substitute the dimensionless quantities in eqn (3.1) and subsequently drop the tilda for the sake of convenience and get:

$$
m T^{2} \partial_{s}^{2} \mathbf{X}+R_{\nu}(T+\mathbf{v} \cdot \hat{\mathbf{t}}) \hat{\mathbf{t}}+\mathbf{v} \cdot \hat{\mathbf{n}} \hat{\mathbf{n}}=\partial_{s}(\sigma \hat{\mathbf{t}})-q \hat{\mathbf{z}}
$$

In this equation all the quantities are dimensionless. There is a new parameter in the system $R_{\nu}=\frac{\nu_{\|}}{\nu_{\perp}}$. This is the ratio of the drag coefficients along the tangent and the normal direction. One can say that $R_{\nu}$ gives a measure of anisotropy of drag forces in the problem. If $R_{\nu}$ is unity then the drag forces are isotropic. For a slender body in Stokes flow one can show ([63], [42]) that $R_{\nu}=0.5$.

Because of our choice of the velocity scale $\Gamma$, the normalized (or dimensionless) quantities from (3.2) satisfy the following relation:

$$
T^{2}+|\mathbf{v}|^{2}+q^{2}=1
$$

These normalized quantities lie on the 2 -sphere $\left(\mathbb{S}^{2}\right)$. We shall use this fact in the later sections for a better understanding of the problem. 


\subsubsection{Obtaining a first order dynamical system}

Since the string is planar, it can be described by the use of a single variable, the angle between the horizontal, $\hat{\mathbf{x}}$ and the tangent, $\hat{\mathbf{t}}$, This is called the tangential angle $(\theta)$ and is also known as the Whewell angle.

The angle is shown in figure 3.1 and it is a function of the arc length $s$. Figure 3.1 shows another angle $\phi$ that is the angle between the velocity vector $\mathbf{v}$ and the horizontal. We shall use the angle $\phi$ in later chapters to define the direction of the velocity of translation. One can relate the tangent and normal vectors to the orthonormal basis using the rotation matrix:

$$
\left\{\begin{array}{c}
\hat{\mathbf{t}} \\
\hat{\mathbf{n}}
\end{array}\right\}=\left[\begin{array}{cc}
\cos \theta & \sin \theta \\
-\sin \theta & \cos \theta
\end{array}\right]\left\{\begin{array}{l}
\hat{\mathbf{x}} \\
\hat{\mathbf{z}}
\end{array}\right\}
$$

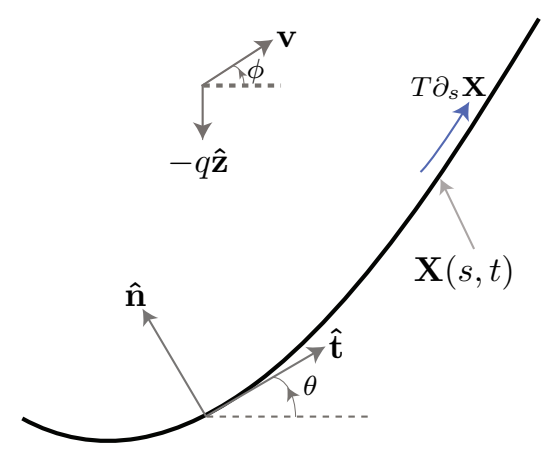

Figure 3.1: Planar curve

Using the Frenet formula provided in eqn (2.1) and the above relations it follows that the arc length derivative of the tangential angle $\theta$ is the local curvature of the string; that is: $\partial_{s} \theta=\kappa$. We can project the force balance equation (3.3) on the tangent and the normal directions and use the relation (3.5) to obtain:

$$
\begin{aligned}
\partial_{s} \sigma & =R_{\nu}(T+\mathbf{v} \cdot \hat{\mathbf{x}} \cos \theta+\mathbf{v} \cdot \hat{\mathbf{z}} \sin \theta)+q \sin \theta \\
\sigma \partial_{s} \theta & =m T^{2} \partial_{s} \theta+(-\mathbf{v} \cdot \hat{\mathbf{x}} \sin \theta+\mathbf{v} \cdot \hat{\mathbf{z}} \cos \theta)+q \cos \theta .
\end{aligned}
$$

From the above two equations we can eliminate $\sigma$ and obtain,

$$
\partial_{s}\left[\frac{(-\mathbf{v} \cdot \hat{\mathbf{x}} \sin \theta+\mathbf{v} \cdot \hat{\mathbf{z}} \cos \theta)+q \cos \theta}{\partial_{s} \theta}\right]=R_{\nu}(T+\mathbf{v} \cdot \hat{\mathbf{x}} \cos \theta+\mathbf{v} \cdot \hat{\mathbf{z}} \sin \theta)+q \sin \theta .
$$

Now we multiply by the inverse of the bracketed quantity on the left to obtain,

$$
\partial_{s} \ln \left[\frac{(-\mathbf{v} \cdot \hat{\mathbf{x}} \sin \theta+\mathbf{v} \cdot \hat{\mathbf{z}} \cos \theta)+q \cos \theta}{\partial_{s} \theta}\right]=\left[\frac{R_{\nu}(T+\mathbf{v} \cdot \hat{\mathbf{x}} \cos \theta+\mathbf{v} \cdot \hat{\mathbf{z}} \sin \theta)+q \sin \theta}{(-\mathbf{v} \cdot \hat{\mathbf{x}} \sin \theta+\mathbf{v} \cdot \hat{\mathbf{z}} \cos \theta)+q \cos \theta}\right] \partial_{s} \theta
$$


We should note that the right hand side (RHS) of the above equation is a total derivative. It is now useful to introduce the following notations (or rescaling) in order to facilitate the representation of the result of integration. Let us define the new quantities as follows,

$$
\begin{aligned}
\Delta & =\sqrt{(\mathbf{v} \cdot \hat{\mathbf{x}})^{2}+(\mathbf{v} \cdot \hat{\mathbf{z}}+q)^{2}} \\
\bar{T} & =\frac{R_{\nu} T}{\Delta} \\
\bar{U} & =\frac{\mathbf{v} \cdot \hat{\mathbf{x}}}{\Delta} \\
\bar{W} & =\frac{\mathbf{v} \cdot \hat{\mathbf{z}}}{\Delta} \\
\bar{Q} & =\frac{q}{\Delta}
\end{aligned}
$$

Introducing the above rescaling, the result of the integration of eqn (3.9) is,

$$
\begin{aligned}
\ln \left|\left[\frac{-\bar{U} \sin \theta+(\bar{W}+\bar{Q}) \cos \theta}{\partial_{s} \theta}\right]\right| & =\tilde{C}+\bar{T} \ln \left|\left[\frac{1+\bar{U}+(\bar{W}+\bar{Q}) \tan \frac{\theta}{2}}{1-\bar{U}-(\bar{W}+\bar{Q}) \tan \frac{\theta}{2}}\right]\right| \\
& -\left[R_{\nu} \bar{U}^{2}+\left(R_{\nu} \bar{W}+\bar{Q}\right)(\bar{W}+\bar{Q})\right] \ln |[-\bar{U} \sin \theta+(\bar{W}+\bar{Q}) \cos \theta]| \\
+ & +\bar{U}\left(R_{\nu}-1\right) \bar{Q} \theta
\end{aligned}
$$

where a term proportional to $\ln \Delta$ has been absorbed into the constant of integration $\tilde{C}$. Absolute values are introduced in the above expression because the trigonometric terms in the parentheses can be negative for some choices of the parameters. Since there is no meaning of natural logarithm of a negative number for a physical system we introduce these absolute values. After some simple algebraic manipulation we obtain our first order dynamical system:

$\partial_{s} \theta=C e^{\bar{U}\left(1-R_{\nu}\right) \bar{Q} \theta}\left|\left[\frac{1-\bar{U}-(\bar{W}+\bar{Q}) \tan \frac{\theta}{2}}{1+\bar{U}+(\bar{W}+\bar{Q}) \tan \frac{\theta}{2}}\right]\right|^{\bar{T}}[|-\bar{U} \sin \theta+(\bar{W}+\bar{Q}) \cos \theta|]^{\left[1+R_{\nu} \bar{U}^{2}+\left(R_{\nu} \bar{W}+\bar{Q}\right)(\bar{W}+\bar{Q})\right]}$

Now let us look at the parameters that affect the first order system and govern the geometry of the string. From (3.15) it may seem that there are six parameters for the system:

- $R_{\nu}$ : The ratio between the drag coefficients in the tangential and normal directions 
that provides the measure of anisotropy of the drag forces in the flow. For our problem we shall be looking at the range where $R_{\nu} \in[0,1]$.

- $\bar{U}$ : The velocity of translation in the horizontal $(\hat{\mathbf{x}})$ direction.

- $\bar{W}$ : The velocity of translation in the vertical ( $\hat{\mathbf{z}})$ direction.

- $\bar{Q}$ : The body force acting in the $-\hat{\mathbf{z}}$ direction.

- $\bar{T}$ : The tangential/axial flow.

- $C$ : A scaling constant.

However not all of these parameters are independent. The dimensionless velocity, body force and axial flow lie on the 2-sphere and are related by the relation (3.4). Because of this relation we have a first order dynamical system that is governed by five independent parameters.

For the ND variables we are interested in the range where $T \in[-1,1]$. Since $q$ and $|\mathbf{v}|$ are strictly positive, these two parameters will be in a range of $[0,1]$. The angle of translation $\phi$ made by the velocity with the positive $\hat{\mathbf{x}}$ direction will be varying between $\left[-\frac{\pi}{2}, \frac{\pi}{2}\right]$.

\subsection{Representations of the dynamical system}

In the previous section we have obtained our first order dynamical system given by eqn (3.15). We can represent our first order dynamical system using phase portraits. The curves can then be obtained by integrating along phase trajectories. Because of the five parameters in the system, the phase space is very rich. For different choices of the parameters one can obtain different kinds of phase portraits. In this section we discuss the possible behaviors of the phase portraits.

\subsubsection{Fixed Points, scaling and symmetries}

In our parameter space for certain choices of the parameters one can obtain heteroclinic connections as a phase portrait. The figure 3.2 shows such a phase portrait. Heteroclinic orbits are obtained between two fixed points that are separated by an angle $\pi$. A set of heteroclinic orbits with different scaling constants constitute a lobe. Here we have shown 
three lobes that go over an angle of $3 \pi$. There are infinitely many lobes that we have not shown in the figure.

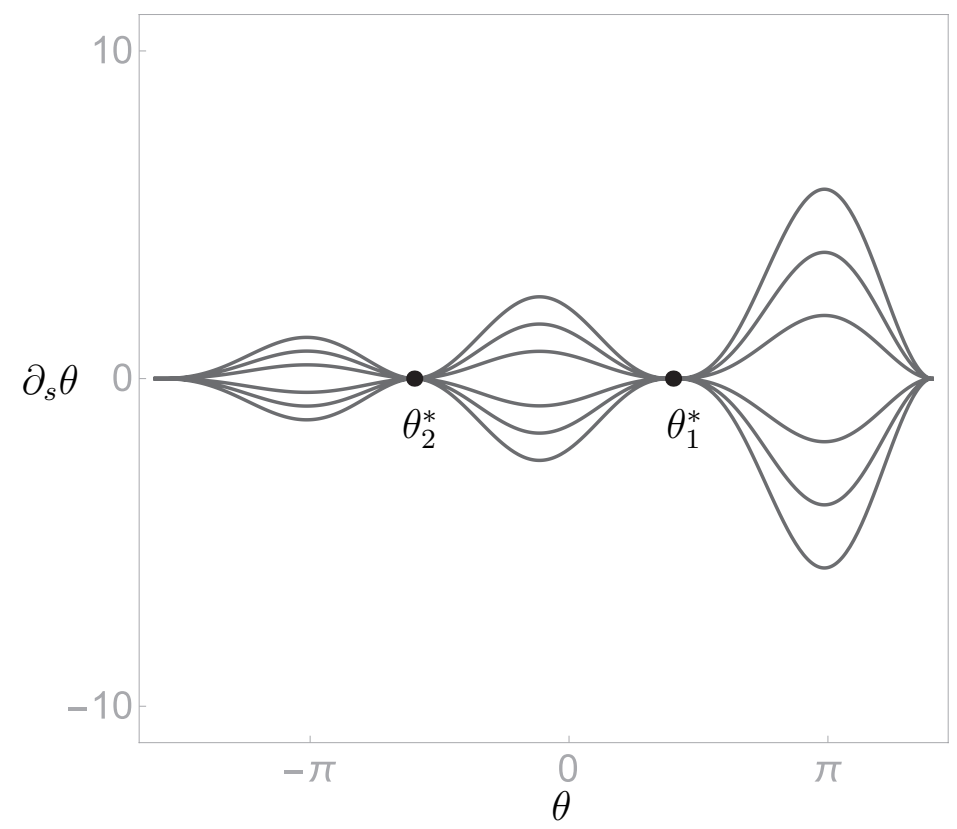

Figure 3.2: Heteroclinic connections as phase portrait. The figure shows three lobes of the phase portrait that go over an angle of $3 \pi$. Two fixed points have been marked on the figure.

We note from the above figure that there are infinitely many fixed points in our dynamical system. These fixed points are separated by an angle of $\pi$ between them. We have labeled two of the fixed points in the phase portrait:

$$
\begin{aligned}
& \theta_{1}^{*}=\tan ^{-1}\left(\frac{\bar{W}+\bar{Q}}{\bar{U}}\right) \\
& \theta_{2}^{*}=\theta_{1}^{*}-\pi
\end{aligned}
$$

The process for obtaining these fixed points is outlined in appendix A. The lobe between these two fixed points is called the primary lobe.

We shall also like to point out that the phase trajectories on the lower half of the plane are just reflections of those in the upper half. Curves obtained from the lower half of the phase portrait are rotations by $\pi$ of the curves obtained from the upper half of the plane. This means that reflection about the horizontal plane in the phase space corresponds to 
rotation in the plane of the actual curves. In this section we want to show that all the phase trajectories from different lobes can be thought to be equivalent if appropriate scaling and reflection are used.

Figure 3.3 shows three lobes of heteroclinic connection in the upper half of the plane. We have used different colors to indicate the direction of the axial flow in the curve. Let us consider the red colored phase trajectory for negative axial flow in the primary lobe. We observe that if this is reflected about a vertical axis passing through $\theta_{1}^{*}$ and then scaled, one can obtain the blue colored phase trajectory for positive axial flow. This happens because of the form of the trigonometric function with which the axial velocity is associated. Note that moving to an adjacent lobe of the primary lobe means moving through an angle of $\pi$ in the phase space. This causes the curve to rotate through an angle of $\pi$. Since we want similar curves we need to do a rotation of the integrated curve. This corresponds to a reflection of the orbits in the phase space about the horizontal plane. This is why we need to change the sign of the scaling constant.

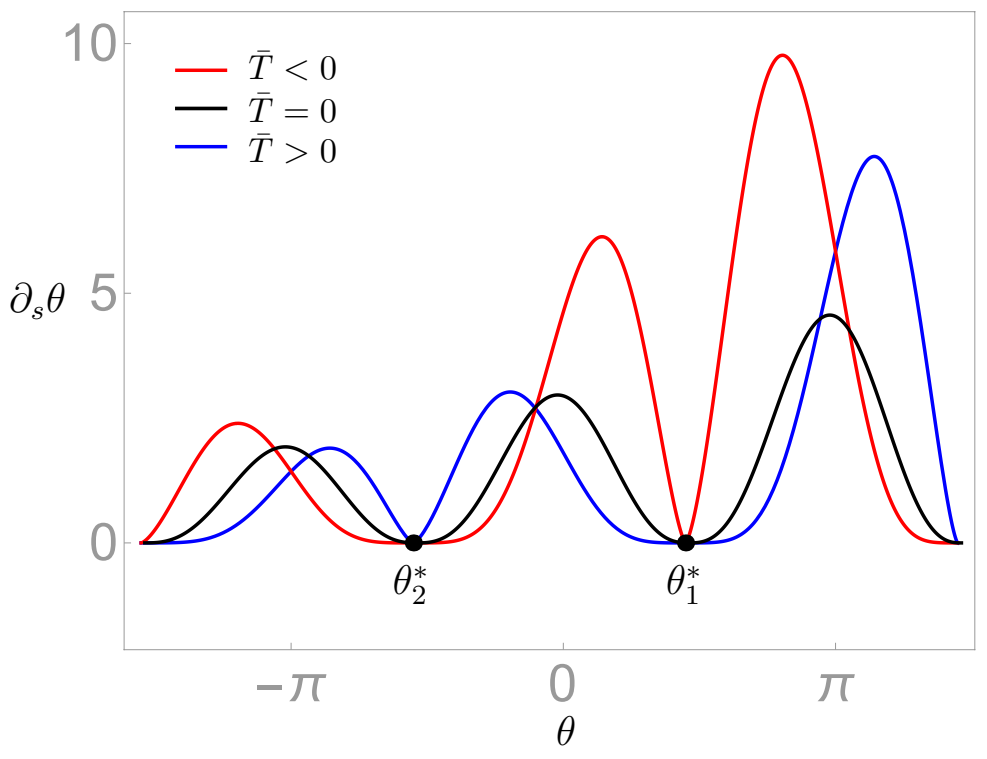

Figure 3.3: Scaling and asymmetry in axial flow

Our discussion so far can be written in the following way:

$$
C \partial_{s} \theta\left(\theta_{2}^{*}+\theta,-\bar{T}\right) \equiv-C\left(e^{-\bar{Q} \bar{U}\left(1-R_{\nu}\right) \pi}\left|\frac{\bar{U}+1}{\bar{U}-1}\right|^{\bar{T}}\right) \partial_{s} \theta\left(\theta_{1}^{*}+\theta,+\bar{T}\right)
$$

The derivation for the above relation is provided in appendix B. 
The discussion provided here implies that we can always work with the primary lobe that is located between $\theta_{1}^{*}$ and $\theta_{2}^{*}$. Curves obtained from any other lobe on the phase portrait can be shown equivalent to that obtained from the primary lobe by the use of appropriate scaling and reflection operation. All the curves that we discuss here in the following chapters are obtained from this primary lobe.

\subsubsection{Different phase portraits}

We have so far discussed heteroclinic connections in our phase space. However there are other kinds of phase trajectories that may arise depending on the choice of our parameters. Apart from the heteroclinic connections one can have two end blow up and one end blow up phase portraits where the orbits of the phase portrait go to infinity at the poles. Figure 3.4 shows all these three kinds of phase portraits for our dynamical system.

These different kinds of phase portraits are observed depending on the sign of the exponents of our dynamical system. For convenience let us define the following:

$$
k=1+R_{\nu} \bar{U}^{2}+\left(R_{\nu} \bar{W}+\bar{Q}\right)(\bar{W}+\bar{Q})
$$

Depending on the relative magnitudes of $k$ and $\bar{T}$ we can have three distinct behaviors. We do not outline the derivation of the conditions for these behaviors in this section. The detailed derivation can be found in appendix $\mathrm{C}$.

Heteroclinic connections: In our parameter space, if we choose our parameters such that $k+\bar{T}>0$ and $k-\bar{T}>0$ then we have heteroclinic connections. The first diagram of figure 3.4 shows this kind of phase portrait.

Two end blow up: There can be a region in parameter space where both $k+\bar{T}<0$ and $k-\bar{T}<0$. For these choices of the parameters we observe two end blow up phase portraits. For this case, the phase trajectories go to infinity (or blows up) at $\theta_{1}^{*}$ and $\theta_{2}^{*}$. Since the curvature blows up to infinity at these points, they are now called poles instead of fixed points. This phase portrait is shown in the middle diagram of figure 3.4. The singularity in the curvature is integrable and there are no singularities in the tangential angle of the curve. The curves obtained upon further integration are thus well behaved. 


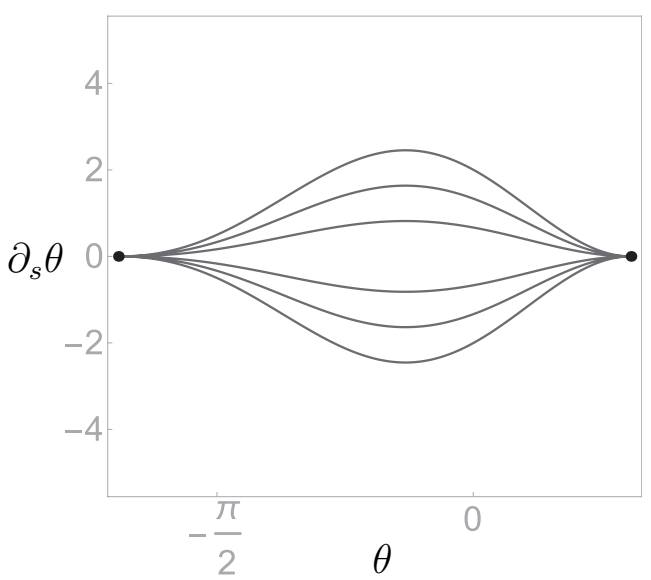

Heteroclinic connection

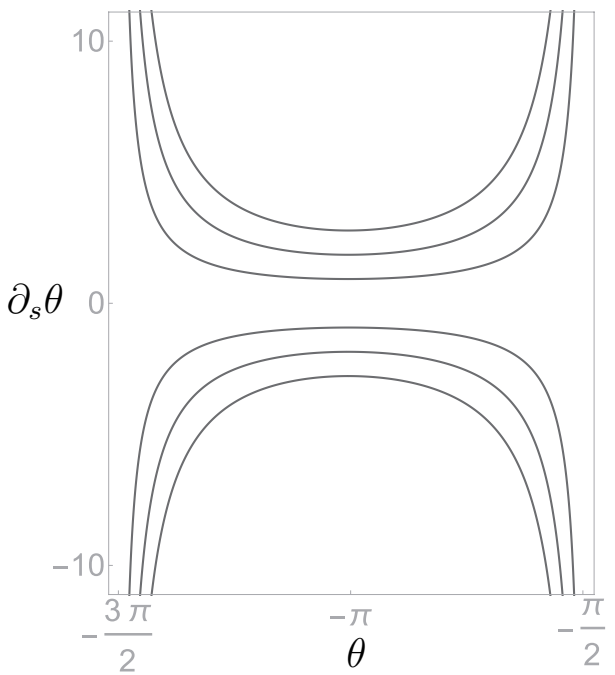

Two end blow up

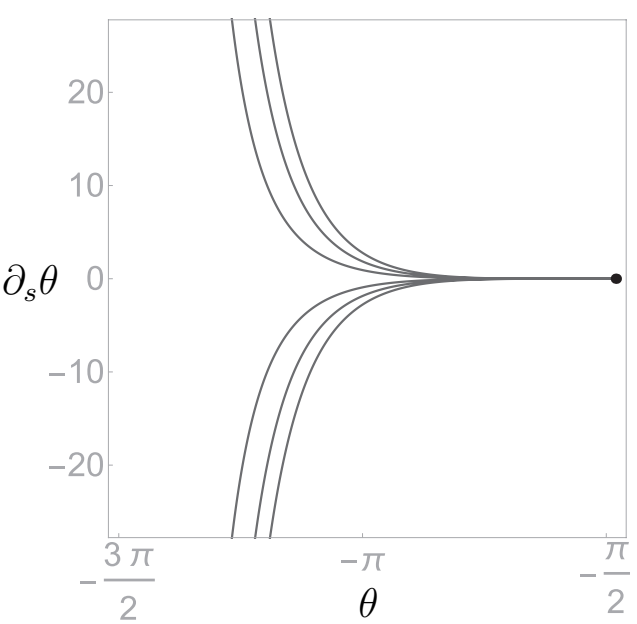

One end blow up

Figure 3.4: This figure shows three possible kinds of phase portraits that one can observe for different choices of parameters.

One end blow up: The last possible case is the one end blow up. If either $k+\bar{T}<0$ or $k-\bar{T}<0$ then one can have blow up at one of the fixed points of the phase portrait. So we shall have one pole and one fixed point in the phase portrait. The curvature blows up at the pole and goes to zero at the fixed point. This has been shown in the last diagram from figure 3.4 .

Special cases: In the parameter space one encounters some special cases when there is a transition between any of these three different phase portraits discussed above. We shall discuss the origin and the behavior of these special cases in section 4.1. 


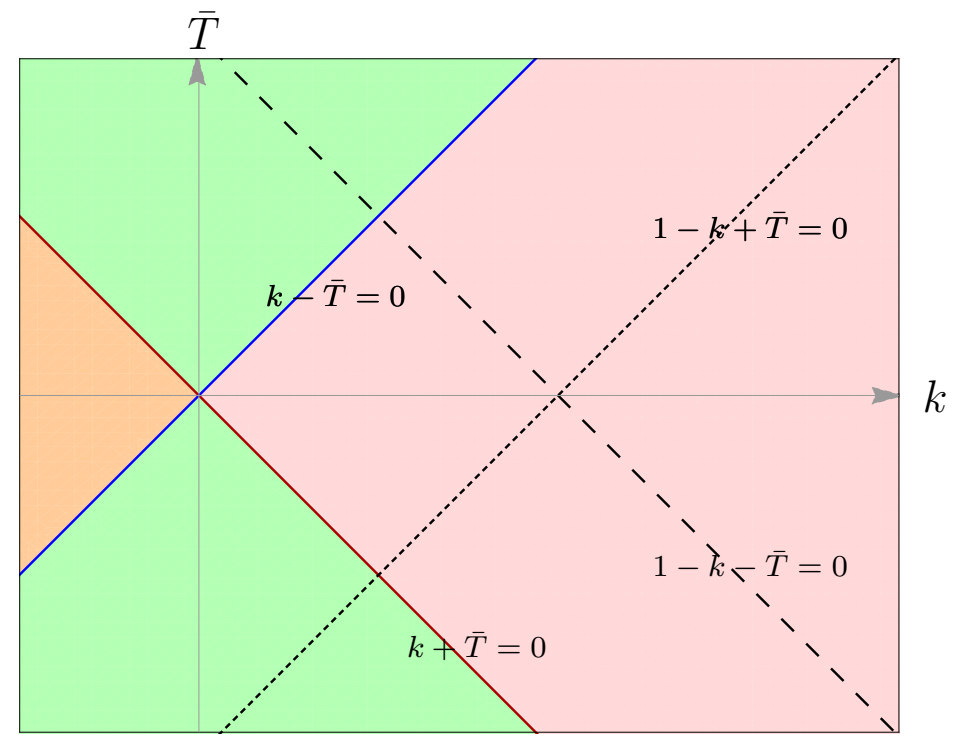

Figure 3.5: In the above figure, pink region corresponds to heteroclinic connections, the green region corresponds to one end blow up and the orange colored region corresponds to two end blow up.

We can make a simple graphical representation in order to summarize the different behaviors that one encounters in the dynamical system. Figure 3.5 represents a plot on the $k-\bar{T}$ plane. The lines drawn in blue and brown separate different regions. The special cases that we mentioned occur on these lines whenever there is a transition from one kind of behavior to another. We have also drawn two dashed lines on this figure. These lines correspond to different behaviors of the stress at the end of the string. We shall provide a detailed discussion on these behaviors in chapter 8 .

\subsubsection{Characteristics of curves arising from different phase trajec- tories}

So far the different types of phase portraits have been classified. Here we discuss the fundamental difference between the curves that are obtained upon integrating along a phase trajectory for different phase portraits.

A curve is obtained by integrating along a phase trajectory. As expected, the curves obtained from three different kinds of phase portraits differ in their geometries. We will discuss their geometry in the upcoming chapters. Here we shall like to point out the most important differences between the curves obtained from different phase trajectories. The fundamental 
difference lies in the length of the curves. The length of a curve obtained upon integration between two fixed points (or poles) is given by the following expression:

$$
L=\int_{\theta_{2}^{*}}^{\theta_{1}^{*}} \frac{d \theta}{\partial_{s} \theta}
$$

For a heteroclinic connection the curvature is zero at the two fixed points and hence the integral is improper. As a result, the length of the curve obtained is infinite. However for the two end blow up case the integral for the length is well behaved and the length of the curve is finite. Following the same argument we can conclude that a curve obtained from one end blow up is semi-infinite. We shall outline the numerical procedure for obtaining curves in the following subsection.

\subsubsection{Numerical method for obtaining curves}

We noted that the curves obtained from the heteroclinic connections are of infinite length. Because of this reason it is not possible to integrate between the two fixed points of a heteroclinic connection. In order to avoid this difficulty we integrate between $\theta_{2}^{*}+\epsilon$ and $\theta_{1}^{*}-\epsilon$ where $\epsilon$ is some small cut off constant.

We are interested to compare the geometry of the curves obtained from different orbits. The comparison is only meaningful when all the curves have the same length. In order to do this we choose an appropriate scaling constant $C$ such that the curves obtained upon integration are all of unit length. The parameter $C$ is obtained by performing the numerical integration:

$$
C=\int_{\theta_{2}^{*}+\epsilon}^{\theta_{1}^{*}-\epsilon} \frac{d \theta}{\partial_{s} \theta}
$$

After we have obtained the scaling constant we integrate the following three ODEs to obtain a curve:

$$
\begin{aligned}
& \partial_{s} \theta=C \mathcal{P}(\theta) \\
& \partial_{s} x=\cos \theta \\
& \partial_{s} z=\sin \theta
\end{aligned}
$$


where $\mathcal{P}$ is the dynamical system given by (3.15). The integration is done from $s=0$ to $s=1$ since the length of the curve is unity. The initial conditions that are used for this integration are given by:

$$
\theta(0)=\theta_{2}^{*}+\epsilon, \quad x(0)=x_{0}, \quad z(0)=z_{0}
$$

The choice of origin (initial conditions for $x$ and $z$ ) is arbitrary since this does not affect the geometry of the curve. It just shifts the curve in space. Note that since we are using a cut off, the curves do not exactly subtend an angle of $\pi$ between the two ends. We are actually integrating over an angle of $\pi-2 \epsilon$.

\subsection{Special cases of the problem}

\subsubsection{Pure axial flow}

This is an interesting problem. Let us think about two reels of string or chain on a horizontal plane. The string is being reeled out from one spool and is reeled into the other at an uniform rate. This means there is a constant axial flow (or tangential velocity) along the string. There is also drag force acting along the tangent of the string. There is no role of the normal drag since there is no velocity associated with the normal direction. The body force in this problem is zero.

Now we want to figure out the equilibrium shape of the string. Let us write the force balance equation for this problem:

$$
m T^{2} \partial_{s}^{2} \mathbf{X}+\nu_{\|} T \hat{\mathbf{t}}=\partial_{s}(\sigma \hat{\mathbf{t}})
$$

If we project this force balance equation on the tangent and normal direction we get:

$$
\begin{aligned}
\left(\sigma-m T^{2}\right) \partial_{s} \theta & =0 \\
\partial_{s} \sigma & =\nu_{\|} T
\end{aligned}
$$

From (3.23) we get a solution for the stress as:

$$
\sigma=\nu_{\|} T s+\sigma_{i n}
$$


However from eqn (3.22) we get:

$$
\sigma=m T^{2} \text { or } \partial_{s} \theta=0
$$

This clearly shows that the stress distribution one obtains from (3.23) contradicts that obtained from (3.22). The only meaningful solution is the one obtained by setting $\partial_{s} \theta$ to zero. As a result, a straight line will be obtained as a solution that has linearly varying stress along the arc length.

Our above discussion shows that there are no equilibrium solutions for pure axial flow with drag forces other than the special solution of a straight line. However if the body force was nonzero then there would have been other equilibrium solutions. This will be discussed later in chapter 9.

\subsubsection{Straight line solutions from fixed points}

In chapter 2, we have already discussed how straight line solutions constitute a special case of the problem. These solutions arise from the fixed points of the dynamical system. For a dynamical system, a fixed point is a point where the vector field identically vanishes. Because

of this if one starts with fixed points as initial conditions, the dynamical system will remain there. This is why the fixed points are themselves a solution to the problem.

The straight line solutions arise from the fixed points: $\theta_{1}^{*}, \theta_{2}^{*}$. For these points we shall have:

$$
\begin{gathered}
\partial_{s} \theta=0 \\
\Longrightarrow \theta=\theta_{1}^{*} \text { or } \theta_{2}^{*}
\end{gathered}
$$

The straight lines arising from these fixed points will have a slope equal to $\tan \theta^{*}$. It is important to note that since tan has a periodicity of $\pi$ the straight line solutions resulting from the two fixed points will have the same slope. 


\section{Chapter 4}

\section{Parameter space visualization}

It has already been pointed out that we are dealing with a problem with five parameters. As we pick different values of parameters we see a number of different behaviors in the phase portrait that provide us different kinds of curves. It turns out that there is a nice geometric way to think about the parameter space and understand the different kinds of phase portrait and the transition between them. In this chapter we provide the geometric structure of the parameter space and suggest how to approach this problem.

\subsection{Octant of a sphere and its projection}

We have already pointed out the conditions for different behaviors of the phase portrait in section 3.2. The different behaviors depend only on the exponents $k$ and $\bar{T}$. As previously mentioned, a detailed derivation of these conditions can be found in appendix C.

We have a five parameter system. In order to understand the effect of these parameters we first fix a few of them. Let us fix the anisotropy in the drag forces $\left(R_{\nu}\right)$ and the angle of translation $(\phi)$ to some fixed values. The scaling parameter $C$ does not affect the nature of the different phase portraits. So essentially we have three parameters now. They are the body force, magnitude of the velocity of translation and the axial flow (or the tangential velocity). They are related by eqn (3.4). Instead of looking at the rescaled quantities, it will be easier to think in terms of our nondimensional parameters: $q,|\mathbf{v}|$ and $T$. In section 3.1 we have already shown that the nondimensionalized quantities are related by :

$$
q^{2}+|\mathbf{v}|^{2}+T^{2}=1
$$


We know from this relation that these quantities lie on the 2 -sphere in $q,|\mathbf{v}|, T$ space. Now the magnitude of velocity and the body force are both strictly positive. The sign of the tangential velocity affects the shape of a curve. However, it does not affect the transition between different kinds of phase portraits. This can be appreciated from figure 3.5. In that figure we see that the regions for different phase portraits have the same shape and structure for both positive and negative $\bar{T}$. In fact, the lower half of the plane in figure 3.5 is reflection of the upper half. Because of this, it is sufficient to look at only positive values of the axial flow in order to understand the geometry of the parameter space that gives rise to different phase portraits. So we are going to study only the positive octant of the unit sphere

Upon the use of the inequalities provided in the appendix $\mathrm{C}$, we obtain the following structure of the octant of our sphere as shown in figure 4.1.

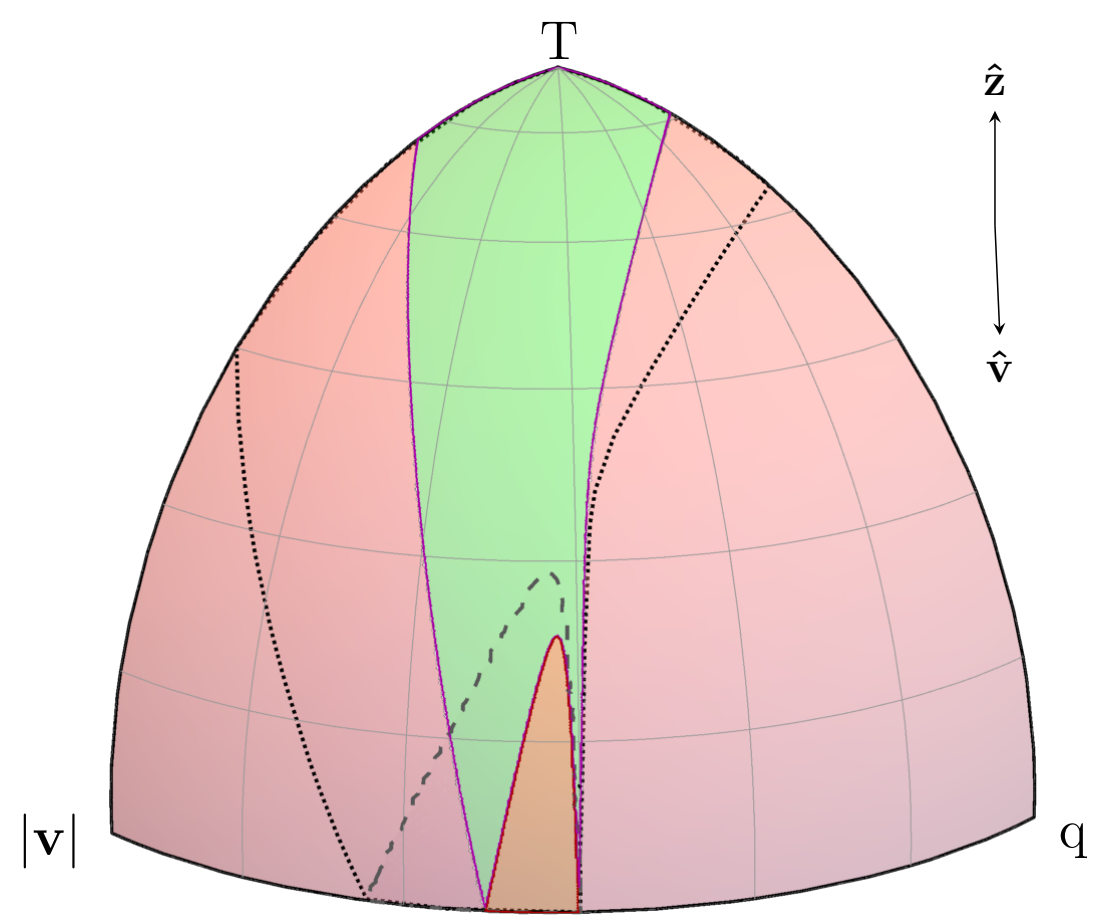

Figure 4.1: Different regions on the sphere for Stokes flow with angle of translation of the string being $-88^{\circ}$. Pink: Heteroclinic connections, Orange: Two end blow up, Green: One end blow up .

However this octant representation is not very easy to visualize. So we use orthographic projection of this octant onto the $|\mathbf{v}|-q$ plane that is more easy to comprehend. This projected view is shown in figure 4.2 . 


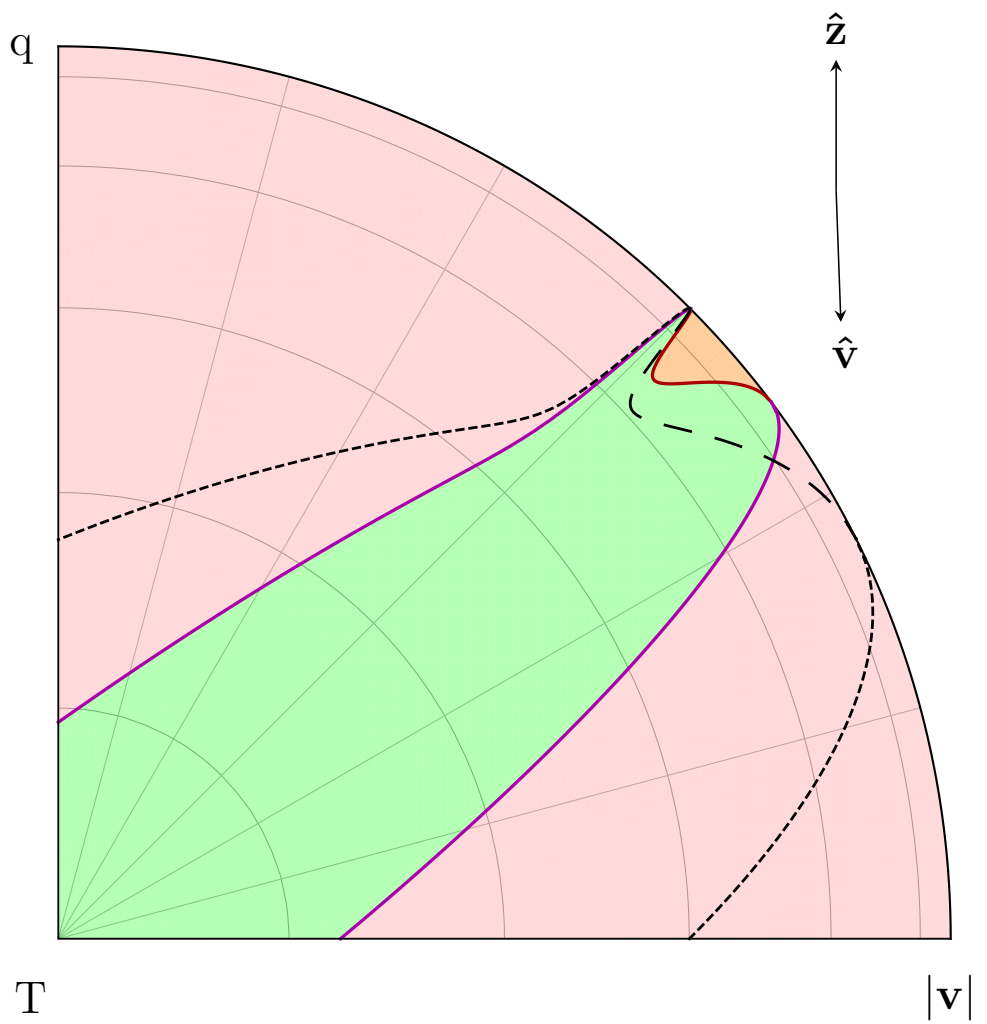

Figure 4.2: Projected view of the octant of the sphere on the $|\mathbf{v}|-q$ plane. This projection is for Stokes flow $\left(R_{\nu}=0.5\right)$ and the angle of translation is $-88^{\circ}$.

Both the sphere and the projected views show boundaries of different colors that separate the qualitatively different regions of phase portraits. These boundaries correspond to special cases in the phase space that one encounters while transitioning from one behavior to another. The dashed lines seen on the two figure correspond to qualitatively different behavior of the stress at the end of the string. We shall discuss this behavior in chapter 8 .

There are several ways to move through this parameter space. One can fix the axial flow and move along one of the arcs (or latitude lines) shown in the projection (or on the sphere). This cut through phase space will help us to understand the effect of the velocity of translation at a fixed value of the axial flow.

Another meaningful way to move in the parameter space is to move along the lines of constant $\frac{q}{|\mathbf{v}|}$. These are the radial lines drawn in the figure 4.2. Moving along these lines will help us to understand the effect of axial flow. 

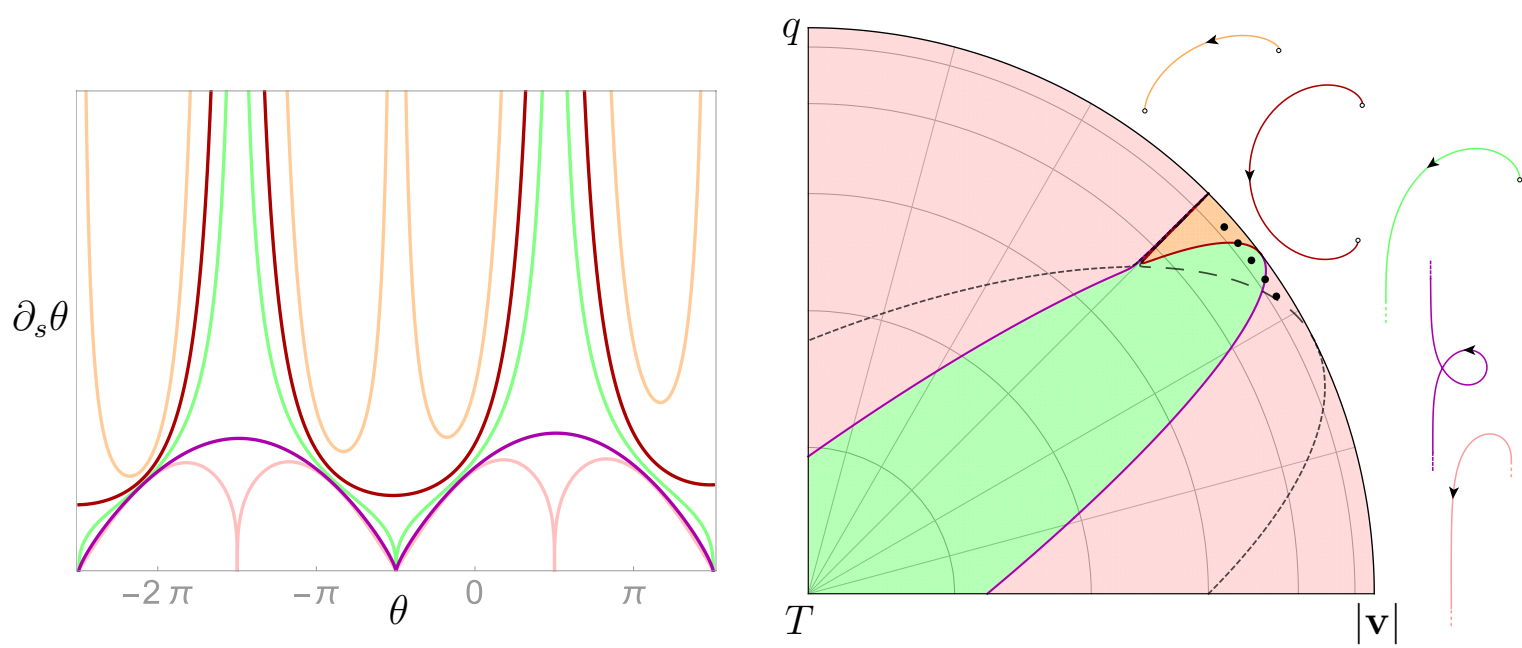

Figure 4.3: Phase trajectories (left) and configurations (right) corresponding to the dotted locations in the central diagram. The translational velocity is nearly collinear with the body force $q$, with the angle of translation being $-89.9^{\circ}$. There is an additional tangential velocity $T=0.2$ in the arrowed directions and the anisotropy $R_{\nu}=0.5$ (Stokes flow). Dashed ends indicate that the configuration extends indefinitely, while a dot on the end of a curve indicates that the configuration terminates.

Figure 4.3 shows a cut through the parameter space. Here we move along one of the iso- $\mathrm{T}$ lines. The figure also provides the curves and different phase orbits. We observe that $\pi$ periodic phase trajectories are found in the three colored regions, and $2 \pi$ periodic phase trajectories on the boundaries corresponding to the special cases. The scaling used for the curves are not same as the scaling used to represent the orbits. In order to represent the orbits in a non intersecting way we have used the following scaling $C=4 \bar{Q} \bar{U}\left(1-R_{\nu}\right) \pi$. We shall discuss these curves separately in the later chapters with more details.

\subsection{Effect of changing the angle of translation $\phi$}

In our five parameter family the velocity $\bar{U}$ and $\bar{W}$ are affected by the angle of translation of the filament or string. As we change the angle of translation, the geometry of the different regions in the parameter space also changes. In figure 4.2 the different regions have been shown for almost downward translation of the string. This is because that this translation angle captures all possible behaviors of the string. We shall now see in figure 4.4 that if the angle of translation changes, some of the phase portraits are not accessible in the parameter space. We show this variation for the case of Stokes flow. 


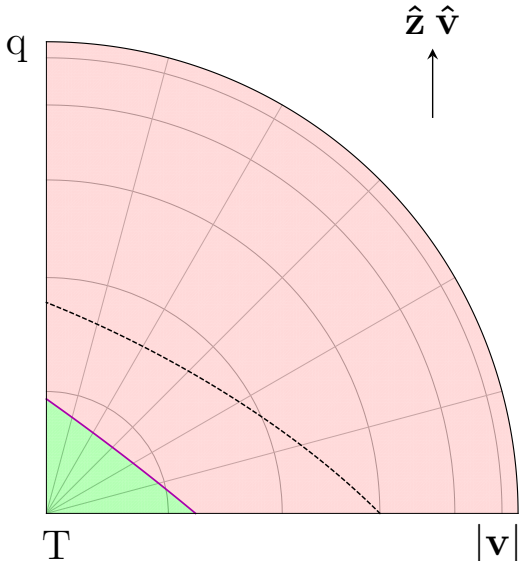

(a) $\phi=90^{\circ}$

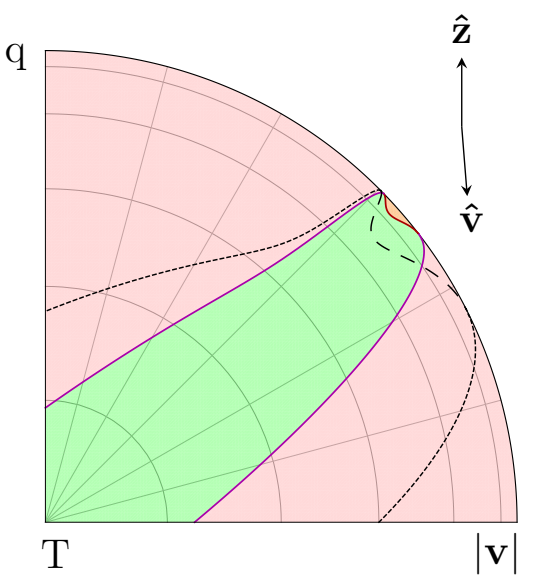

(d) $\phi=-85^{\circ}$

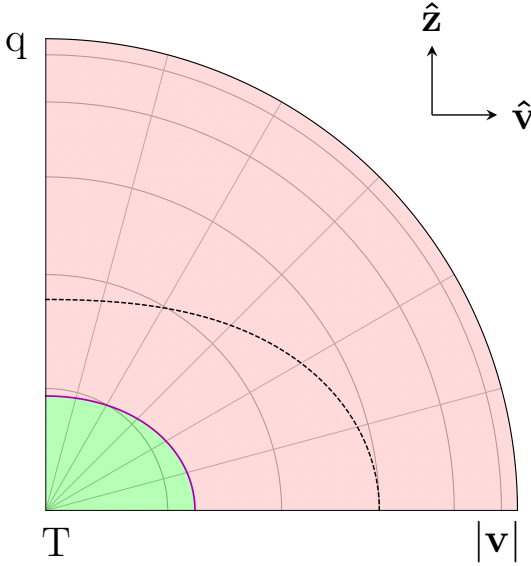

(b) $\phi=0^{\circ}$

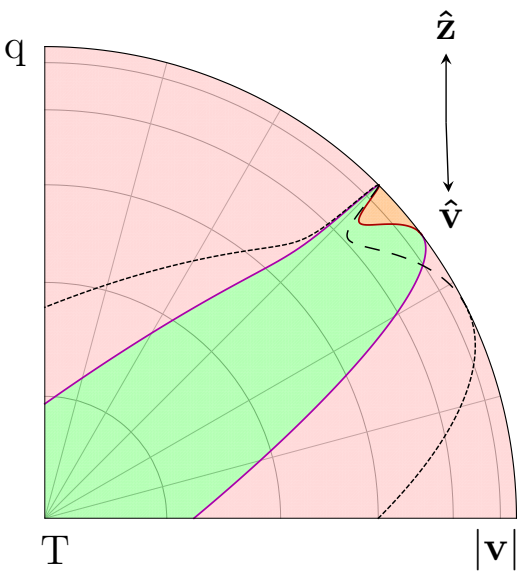

(e) $\phi=-88^{\circ}$

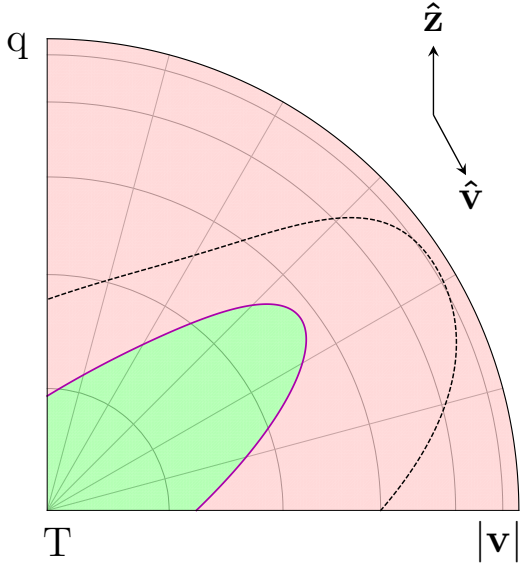

(c) $\phi=-60^{\circ}$

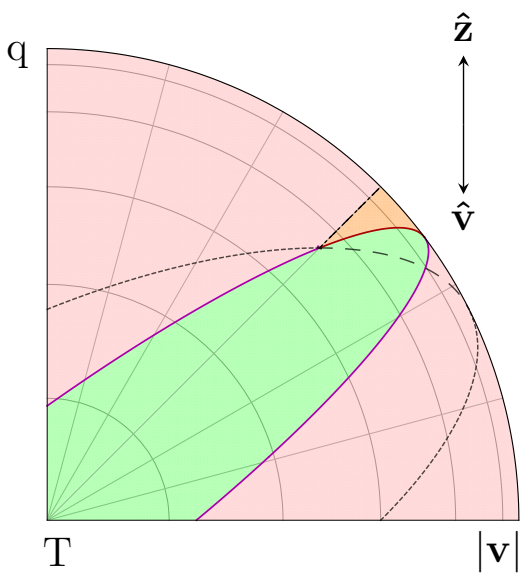

(f) $\phi=-90^{\circ}$

Figure 4.4: Orthographic projections of the positive octant of the sphere onto the $|\mathbf{v}|-q$ plane for different values of angle of translation at Stokes flow $\left(R_{\nu}=0.5\right)$.

We see that as $\phi$ decreases from $90^{\circ}$ the one end blowup region increases in size. At one point the one end blow up region touches the outer boundary and then two end blow up region appears. Only when the velocity of translation is almost collinear with the body force, the two end blow up region is seen in the parameter space. We also note that the two points where the boundary of one end blow up intersects the $q-T$ and $T-|\mathbf{v}|$ axes are not changing with the angle of translation. Also note that for $\phi=-90^{\circ}$ the line $|\mathbf{v}|=q$ is a pathological case in our parameter space because the rescaling for our system breaks down. This can be seen in figure (f) of the above charts.

For the case of vertical translation it turns out that the region for one end blowup is an 
ellipse on the projected $|\mathbf{v}|-q$ plane. This can be shown in the following way; the boundary of the region is given by the contour:

$$
\begin{aligned}
& k-\bar{T}=0 \\
& \Longrightarrow 1+R_{\nu} \bar{U}^{2}+\left(R_{\nu} \bar{W}+\bar{Q}\right)(\bar{W}+\bar{Q})-\bar{T}=0 \\
& \Longrightarrow 1+R_{\nu} \bar{W}+\bar{Q}=\bar{T} \text { Since } \phi=90^{\circ}, \bar{U}=0 \text { and } \bar{W}+\bar{Q}=1 \\
& \Longrightarrow 1+\frac{R_{\nu}|\mathbf{v}|+q}{\Delta}=R_{\nu} \frac{\sqrt{1-q^{2}-|\mathbf{v}|^{2}}}{\Delta}
\end{aligned}
$$

After some manipulation of the above expression we get that the contour of the region is given by:

$$
|\mathbf{v}|^{2}\left[\left(1+R_{\nu}\right)^{2}+R_{\nu}^{2}\right]+4|\mathbf{v}| q\left(1+R_{\nu}\right)+q^{2}\left(4+R_{\nu}^{2}\right)=R_{\nu}^{2}
$$

This is an equation for an ellipse. The length of the major and the minor axis of the ellipse is determined by the anisotropy in the drag forces. We should also note that this ellipse on the orthographic projection corresponds to two small circles on the sphere.

\subsection{Effect of changing $R_{\nu}$ on the parameter space}

We have been talking about Stokes flow so far. But what is the effect of changing $R_{\nu}$ on the nature of the possible phase portraits? The range of $R_{\nu}$ that we are interested in is $[0,1]$. If we increase the value of $R_{\nu}$ we are decreasing the anisotropy in the drag forces. It turns out that at a given angle of translation, if we increase $R_{\nu}$, then the two end blow up region in the parameter space shrinks in size, and when the drag forces are perfectly isotropic we lose these solutions completely. This can be seen in the figure 4.5 . 


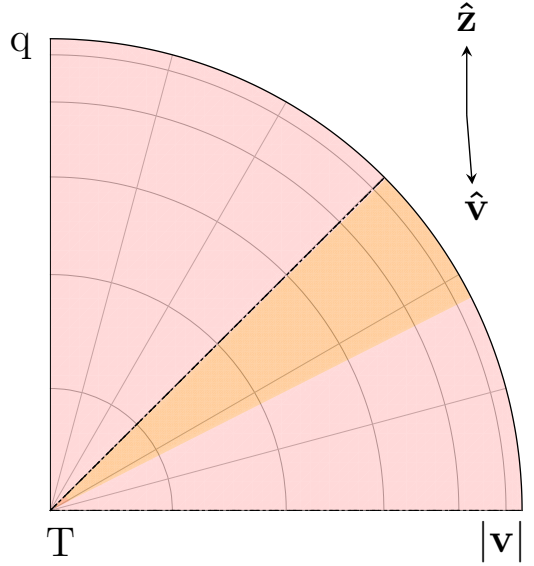

(a) $R_{\nu}=0$

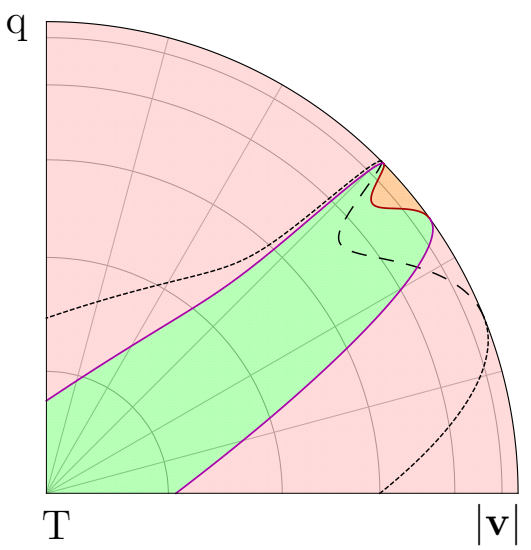

(d) $R_{\nu}=0.4$

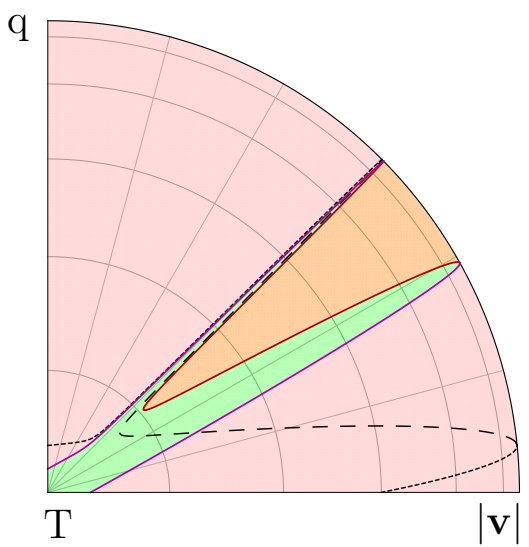

(b) $R_{\nu}=0.1$

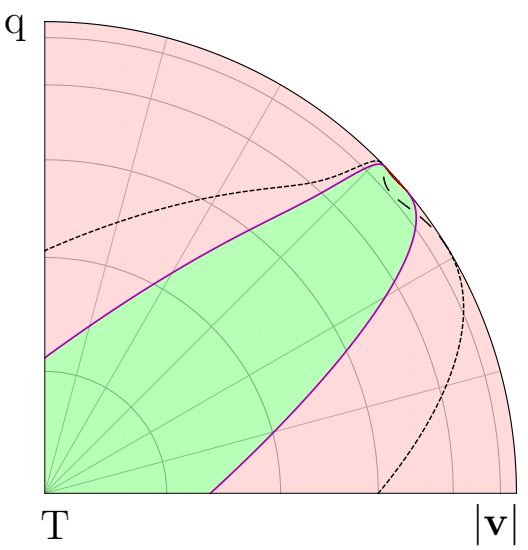

(e) $R_{\nu}=0.6$

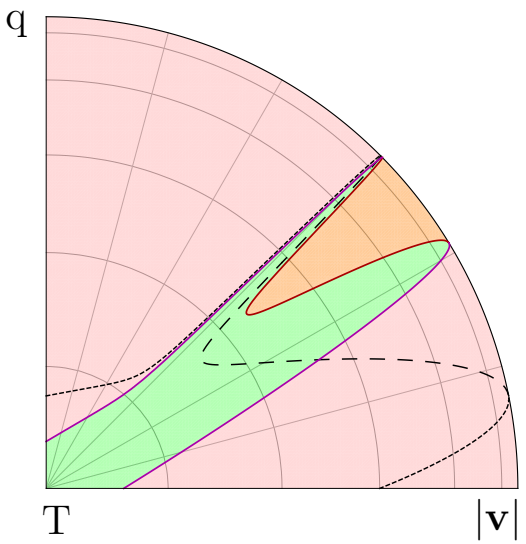

(c) $R_{\nu}=0.2$

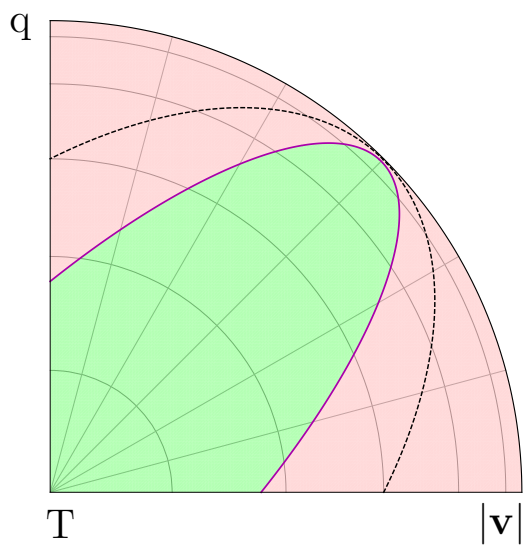

(f) $R_{\nu}=1$

Figure 4.5: Different regions on the $|\mathbf{v}|-q$ plane for different values of drag ratio at a constant angle of translation, $\phi=-85^{\circ}$

The above figures are for the case of a string translating at an angle of $-85^{\circ}$. This angle of translation was chosen as one can observe all the possible behaviors of phase portraits on the $|\mathbf{v}|-q$ plane. We observe that the two end blow up region disappears for isotropic drag forces. On the other limit $\left(R_{\nu}=0\right)$ we do not have the one end blow up regime.

In the limiting case, $R_{\nu}=1$, the contour of the one end blowup region is given by: 


$$
\begin{array}{rlr}
k-\bar{T} & =0 & \\
\Longrightarrow 1+R_{\nu} \bar{U}^{2}+\left(R_{\nu} \bar{W}+\bar{Q}\right)(\bar{W}+\bar{Q})-\bar{T} & =0 & \\
\Longrightarrow 1+\bar{U}^{2}+(\bar{W}+\bar{Q})^{2}-\bar{T} & =0 \quad \text { since } R_{\nu}=1 \\
\Longrightarrow \bar{T} & =2 & \\
\Longrightarrow \frac{1-q^{2}-|\mathbf{v}|^{2}}{\Delta^{2}} & =4 &
\end{array}
$$

On substituting $\Delta$ and doing some algebraic simplification we get that the equation for the contour is:

$$
5|\mathbf{v}|^{2}+8|\mathbf{v}| q \sin \phi+5 q^{2}=1
$$

Here $\phi$ is the angle of translation with respect to the positive $\hat{\mathbf{x}}$ axis as mentioned earlier. The above equation is that of an ellipse. So the green region for isotropic drag forces is elliptical in the projected $|\mathbf{v}|-q$ plane and it corresponds to two small circles on the sphere. 


\section{Chapter 5}

\section{Heteroclinic Connections}

We have so far talked about the visualization of the parameter space and different kinds of phase portraits. In this chapter we specifically talk about heteroclinic connections. We put forward a discussion on the geometry and stress distribution of the curves obtained from heteroclinic connections.

\section{Curve Geometries}

A heteroclinic connection is a phase trajectory connecting the fixed points of the dynamical system. These phase trajectories reach the fixed points asymptotically. We have already discussed that the curves obtained from these connections are of infinite length. However we use a method of cut off, as outlined in section 3.2, and represent a curve that is of length one.

We shall only consider Stokes flow (where $R_{\nu}=0.5$ ) in this chapter. We shall look at how these curves behave at different angles of translation with different velocities. The shades of black in the curves indicate the magnitude of the velocity. The darker the shade the higher is the velocity of translation.

Figure 5.1 shows configuration of a string translating in the upward direction. The curves have a shape that resembles a catenary. The orbits of the phase portraits are symmetric. Because of this, the curves obtained on integrating these orbits are also symmetric. The length of all the curves shown in the figure are unity. However the curves can actually extend to infinity and that has been indicated by the dashed lines at the end of the curves. The stress distribution along the curves shows that the curves are in tension. For an infinitely long curve, the tension at the end of the curves is also infinite in the absence of axial flow. 

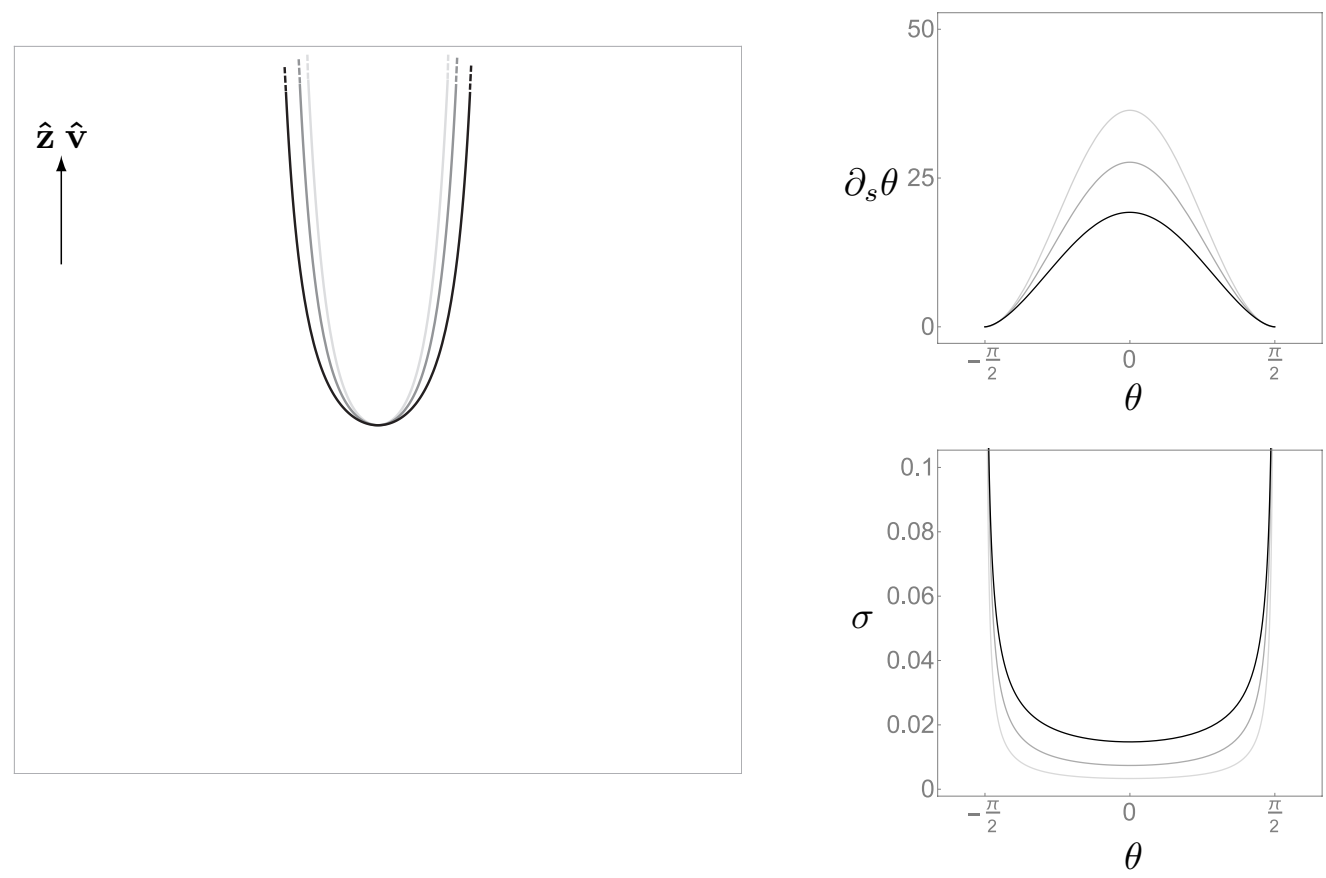

Figure 5.1: Curves (left) and the phase trajectories with the stress distribution (right) for upward translation for Stokes flow $\left(R_{\nu}=0.5\right)$. Here $|\mathbf{v}|=0.1,0.5,0.95 ; q=0.99,0.87,0.31$; $T=0$ and $m=1$.

Figure 5.1 shows three orbits from which the curves where obtained. Each of these orbits are accompanied by a set of another phase trajectories (not shown in the figure) that are just the reflection of these about the horizontal plane. Curves obtained from the orbits that are in the lower half of the plane will be rotations of the curves shown in figure 5.1 about an axis normal to the plane. Those curves will be in compression. Since we are considering perfectly flexible filaments curves under compression are not stable solutions of the problem.

The following figures 5.2-5.5 are for translation of the string at different angles. The direction of translation is shown in the figure and is also explicitly provided in the caption. We see an interesting behavior. When the velocity of translation is small, the body force dominates. The body force tries to orient the curve like it would do for a hanging chain and we find solutions that have a shape like a catenary. However when the velocity increases in magnitude, the curves reorient themselves in the direction of translation. 

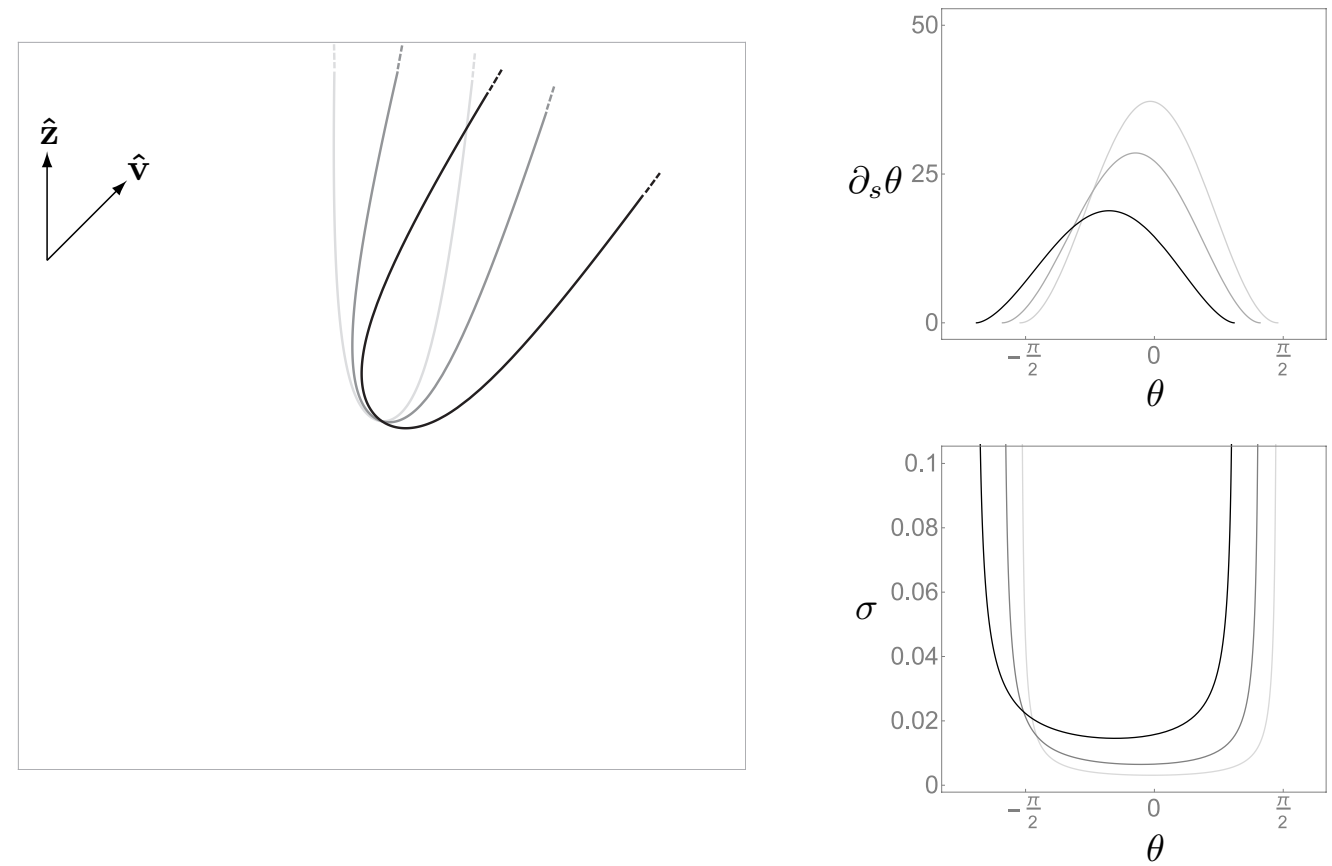

Figure 5.2: Curves (left) and the phase trajectories with the stress distribution (right) for Stokes flow $\left(R_{\nu}=0.5\right)$. Here $|\mathbf{v}|=0.1,0.5,0.95 ; q=0.99,0.87,0.31 ; T=0 ; m=1$. Angle of translation $\phi=45^{\circ}$.
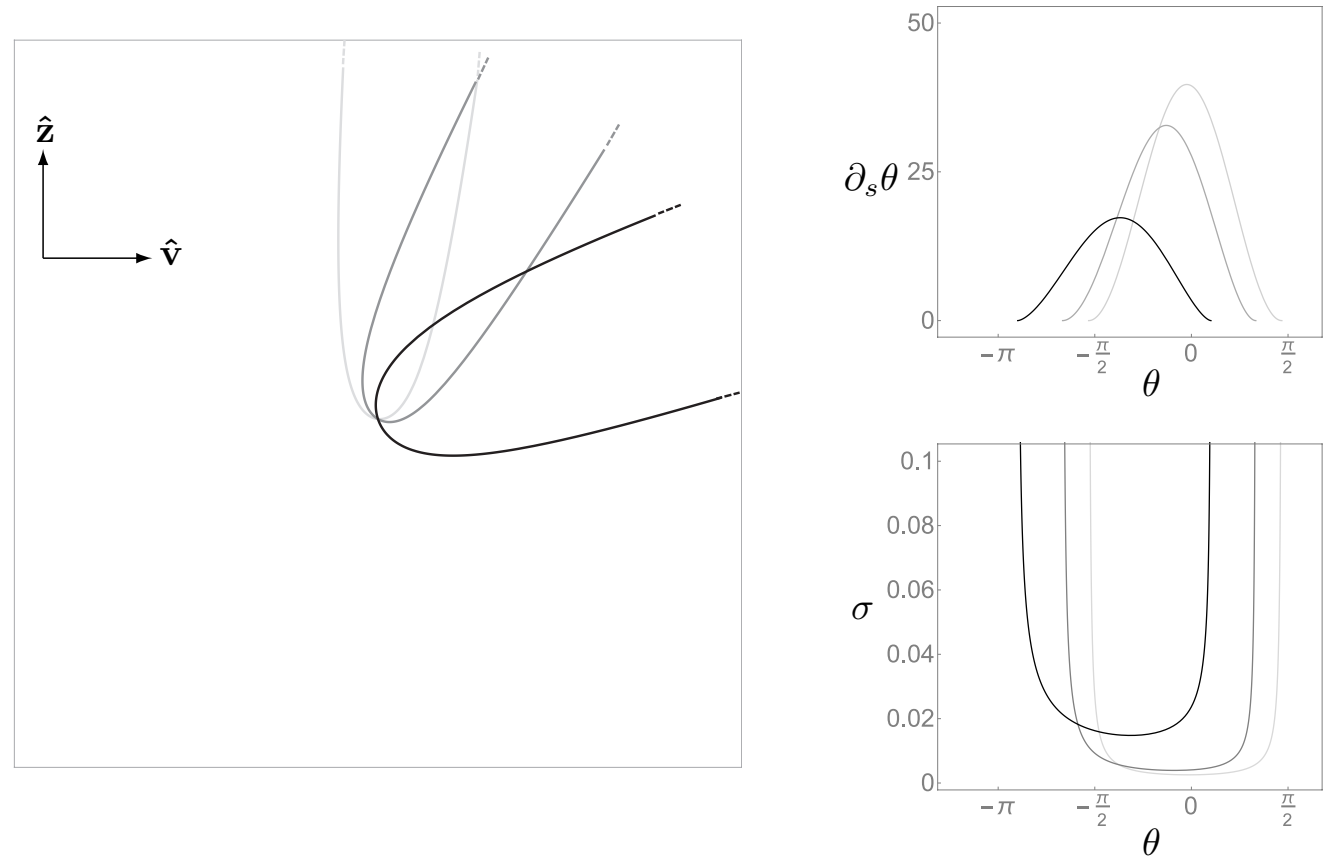

Figure 5.3: Curves (left) and the phase trajectories with the stress distribution (right) for Stokes flow $\left(R_{\nu}=0.5\right)$. Here $|\mathbf{v}|=0.1,0.5,0.95 ; q=0.99,0.87,0.31 ; T=0 ; m=1$. The curves are translating in the horizontal direction $\left(\phi=0^{\circ}\right)$. 

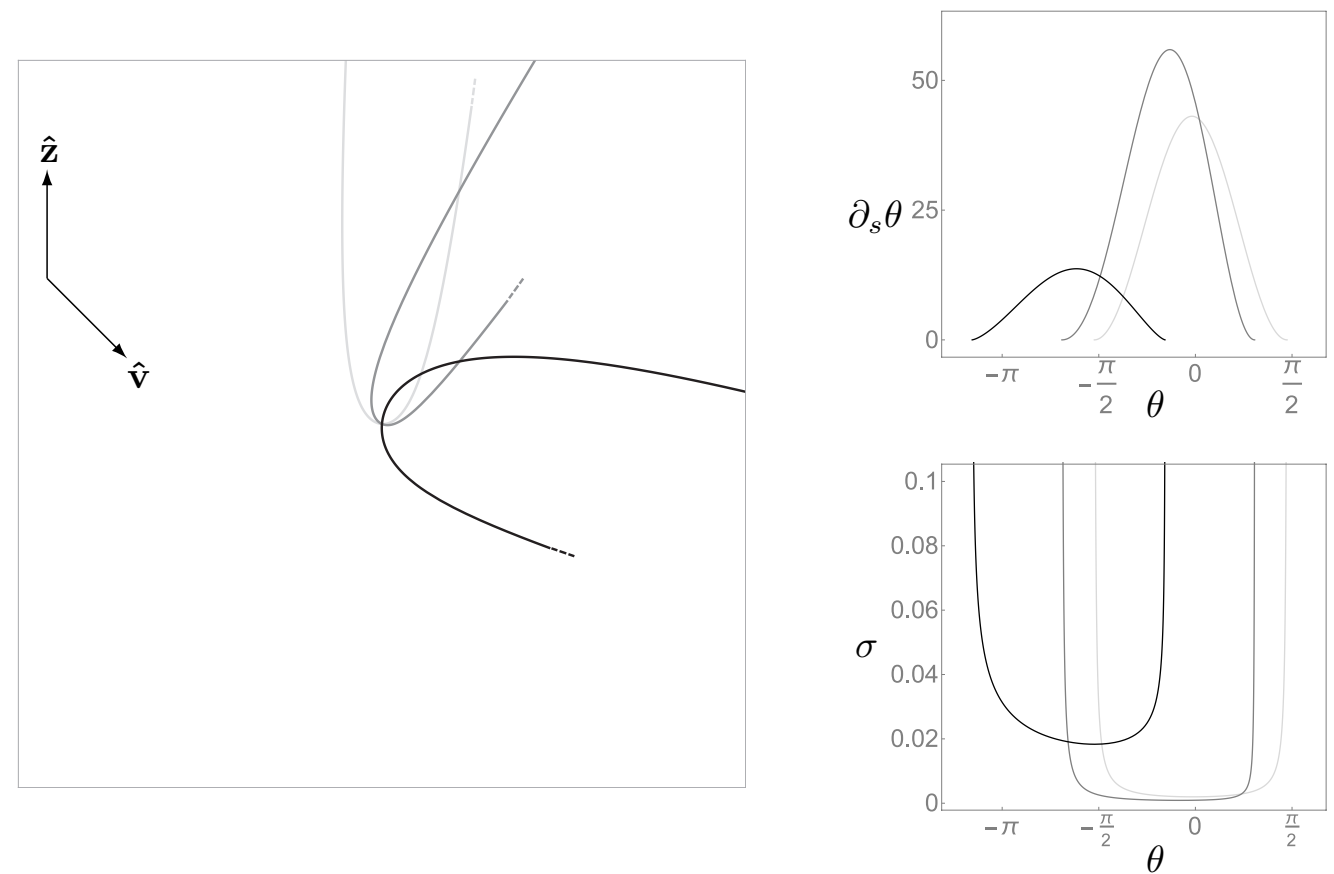

Figure 5.4: Curves (left) and the phase trajectories with the stress distribution (right) for Stokes flow $\left(R_{\nu}=0.5\right)$. Here $|\mathbf{v}|=0.1,0.5,0.95 ; q=0.99,0.87,0.31 ; T=0 ; m=1$. Angle of translation $\phi=-45^{\circ}$.
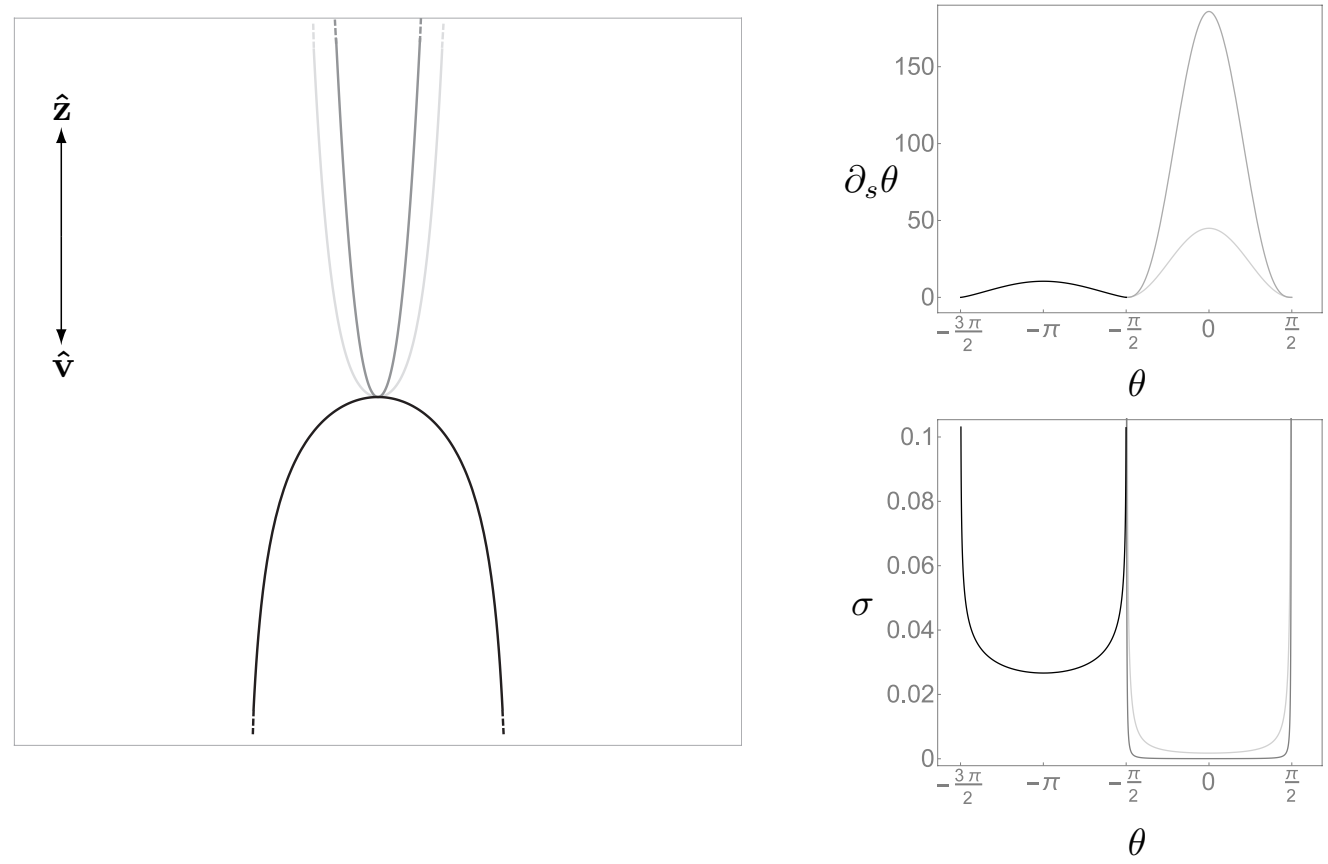

Figure 5.5: Curves (left) and the phase trajectories with the stress distribution (right) for Stokes flow $\left(R_{\nu}=0.5\right)$. Here $|\mathbf{v}|=0.1,0.5,0.95 ; q=0.99,0.87,0.31 ; T=0 ; m=1$. The velocity of translation is almost collinear with the body force with, $\phi=-90^{\circ}$. 
The asymmetry that we see in the curves is a result of the asymmetry in the phase portrait. Figure 5.5 corresponds to the almost downward translation of the string. In this figure we see that when the velocity is sufficiently high the curve flips and gets inverted.

Effect of axial flow: The addition of axial flow to the problem causes further asymmetry in the phase portraits and thus also in the curves. In section 3.2 we have already discussed that the phase trajectories are not invariant under the change of sign of the axial flow. The curves for two different directions of tangential flow look different.

As mentioned in the discussion on parameter space in section 4.1, the right way to appreciate the effect of axial flow is to move along one of the radial lines in the projected view. For this we fix the ratio $\frac{|\mathbf{v}|}{q}$ and then vary $T$. For the present case, we fix the ratio between the velocity and the body force at 0.3 and then look at two equal but opposite values of the tangential velocity.

The figure below illustrates this case. In figure 5.6 the black curve is one without any axial flow. As we add axial flow in either direction, we see that the phase trajectories become asymmetric. In general, curves with tangential velocity tend to look like 'pointed fingers'.
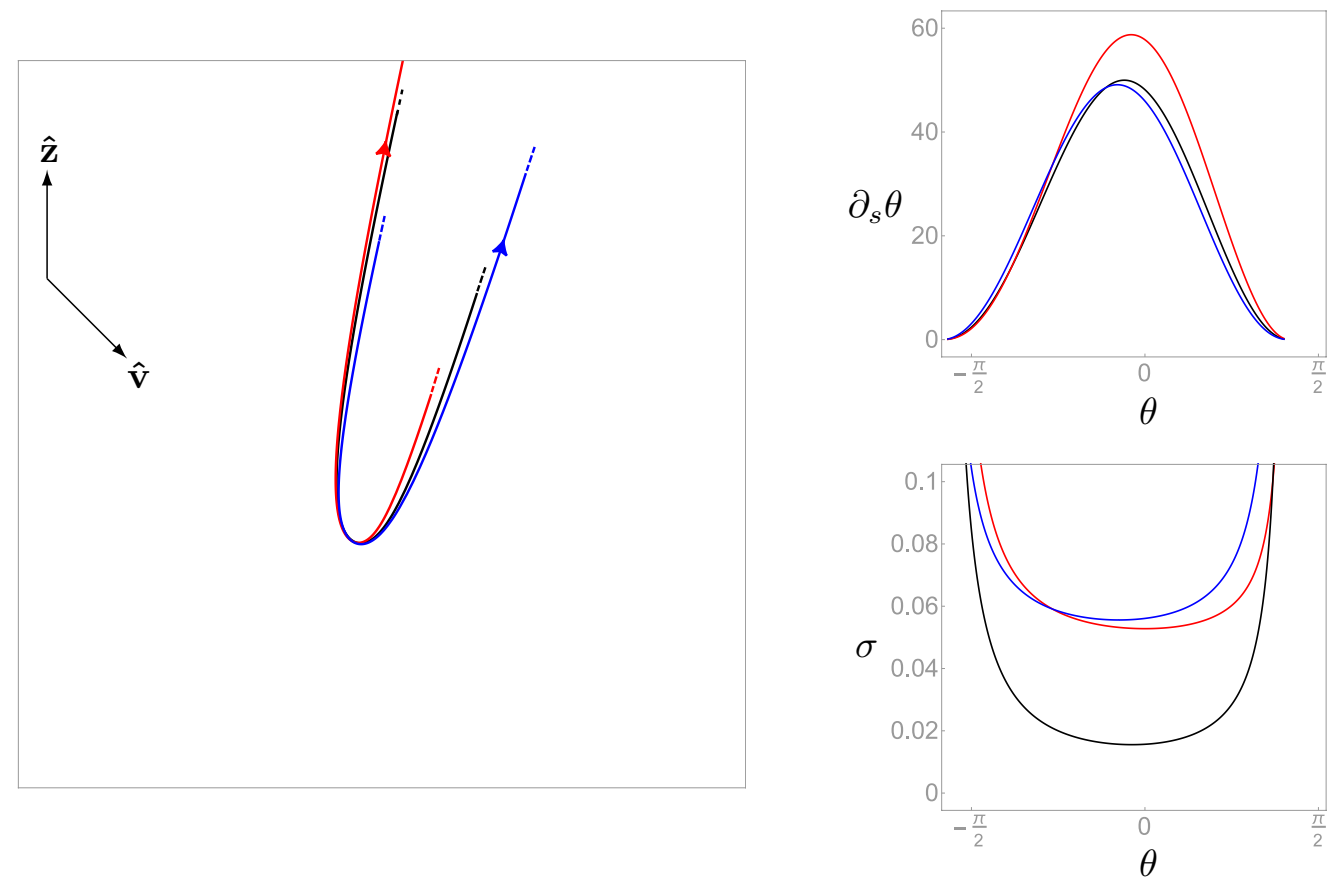

Figure 5.6: Curves (left) along with the phase portrait and thr stress distribution (right) for Stokes flow $\left(R_{\nu}=0.5\right)$. Here $|\mathbf{v}|=0.28,0.29,0.28 ; q=0.94,0.96,0.94 ; T=-0.2,0,0.2$; $m=1$. Angle of translation $\phi=-45^{\circ}$. For the cut through the parameter space we had, $\frac{|\mathbf{v}|}{q}=0.3$. 


\section{Chapter 6}

\section{Sedimentation of flexible filaments}

We have already discussed the problem of sedimentation at low Reynolds number in our introduction. We have also pointed out that most of the work considering the sedimentation of filaments has been numerical and has been centered around filaments with bending resistance ([41], [45], [47]). For a sedimenting filament the appropriate boundary conditions are stress free ends and axial flow $T=0$. So any solution to our problem for a finite length curve where the stress vanishes at both ends of the filament will correspond to sedimenting solutions. In this chapter we discuss possible sedimenting solutions for purely flexible filaments.

Let us consider an example for Stokes flow $\left(R_{\nu}=0.5\right)$. When the velocity of translation is collinear with the body force (downward translation) then there is a region in the parameter space, indicated by the orange color in figure 4.2 that corresponds to the two end blow up phase trajectories. As mentioned earlier in section 3.2, the curves obtained from these phase trajectories are of finite length and we can rescale them to be of length one. Let us look at the geometry of the curves and the stress distribution for the blow up phase trajectories.

Figure 6.1 shows the geometry of the curves obtained for this case. The stress distribution shows that the curves are in tension and the stress vanishes at the end. In fact, the stress at the ends is given by: $\sigma_{e n d s}=m T^{2}$. Since the axial flow $T$ is zero, the stress vanishes at the end. 

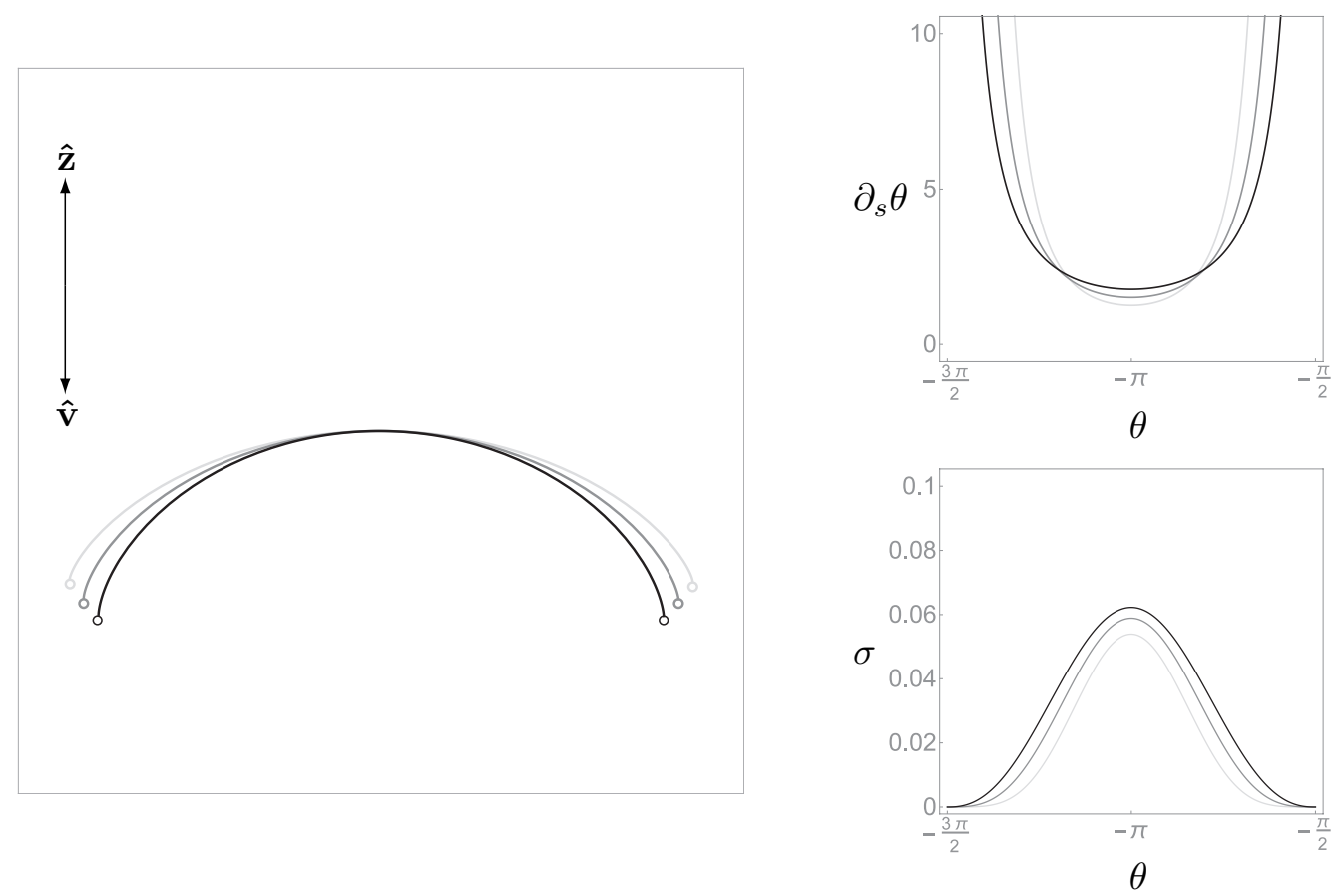

Figure 6.1: Sedimentation solution for purely flexible filaments (left) and stress distribution (bottom right), corresponding to the two end blow up phase trajectories (top right) at Stokes flow $\left(R_{\nu}=0.5\right)$. We have, $|\mathbf{v}|=0.74,0.75,0.76 ; q=0.67,0.66,0.64$. Axial flow, $T=0$, $m=1$ and $\phi=-90^{\circ}$. The small circles at the end of the curves indicate that these curves are all of finite length in contrast to the infinite length curves obtained from the heteroclinic connections that has been shown by dashed line at the ends.

Since we have curves that are of finite length and are in tension with zero stress at the ends, these correspond to the sedimenting solutions for purely flexible filaments. One question that may be asked is what is the terminal velocity with which a filament of a given length will sediment? Our results suggest that there may be infinitely many solutions instead of one particular solution to the problem. This is because all the velocities chosen from the two end blow up region will give us a solution for sedimentation.

Terminal velocity for a sedimenting filament is determined by the balance of the net integrated drag force along the filament and the body force ([40],[41]). Since we have balanced forces locally, that in all our solutions the global drag force is also balanced by the body force, and there are infinitely many velocities of sedimentation for which this is true.

The other thing that is worth pointing out is that if $R_{\nu}=1$ then we do not have any region in our parameter space that supports two end blow up solutions. This suggests that the existence of the sedimenting solution in our system is tied to the fact that there is anisotropy in the drag forces. If the drag forces are isotropic, then we lose all these solutions. 
Length of the curves: In the case of perfectly downward translation, $\bar{U}=0$. So it follows:

$$
(\bar{W}+\bar{Q})^{2}=1
$$

If there is no axial flow $T$ in the system, then it turns out that we can explicitly write an expression for the length of curves that one will obtain by integrating a two end blow up phase trajectory. For that, first we should note that our dynamical system reduces down to:

$$
\partial_{s} \theta=C|\cos \theta|^{k}
$$

where the symbols have their usual meaning. The two fixed points of the lobe that we have been considering so far are: $-\frac{\pi}{2}$ and $\frac{\pi}{2}$. So the length of a curve obtained integrating along this orbit is given as:

$$
\begin{aligned}
& s=\int_{-\frac{\pi}{2}}^{\frac{\pi}{2}} \frac{d \theta}{C|\cos \theta|^{k}}, \\
& s=\frac{\sqrt{\pi} \Gamma\left(\frac{1-k}{2}\right)}{2 C \Gamma\left(1-\frac{k}{2}\right)}, \quad \text { Provided } k<1
\end{aligned}
$$

Here $\Gamma$ is the gamma function. The above integral is convergent only for $k<1$. For downward translation of the string, $k<0$ so the integral has a finite value indicating that the length of all these curves are finite.

Effect of axial flow: The effect of axial flow on the geometry of the curve is to create asymmetry, as seen in figure 6.2. We see that due to the asymmetry, the two end points of the string are not on the same horizontal level. 

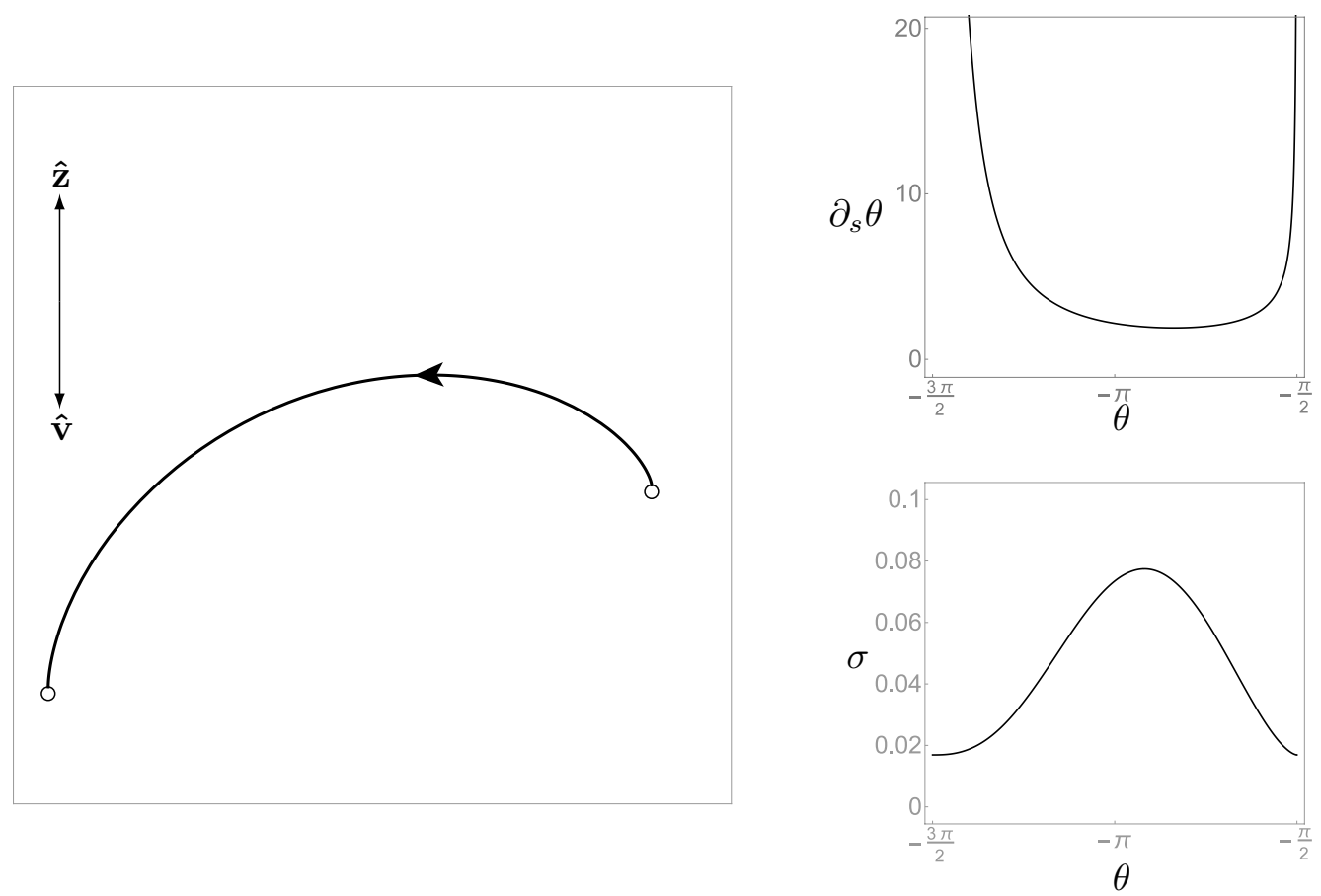

Figure 6.2: Curve (left) and the orbit with the stress distribution (left) for two end blow up phase portraits in Stokes flow $\left(R_{\nu}=0.5\right)$. Here $|\mathbf{v}|=0.76, q=0.64, T=0.13$ and $m=1$. The angle of translation $\phi=-90^{\circ}$.

The important thing to note from the figure is the stress distribution for this curve. Due to nonzero axial flow the stress at the ends of the curve is not zero, instead it is equal to $m T^{2}$. Since the stress is nonzero at the ends, this cannot be a solution for sedimenting filament. 


\section{Chapter 7}

\section{One end blow up and geometric special cases}

\subsection{One end blow up}

The third case that we would like to discuss is the case of one end blow up phase trajectories. Now we are in the green region of the parameter space. For a given orbit in the phase space there is one fixed point and one pole. As discussed in section 3.2 the curves obtained from these orbits are semi-infinite. The curve extends to infinity at the end of the fixed point and terminates at the end of the pole.

Just like before we indicate the finite end of the curve with a small circle and the dashed line indicate the end extending to infinity. The curve geometry along with the stress distribution is shown in figure 7.1. We also observe that the stress at the finite end is $m T^{2}$, and on the other end it is infinite. We will discuss in further detail how the stress at the end of a curve behaves in chapter 8 . 

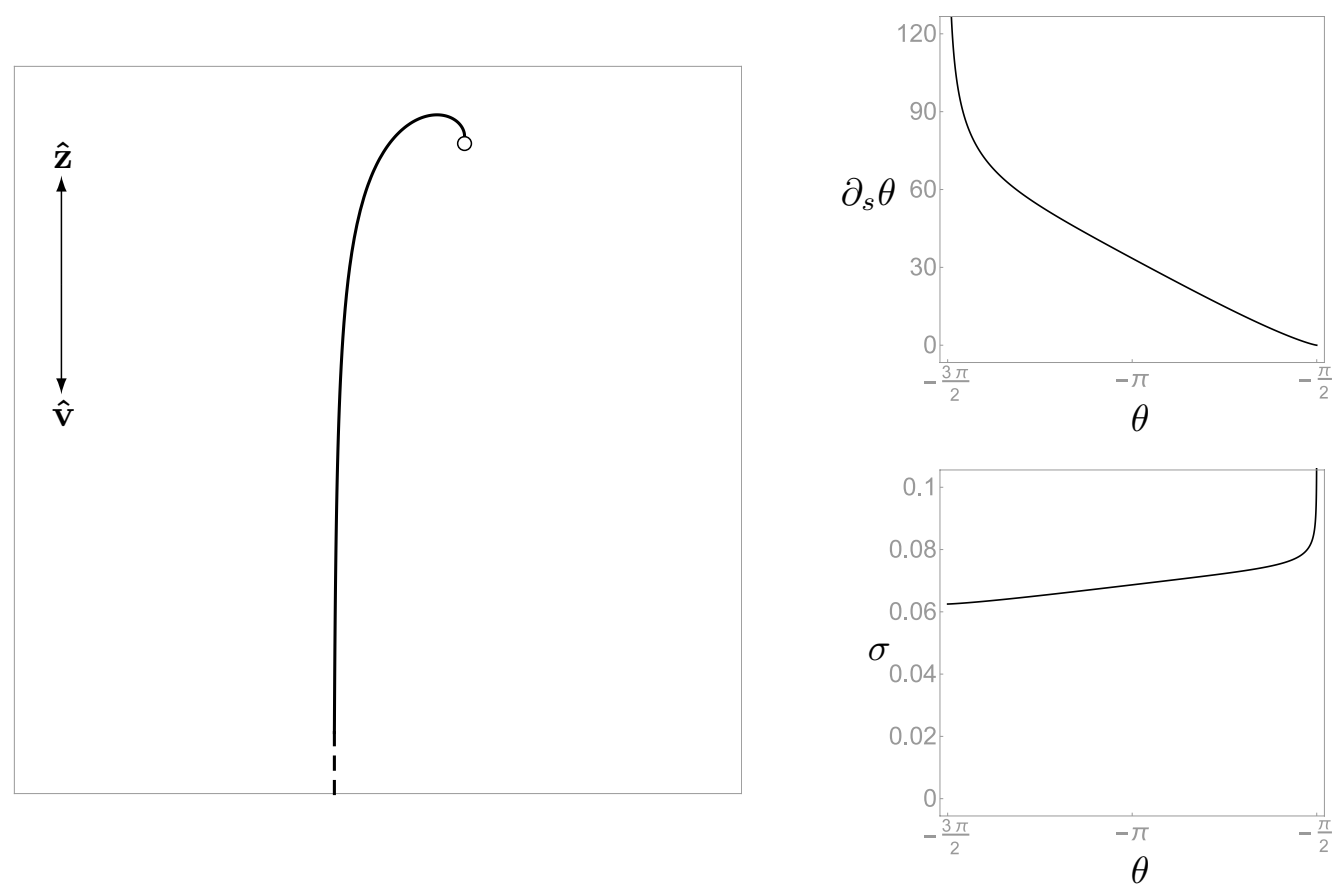

Figure 7.1: Curve (left) and the orbit with the stress distribution (left) for one end blow up phase portraits for $R_{\nu}=0.6$. We have, $|\mathbf{v}|=0.78, q=0.57, T=0.25$ and $m=1$. The angle of translation $\phi=-90^{\circ}$.

\subsection{Geometric Special Cases}

We have so far discussed the geometry of curves and the stress distribution arising from three different kinds of phase portraits. Now we focus on what happens at the boundary between the different phase portraits in our parameter space. We shall call these: geometric special cases.

\subsubsection{Phase portraits with doubled periodicity}

Here we discuss phase trajectories arising on the boundary of transition between heteroclinic to one end blow up and on the boundary between one end blow up and two end blow up. We show how these phase trajectories are $2 \pi$ periodic in these cases, and discuss the nature of the curves.

Looping Curves: Consider a cut along an iso-T line through the parameter space. While crossing from heteroclinic connections to one end blow up region (and vice versa) one en- 
counters the violet color boundary as shown in the previous orthographic projections. On this boundary two adjacent $\pi$ periodic heteroclinic orbits (or one end blow up phase trajectories) get connected to form a single $2 \pi$ periodic heteroclinic connection. The condition on the exponents for this behavior is $k-\bar{T}=0$.

For a given tangential velocity, angle of translation and $R_{\nu}$, we implement a Newton search to find the zero of the function $k-\bar{T}$ from which we obtain the magnitude of the velocity of translation. This determines the exact point on the violet boundary for which we have the $2 \pi$ periodic heteroclinic connection.
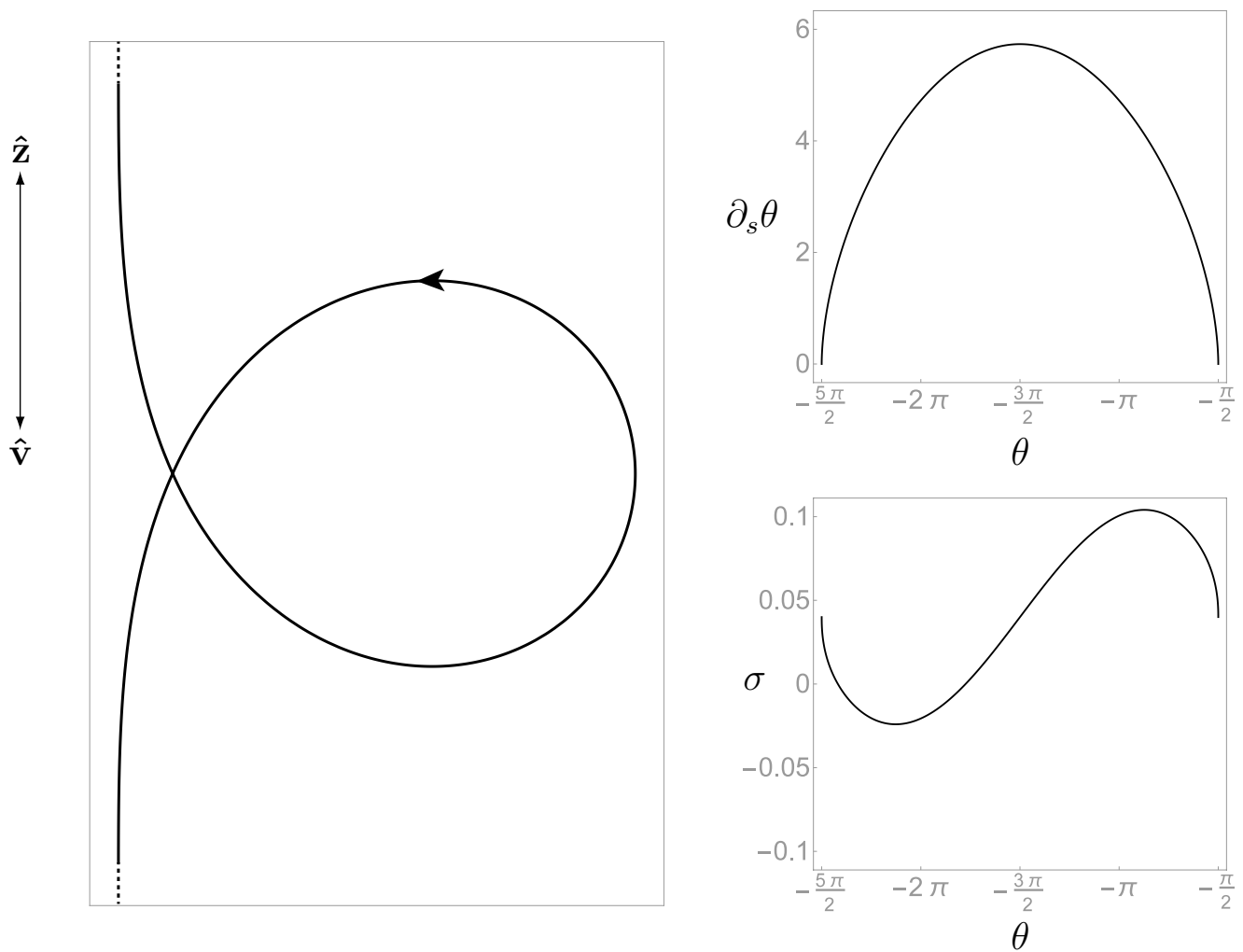

Figure 7.2: A looping curve (left) from $2 \pi$ periodic heteroclinic connection (top right) with the stress distribution (bottom right) for $R_{\nu}=0.4$. Here $T=0.2, \mathbf{v} \approx 0.82, q=0.53$ and $m=1$. The angle of translation, $\phi=-90^{\circ}$.

Figure 7.2 shows the geometry of a curve and its stress distribution as obtained from the $2 \pi$ periodic phase trajectory. We see that the curve forms a loop and its ends go to infinity at both ends, as expected for a heteroclinic connection. This is a self intersecting curve and we do not expect to find these in practical problems as equilibrium solutions. It can be seen from the figure that the curve is symmetric about the horizontal plane. One can think that this geometry is a combination (or addition) of two curves each obtained from two adjacent 
$\pi$ periodic heteroclinic (or one end blow up) trajectories. Since we used unit length curves for $\pi$ periodic lobes, the curve shown here has a length of two units.

We observe that the stress distribution is also symmetric about $m T^{2}$. A portion of the upper half of the curve is in compression and the lower half is in tension. A purely flexible curve cannot resist compressive forces. That is why we believe that these solutions are not stable.

$2 \pi$ periodic merged blowup: Now consider another iso- $T$ cut in parameter space where we cross from one end blow up region to the two end blow up region (or vice versa). In this transition one encounters the maroon colored boundary as shown in previous orthographic projections. On this boundary two adjacent $\pi$ periodic one end blow up (or two end blow up) phase trajectories get merged to form a single $2 \pi$ periodic two end blow up phase trajectory. The condition on the exponents for this behavior is $k+\bar{T}=0$. These boundary points are determined by a similar Newton search method described previously where we now look for zero of the function $k+\bar{T}$.
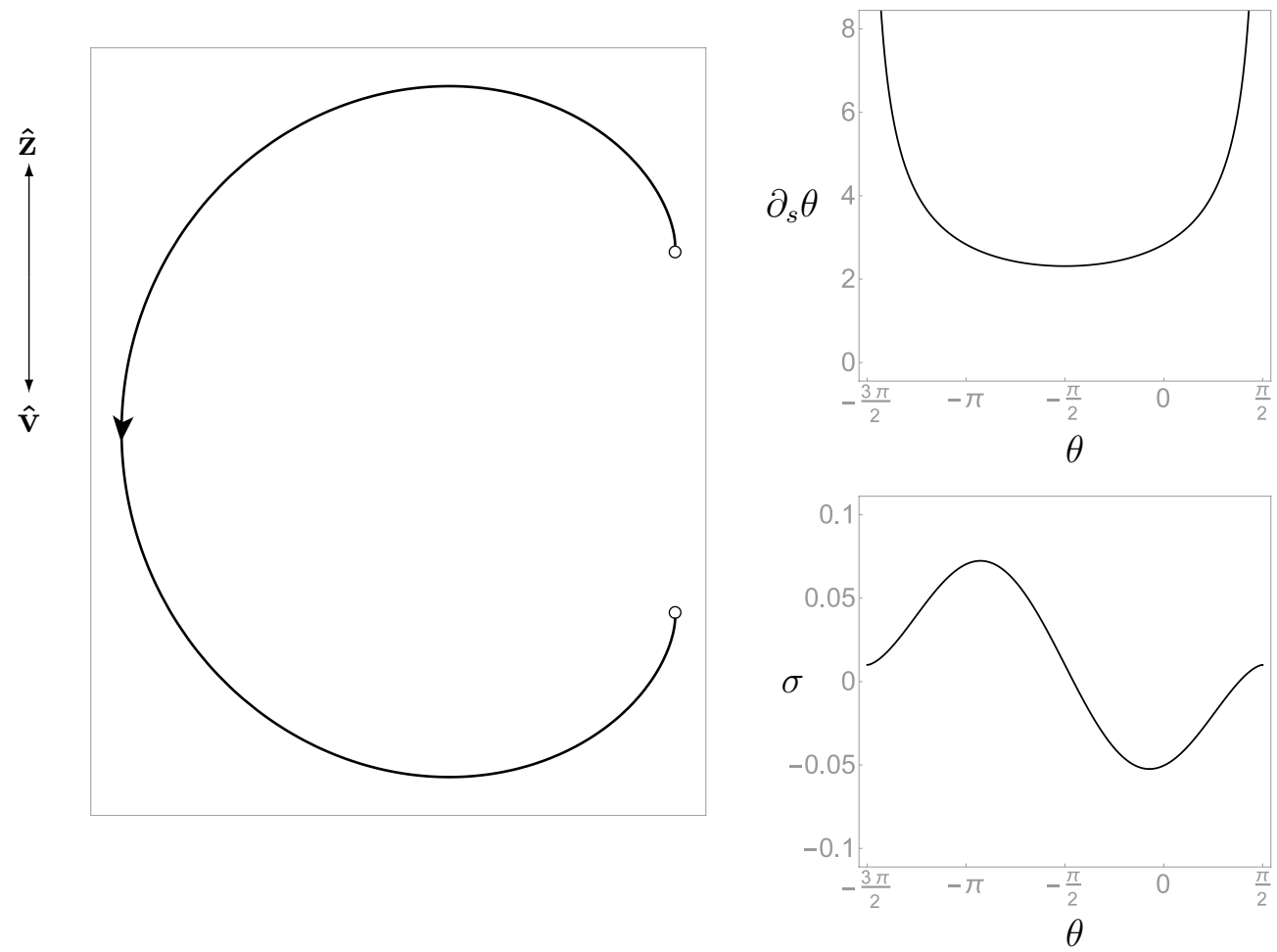

Figure 7.3: Finite length curve (left) obtained from $2 \pi$ periodic blowup phase portrait (top right) with the stress distribution (bottom right) for Stokes flow $\left(R_{\nu}=0.5\right)$. Here $T=0.1$, $|\mathbf{v}| \approx 0.78, q=0.61$ and $m=1$. The angle of translation $\phi=-90^{\circ}$.

Just like the previous case of looping curves this curve is symmetric about the horizontal 
plane. It can also be thought as a combination (or addition) of two curves obtained from adjacent $\pi$ periodic one end blow up (or two end blow up) trajectories. Like the looping curves the curve shown in figure 7.3 has a length of two units.

The stress distribution like before is symmetric in the figure about $m T^{2}$. In this case, a part of the lower half of the curve is in compression, as seen in the figure 7.3. It follows from the earlier discussion that due to compressive stress, these solutions are unstable.

\subsubsection{Circle and Logarithmic Spirals}

We have already discussed the special cases having $2 \pi$ periodic phase portraits. Both the cases described above appeared where tangential velocity was nonzero. Let us now consider the case where there is no axial flow.

Consider the case shown in figure 7.4. For the choice of parameters indicated in the figure, all possible regions in parameter space are present in the orthographic projection. Now when $T=0$, we are moving along the outer most arc of our projection. The two end blow up region appears in this case when we have $k<0$ and the heteroclinic connections correspond to $k>0$. The special case that we are going to talk about in this section is when we have $k=0$. This special case can be observed twice as we move along the $T=0$ iso line. As we increase the velocity of translation, the first special solution is observed for the transition to the two end blow up regime from heteroclinic connections. The second special case is observed for the transition to heteroclinic connections from two end blow up trajectories. These two cases have been indicated in figure 7.4 by two small circles on the $T=0$ boundary.

It is obvious from the discussion and the figure of the parameter space that this special case can exist only when the two end blow up region (colored orange) exists. For higher values of $R_{\nu}$, or a different angle of translation, we may not have the orange region in the parameter space. In that case this special solution will not be encountered. However for perfectly downward translation at $R_{\nu}=1$ the one end blow up region will just touch the boundary $(T=0 \mathrm{arc})$ and there will be one special solution of a circle instead of two special cases as seen in figure 7.4. 


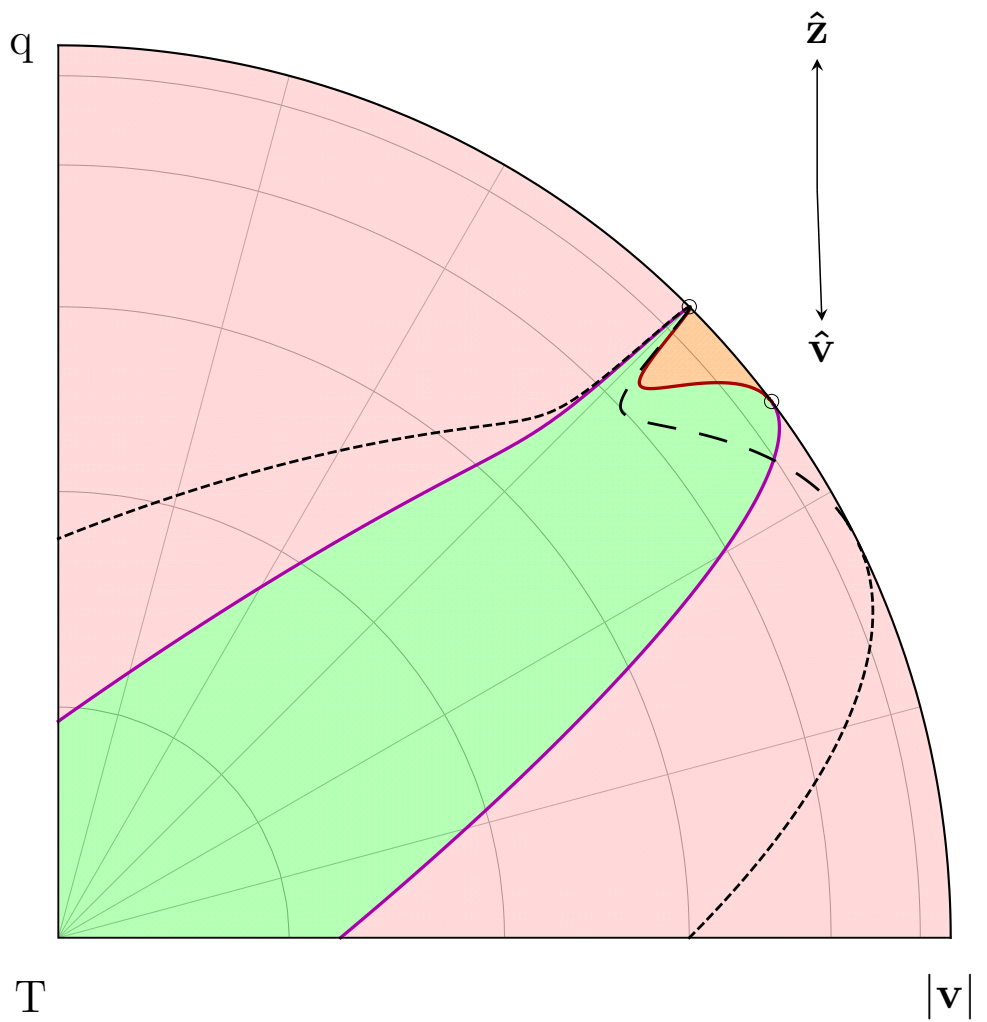

Figure 7.4: The two points corresponding to the special case of spirals for Stokes flow $\left(R_{\nu}=0.5\right)$. The angle of translation $\phi=-88.5^{\circ}$.

In the absence of axial flow, when we have $k=0$, our dynamical system reduces down to:

$$
\partial_{s} \theta=C e^{a \theta}
$$

where $a=\bar{Q} \bar{U}\left(1-R_{\nu}\right)$. Depending on the value of $a$ we can find two kinds of solutions. This is discussed below.

Logarithmic spiral: When the constant $a$ of the reduced dynamical system given by eqn (7.1) is nonzero, we have logarithmic spirals as solutions. We can integrate the dynamical system once more and can obtain a closed form solution for the geometry and stress distribution along the curve. 

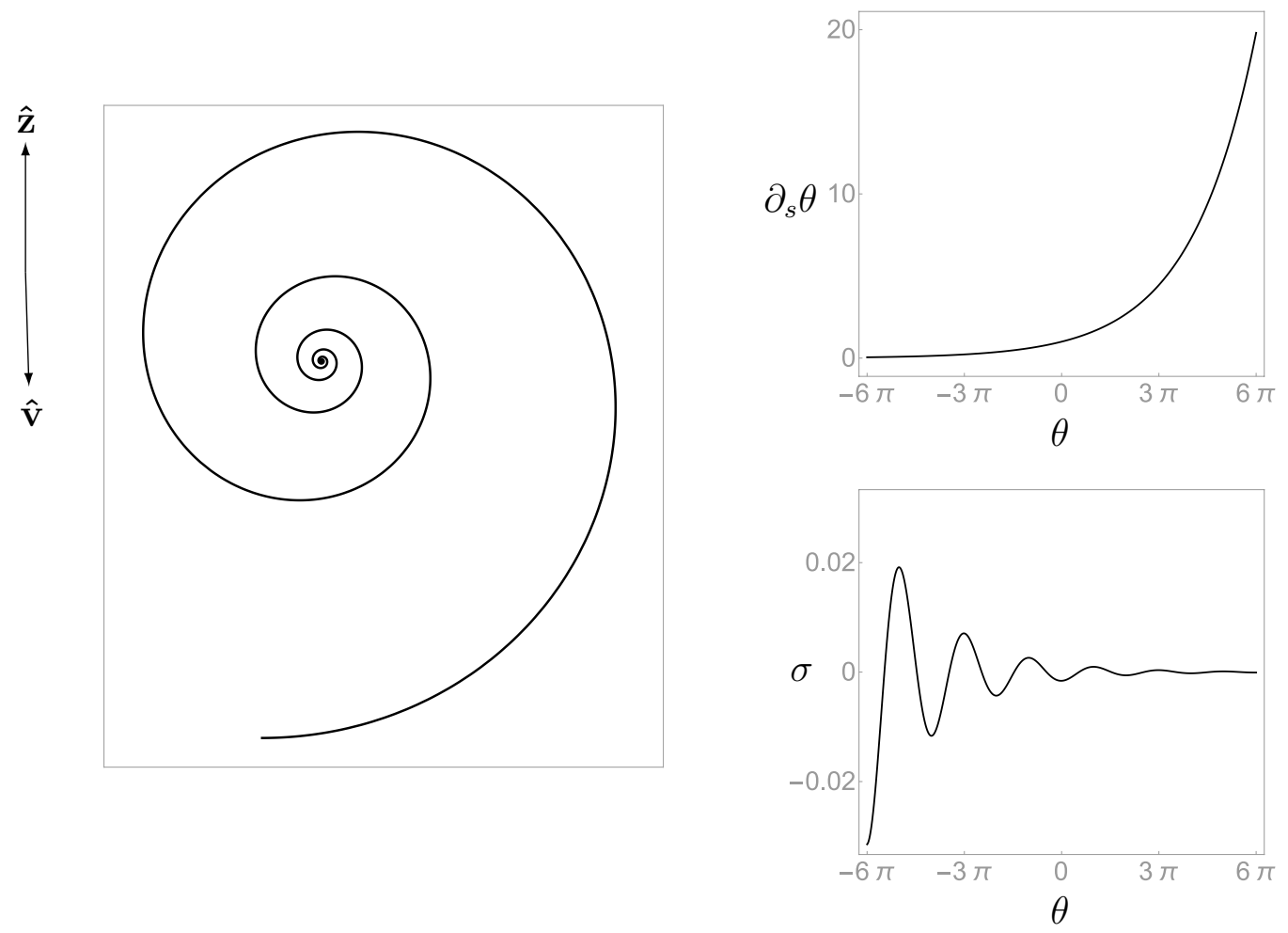

Figure 7.5: Logarithmic spiral as a special solution at Stokes flow $\left(R_{\nu}=0.5\right)$. Here we have: $T=0,|\mathbf{v}| \approx 0.79, q=0.61$ and $m=1$. The angle of translation $\phi=-88.5^{\circ}$.

The solution for this case can be written as:

$$
\begin{aligned}
x & =\frac{e^{-a \theta}}{C\left(a^{2}+1\right)}[\sin \theta-a \cos \theta]+x_{i n} \\
z & =-\frac{e^{-a \theta}}{C\left(a^{2}+1\right)}[a \sin \theta+\cos \theta]+z_{i n} \\
\sigma & =\frac{-\mathbf{v} \cdot \hat{\mathbf{x}} \sin \theta+(\mathbf{v} \cdot \hat{\mathbf{z}}+q) \cos \theta}{C e^{a \theta}}
\end{aligned}
$$

Here we observe the stress distribution to be an exponentially damped sine function. The stress oscillates between tension and compression. Since the curve is in compression, these are unstable solutions. 
Circle: When the curve is translating perfectly downwards, we have $\bar{U}=0$. The dynamical system represented in eqn (7.1) reduces down to:

$$
\partial_{s} \theta=C
$$

The solution to this is a circle with radius $\frac{1}{C}$. This is shown in figure 7.6.
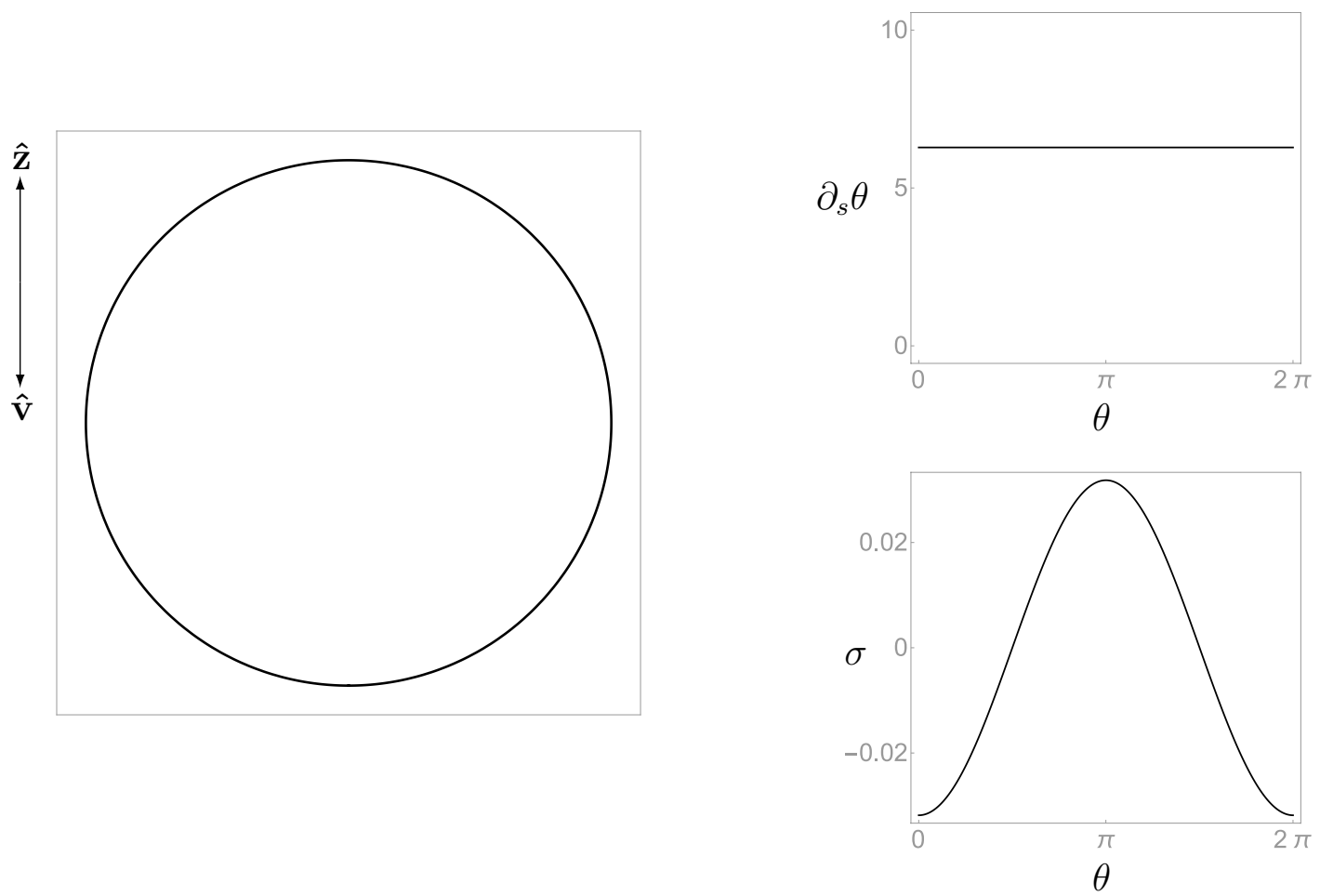

Figure 7.6: Circle as a special solution at Stokes flow $\left(R_{\nu}=0.5\right)$. Here we have: $T=0$, $|\mathbf{v}|=0.8, q=0.6$ and $m=1$. The angle of translation $\phi=-90^{\circ}$.

The stress distribution in the circle is a sine function where the lower half of the circle is in compression. Circle is the only periodic curve arising from the first order dynamical system. This is also an unstable sedimentation solution. 


\section{Chapter 8}

\section{Strange Behavior of the stress}

We have so far discussed different kinds of phase portraits, special cases and different geometries of curves. We have also shown the stress distribution with the tangential angle for these curves. However, in the presence of axial flow the stress behavior is nontrivial.

Our intuition suggests that if we have a curve that is infinitely long, the stress at the end of the curve goes to infinity. On the other hand if the curve is finite we should have finite stress at both ends. However this intuition is not always reliable. It turns out that it is possible to have curves with infinite lengths but finite stress at the ends. The conditions for this behavior are governed by the nature of the exponents of the different terms in our dynamical system. A detailed derivation of these conditions for different stress behaviors can be found in Appendix D. In this chapter we provide a qualitative description.

\subsection{Stress regions in the parameter space}

The octant of the sphere shown previously has been brought up here again. Along with the regions indicating the phase portraits, we also show two different dashed lines on the sphere. These lines separate different stress behaviors at the end of a curve. 


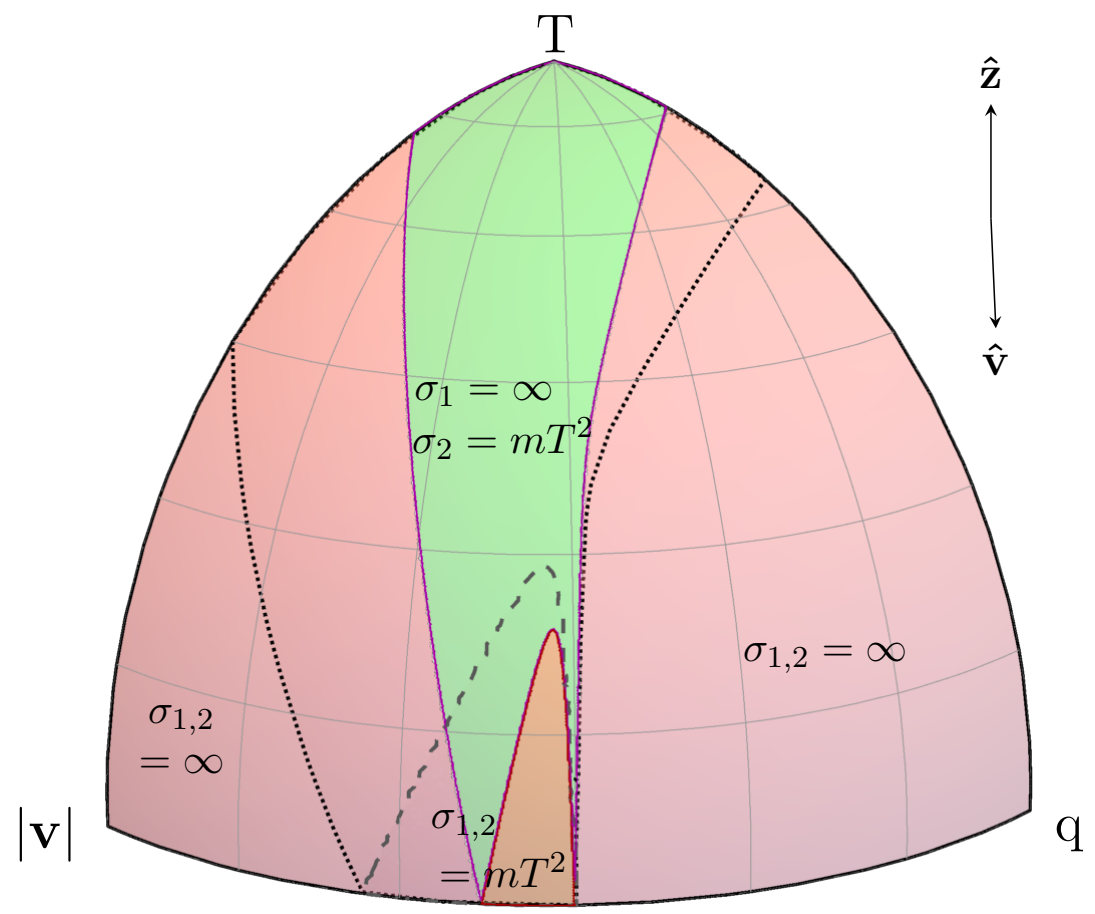

Figure 8.1: Different region of stress behavior in the octant of the sphere at Stokes flow $\left(R_{\nu}=0.5\right)$. The large dashed line corresponds to stress transition at the leading end $\left(l_{1}\right)$. The short dashed line corresponds to stress transition at the trailing end $\left(l_{2}\right)$. The angle of translation $\phi=-88^{\circ}$.

The stress at the end of a curve is governed by two expressions:

$$
\begin{aligned}
& l_{1}=1-k-\bar{T} \\
& l_{2}=1-k+\bar{T}
\end{aligned}
$$

The sign of these expressions determine the stress at the end of the curve. We list down the possible scenarios in form of a table:

All the solutions corresponding to the heteroclinic connections (pink region on the sphere) yield curves of infinite lengths. The curves obtained from the one end blow up trajectories are semi-infinite. From figure 8.1 we see that the stress at the end of a string is either $m T^{2}$ or it blows up to infinity. However on the boundary of this stress transition (dashed lines) one encounters a finite value of stress at one end that is different from $m T^{2}$. We observe from 8.1 that there are regions in the parameter space where one can have infinitely long 


\begin{tabular}{|c|c|c|}
\hline Conditions & Stress at $\theta_{1}^{*}\left(\sigma_{1}\right)$ & Stress at $\theta_{2}^{*}\left(\sigma_{2}\right)$ \\
\hline$l_{1}>0$ and $l_{2}>0$ & $m T^{2}$ & $m T^{2}$ \\
\hline$l_{1}<0$ but $l_{2}>0$ & $\infty$ & $m T^{2}$ \\
\hline$l_{1}<0$ and $l_{2}<0$ & $\infty$ & $\infty$ \\
\hline$l_{1}=0$ or $l_{2}=0$ & Special case & Special case \\
\hline
\end{tabular}

Table 8.1: Table showing the different conditions for stress behaviors at the end of the string.

curves with finite stress at the ends.

\subsection{Transition of the stress at the ends of a curve}

We first consider the transition at the end $\theta_{1}^{*}$. We are moving along an iso- $\mathrm{T}$ line in the parameter space. We have chosen three points, as shown in figure 8.2 corresponding to the one end blow up region. Two of these points are on the either side of the stress boundary and one of them is on the stress boundary.
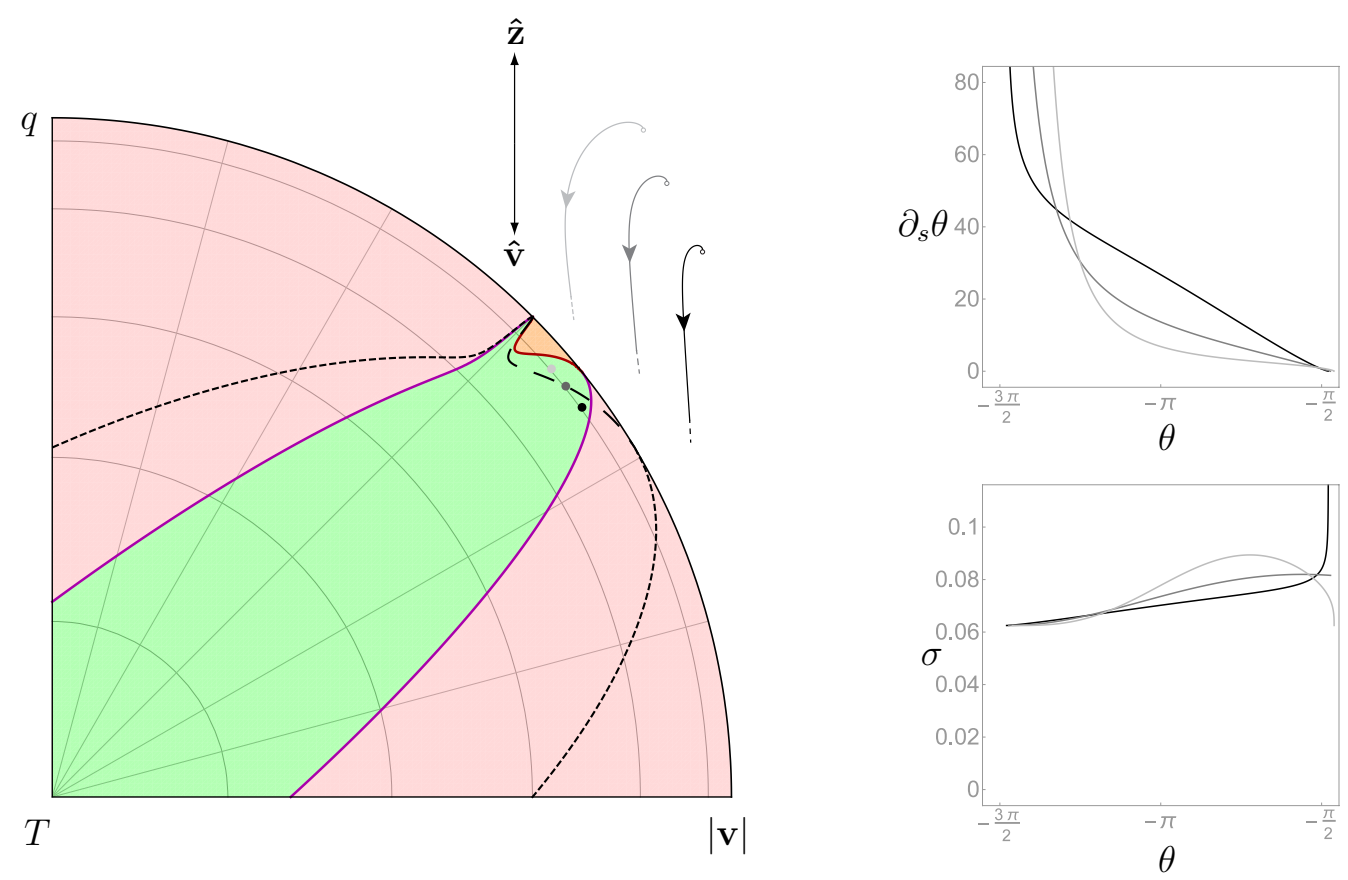

Figure 8.2: Orthographic projection of the parameter space and the curves corresponding to the three points (left). Orbits along with the stress distributions (right) for $R_{\nu}=0.6$. Here, $T=0.25 ;|\mathbf{v}|=0.74,0.76,0.78 ; q=0.63,0.60,0.57 ; m=1$. The angle of translation $\phi=-89^{\circ}$. 
The stress at one end $\left(\theta_{2}^{*}\right)$ of the curve is always equal to $m T^{2}$. The stress on the other end goes through a transition. For the points on the either side of the dashed boundary it is $m T^{2}$ for one case and infinite for the other. However, the stress tends to a finite value for the curve on the dashed boundary. This value is provided in appendix D.

The important point that we want to highlight is that even though the configurations of the curves are very similar to each other, their stress behavior is significantly different. We also see that how a semi-infinite curve can have either finite and infinite stress at the end.

Probably more bewildering is the next behavior, when we cross the other boundary that governs the stress at the trailing end of the string. We see a very similar transition as above. We find that in presence of axial flow infinitely long curves can have finite stress at one end.
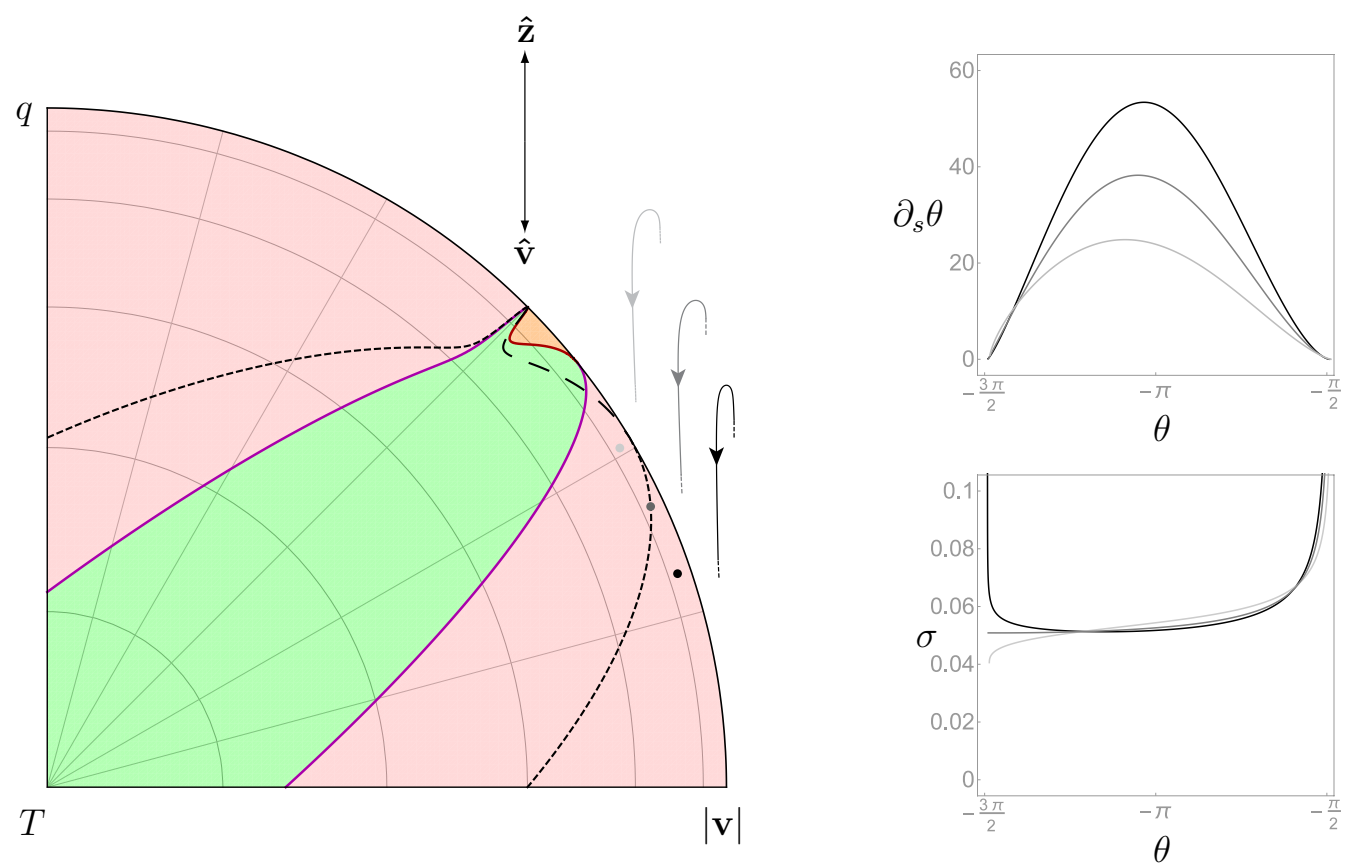

Figure 8.3: Orthographic projection of the parameter space and the curves corresponding to the three points (left). Orbits along with the stress distributions (right) for $R_{\nu}=0.6$. Here, $T=0.2 ;|\mathbf{v}|=0.84,0.88,0.93 ; q=0.49,0.41,0.30 ; m=1$. The angle of translation $\phi=-89^{\circ}$. 


\section{Chapter 9}

\section{Boundary value problems}

We have so far looked at the geometry of planar curves arising from different types of phase portraits. We have not specified any particular boundary conditions for the problem. In this chapter we want to study equilibrium shapes of a string when the boundary conditions have been specified.

\subsection{Towing of strings of fixed lengths}

Consider the problem of towing a string of fixed length, with fixed end points, through a fluid at uniform velocity. Or it may be that the string is fixed between two end points and there is uniform flow past the string. What is the equilibrium shape of the string? A problem like this can come up in low Reynolds number flow past bacterial biofilms [64].

In many natural aquatic systems, bacteria are found in self-organized sessile communities known as biofilms [64]. In the presence of a flow, biofilms often develop into long filamentous structures called streamers. These flows are inherently at low Reynolds number. The equilibrium shapes of streamers in a flow is an interesting question that is related to our problem. One can also think of other problems, like equilibrium shapes of cables that are being towed by ships from two ends. However this problem is at higher Reynolds number where the drag forces vary non-linearly with velocity [52].

\subsubsection{Finding the plane containing the curve}

In chapter 2 we discussed how the generic solutions to the 3D problem are planar. The string is being towed in three dimensions, however it always lies in a plane. In this section 
we discuss how to identify the plane containing the string.

We start by looking at the force balance in the binormal direction for the general problem given by eqn (2.5) and obtain,

$$
\frac{\hat{\mathbf{v}} \cdot \hat{\mathbf{b}}}{\hat{\overline{\mathbf{q}} \cdot \hat{\mathbf{b}}}}=\frac{|\overline{\mathbf{q}}|}{\nu_{\perp}|\overline{\mathbf{v}}|}
$$

The hat denotes a unit vector. Let the angle between the velocity vector and the binormal vector be $\alpha$. Similarly we call the angle between the body force vector and the binormal vector $\beta$. Then we have, $\hat{\overline{\mathbf{v}}} \cdot \hat{\mathbf{b}}=\cos \alpha$ and $\hat{\mathbf{q}} \cdot \hat{\mathbf{b}}=\cos \beta$. We also write, $\frac{|\overline{\mathbf{q}}|}{\nu_{\perp}|\overline{\mathbf{v}}|}=M$. Then the above relation can be written as:

$$
\frac{\cos \alpha}{\cos \beta}=M
$$

A plane can be specified uniquely by specifying the unit normal to it. The binormal vector is the unit normal to the plane in which the string is contained. The vector joining the end points of the string, $\mathbf{R}$ must also lie in the plane of the curve. This condition uniquely specifies the binormal vector.

The three vectors $\{\hat{\overline{\mathbf{q}}}, \hat{\overline{\mathbf{v}}}, \hat{\mathbf{R}}\}$ span our three dimensional space, and we shall describe the problem in terms of these three vectors. These are respectively the unit vectors for the body force, velocity and the position vector joining end points of the string. It must be remembered that these vectors are in general not orthogonal to each other. The binormal vector needs to be orthogonal to the vector $\hat{\mathbf{R}}$ and we know it is a unit vector. Also, the binormal has to maintain a certain angle between the body force and the velocity, as specified in (9.2). These things can be written out in the following three equations:

$$
\begin{aligned}
\hat{\mathbf{b}} \cdot \hat{\mathbf{R}} & =0 \\
\hat{\mathbf{b}} & =\cos \beta \hat{\overline{\mathbf{q}}}+\cos \alpha \hat{\overline{\mathbf{v}}}+b_{R} \hat{\mathbf{R}} \\
\hat{\mathbf{b}} \cdot \hat{\mathbf{b}} & =1
\end{aligned}
$$

For a given problem we will have information about the three vectors spanning our space. Once these three vectors are specified one can solve the above equations to determine the binormal vector uniquely.

Now we are required to find the projections of the velocity and the body force on the plane 
of the curve. We shall also like to define two orthogonal axes $\hat{\mathbf{x}}, \hat{\mathbf{z}}$ that will span the plane of the curve. Let us first find the projection of the body force on the plane. This is given by,

$$
\mathbf{q}=\overline{\mathbf{q}}-\overline{\mathbf{q}} \cdot \hat{\mathbf{b}} \hat{\mathbf{b}}
$$

We call the direction of this projected body force the negative $\hat{\mathbf{z}}$ direction, to be consistent with our formulation in section 3.1. So the $\hat{\mathbf{z}}$ axis in the plane of the curve can be defined as follows:

$$
\hat{\mathbf{z}}=-\frac{\mathbf{q}}{|\mathbf{q}|}
$$

The other axis $\hat{\mathbf{x}}$ is orthonormal to $\hat{\mathbf{z}}$ and can be found by the use of the following facts:

- $\hat{\mathrm{x}}$ is normal to $\hat{\mathrm{z}}$. So we have,

$$
\hat{\mathbf{x}} \cdot \hat{\mathbf{z}}=0
$$

- Since $\hat{\mathbf{x}}$ is on the plane containing the curve, it must be normal to the binormal vector. That is:

$$
\hat{\mathbf{x}} \cdot \hat{\mathbf{b}}=0
$$

- And we know that $\hat{\mathbf{x}}$ is unit vector. So,

$$
\hat{\mathbf{x}} \cdot \hat{\mathbf{x}}=1
$$

Using the above three conditions one can solve for $\hat{\mathbf{x}}$.

The vector $\mathbf{R}$ joining the end points of the string is contained in the plane of the curve. So we can write this vector in terms of $\hat{\mathbf{x}}$ and $\hat{\mathbf{z}}$ in the following way:

$$
\begin{aligned}
|R| \hat{\mathbf{R}} & =R_{x} \hat{\mathbf{x}}+R_{z} \hat{\mathbf{z}} \\
R_{x} & =|R| \hat{\mathbf{R}} \cdot \hat{\mathbf{x}} \\
R_{z} & =|R| \hat{\mathbf{R}} \cdot \hat{\mathbf{z}}
\end{aligned}
$$

The projected velocity in the plane is given by: 


$$
\mathbf{v}=\overline{\mathbf{v}}-\overline{\mathbf{v}} \cdot \hat{\mathbf{b}} \hat{\mathbf{b}}
$$

The angle $\phi$, defined in the figure 3.1 can now be calculated:

$$
\cos \phi=\frac{\hat{\mathbf{x}} \cdot \mathbf{v}}{|\mathbf{v}|}
$$

So now we have reduced our 3D problem onto the plane of the curve. We have the projected body force and the velocity in the plane. These projected velocities are dimensional quantities. The scale used to nondimensionalize velocity will be the following:

$$
\text { Velocity } \sim \sqrt{\mathbf{v} \cdot \mathbf{v}+T^{2}+\left(\frac{|\mathbf{q}|}{\nu_{\perp}}\right)^{2}}=\Gamma
$$

If we now scale our projected quantities by the above scale we get the nondimensionalized variables which lie on $\mathbb{S}^{2}$.

\subsubsection{Curves in 3D}

In the previous section, we have outlined that given the body force, the end points of a string and the velocity of translation, we can identify the plane in which the string will lie. In this section we do an example problem where we identify the plane of the curve and study the effect of changing the magnitude of the velocity of translation.

We are showing in figure 9.1 below a curve of fixed length that is being towed by its end points in a fluid. The direction of translation of the curve is shown in the figure by the velocity vector. The darker curves indicate a higher velocity of translation. It is observed that as the magnitude of translation velocity increases the plane of the curve makes larger angles with the $\hat{\mathbf{q}}-\hat{\mathbf{R}}$ plane. 

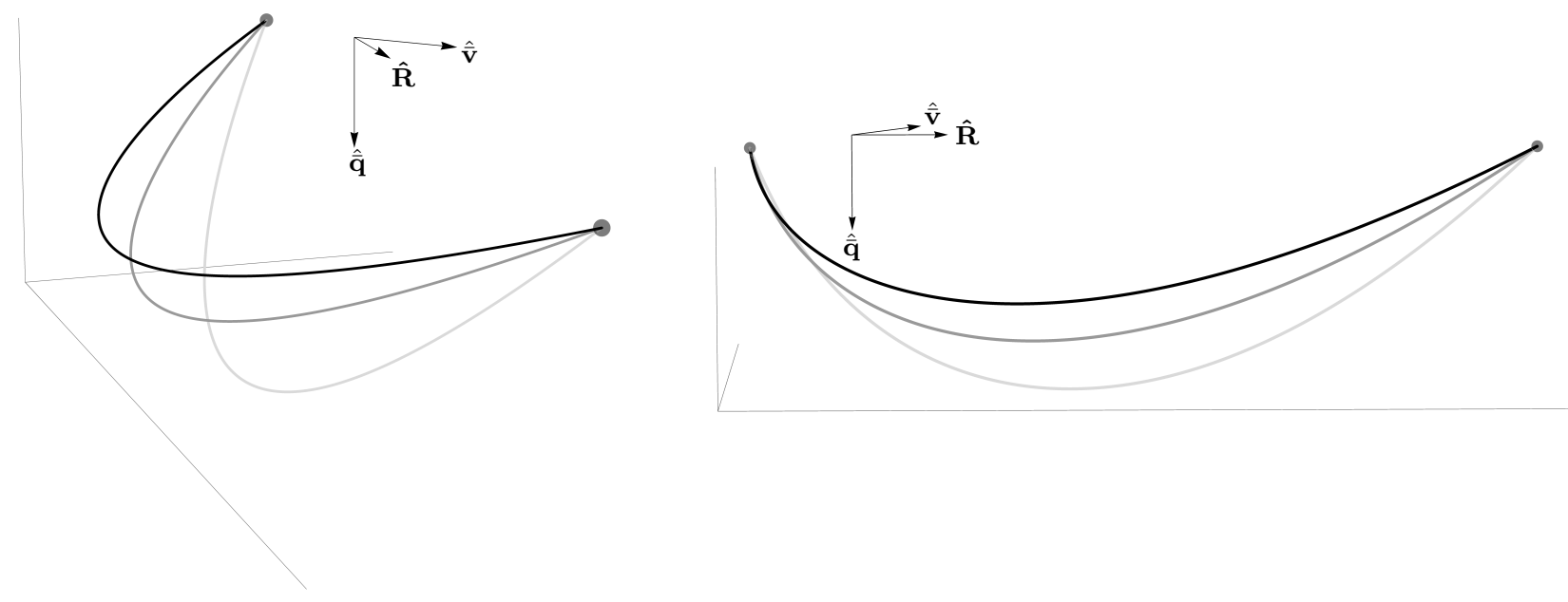

Figure 9.1: The figure shows two perspectives of how the plane containing the curve changes in $3 \mathrm{D}$ when it is towed from its end points with different velocities. Darker curves mean higher magnitude of velocity of translation. The parameters for the problem are: body force, $\overline{\mathbf{q}}=4$; velocities, $\overline{\mathbf{v}}=4,8,12$; the angle between the velocity vector and $\hat{\mathbf{R}}$ is $45^{\circ}$; the distance between end points, $|\mathbf{R}|=0.8$; normal drag coefficient $\nu_{\perp}=0.8$ and the tangential drag coefficient $\nu_{\|}=0.4$. The length of the curves are unity.

\subsubsection{Pure towing}

Consider a neutrally buoyant cable that is being towed in a fluid. This is a special case of our problem, where the body force $(\overline{\mathbf{q}})$ is zero. The projected normalized velocity on the plane of the curve will always be unity for this problem. Figure 9.2 shows three curves for different values of drag anisotropy $\left(R_{\nu}\right)$. 


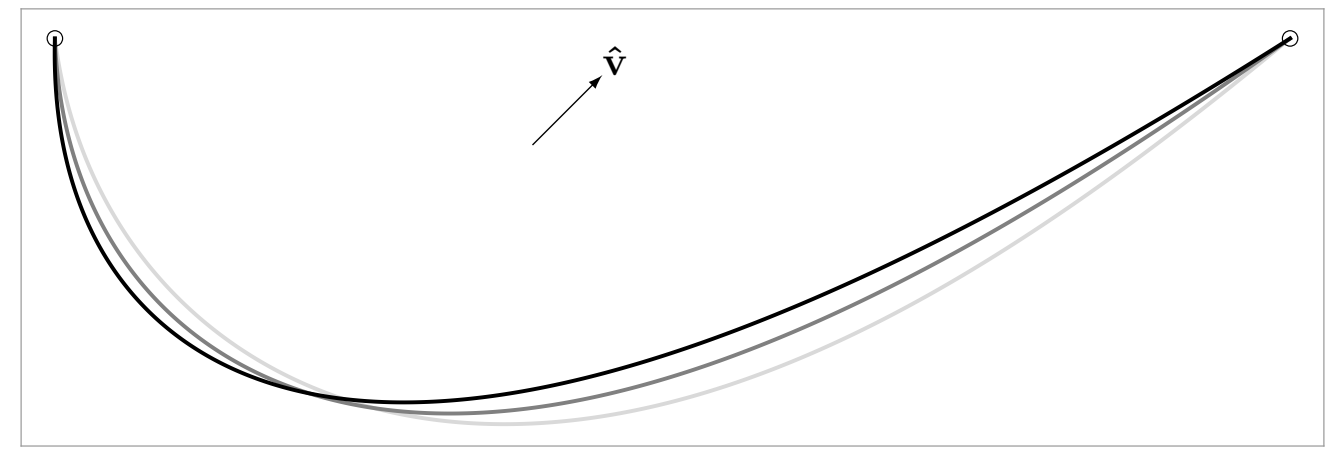

Figure 9.2: Shapes of towed strings for different anisotropy of drag forces. The direction of the towing velocity is shown in the figure. The distance between the end points is 0.8 units and the length of the curve is unity. Darker curves mean higher values of $R_{\nu}$ (more isotropy in drag forces). Here $R_{\nu}=0,0.5,1$.

Since there is no body force in the plane of the curve, it is always oriented in the direction of translation. However, the anisotropy in the drag forces affect the shape of the curves. This can be seen from figure 9.2.

\subsection{Pure axial flow: reeling in and out}

Let us consider a string of a given length that is being held between two fixed points, with an axial flow or tangential velocity in the string. This can be realized if it is reeled in from one end and reeled out from the other. In section 3.3 we said that if the body force is zero, then this problem does not have any equilibrium solutions other than the special case of a straight line. However if the body force is nonzero, there are other equilibrium solutions that we discuss in this section.

We shall also like to point out that the shape of the catenary does not change on addition of axial flow in the absence of drag forces. However in the presence of drag forces one obtains different equilibrium solutions depending on the magnitude of the axial flow. This special case of our problem has potential applications in the problems of laying and recovery of cables in the ocean. The drag forces in those problems will be nonlinear with velocity, in contrast to our linear drag forces. However the following formulation provides a basis for extending the study to higher Reynolds number. 


\subsubsection{Formulation}

In this problem, the string is held fixed between two points and there is axial flow and body force. Since there is no translation of the string, the only drag force is in the tangential direction. When we obtained our first order dynamical, system shown in eqn (3.15) we used $\nu_{\perp}$ to obtain nondimensionalized variables. However, since there is no motion of the string in the normal direction, we do not have a normal drag coefficient in our problem. As a result, we cannot obtain the limit of this problem from (3.15). So we start with the dimensional force balance equation:

$$
m T^{2} \partial_{s}^{2} \mathbf{X}+\nu_{\|} T \hat{\mathbf{t}}=\partial_{s}(\sigma \hat{\mathbf{t}})-q \hat{\mathbf{z}}
$$

For this problem we use the following scales to normalize:

$$
\begin{aligned}
\text { Length } & \sim L \\
\text { Velocity } & \sim \sqrt{T^{2}+\left(\frac{q}{\nu_{\|}}\right)^{2}}=\Gamma \\
\text { Force } & \sim L^{3} \nu_{\|} \Gamma \\
\text { Mass } & \sim \frac{L^{4} \nu_{\|}}{\Gamma}
\end{aligned}
$$

So our nondimensionalized variables become:

$$
\tilde{s}=\frac{s}{L} \quad \tilde{T}=\frac{T}{\Gamma} \quad \tilde{q}=\frac{q}{\nu_{\|} \Gamma} \quad \tilde{m}=\frac{m \Gamma}{L \nu_{\|}}
$$

Using our nondimensionalized variables and dropping the tilda like before for the sake of convenience, we get:

$$
m T^{2} \partial_{s}^{2} \mathbf{X}+T \hat{\mathbf{t}}=\partial_{s}(\sigma \hat{\mathbf{t}})-q \hat{\mathbf{z}}
$$

Note that because of our nondimensionalization the normalized tangential velocity and the body force lie on a circle $\left(\mathbb{S}^{1}\right)$,

$$
T^{2}+q^{2}=1
$$


Now we can project eqn (9.7) in the tangent and normal directions and proceed like we did earlier. After some algebraic manipulation, one will obtain the following for the phase portrait:

$$
\partial_{s} \theta=C(\cos \theta)^{2}\left[\frac{1-\tan \frac{\theta}{2}}{1+\tan \frac{\theta}{2}}\right]^{\frac{T}{q}}
$$

The above dynamical system can be written in a more compact form using trigonometric identities. However, we wanted to highlight the structure that it shows with our original dynamical system in eqn (3.15). Note that now we have a two parameter family of solutions, with the parameters being the axial flow $T$ and the scaling constant $C$.

The stress in the string is given by:

$$
\sigma=m T^{2}+\frac{q \cos \theta}{\partial_{s} \theta}
$$

Special cases: Just like our previous cases, here the phase portrait goes through a transition from heteroclinic connection to one end blow up. The transition occurs through a $2 \pi$ periodic special heteroclinic case at $\frac{T}{q}=0.2$.

We also have a transition in stress at the end of the string. When the axial flow is sufficiently small, we have stress going to infinity at both ends of the string. However there is a transition in stress behavior at $\frac{T}{q}=1$. After this transition, the stress at the trailing end of the string is finite and is equal to $m T^{2}$. The proof for these values is identical to that shown in appendix D.

\subsubsection{Equilibrium shapes}

Let us consider a string of length $L$. Let the distance between its end points from which it is reeled be $D$. Then it is seen that the shape of these curves at a given value of axial flow is governed by the parameter $\frac{L}{D}$. Here we show an example for a given $\frac{L}{D}$ ratio at different magnitudes of the tangential velocity. 


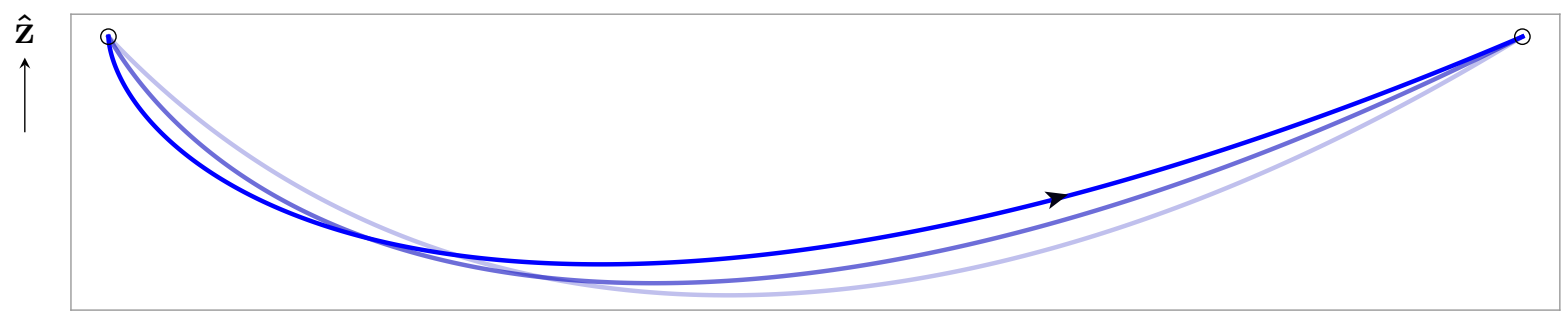

Figure 9.3: Equilibrium configurations are shown. Darker curves mean higher tangential velocity. The direction of the tangential velocity is shown in the figure. The parameters for the system are: length of the curves, $L=1$; distance between the end points, $D=0.92$; axial flow $T=0.7,0.88,0.931$; body force $q=0.71,0.47,0.365$.

The transition in the phase portrait from heteroclinic to one end blow up happens at $\frac{T}{q}=2$ or alternatively at $T=0.89$. We have provided configurations for curves both before and after this transition. From figure 9.3 we see that this transition does not affect the geometry of the curves significantly.

\subsubsection{Shapes for the special case}

Now we consider the special case of this problem when $\frac{T}{q}=2$. At this point one obtains $2 \pi$ periodic heteroclinic connections, like we obtained in section 7.2. The figure below shows two different curves with different $\frac{L}{D}$ ratios.
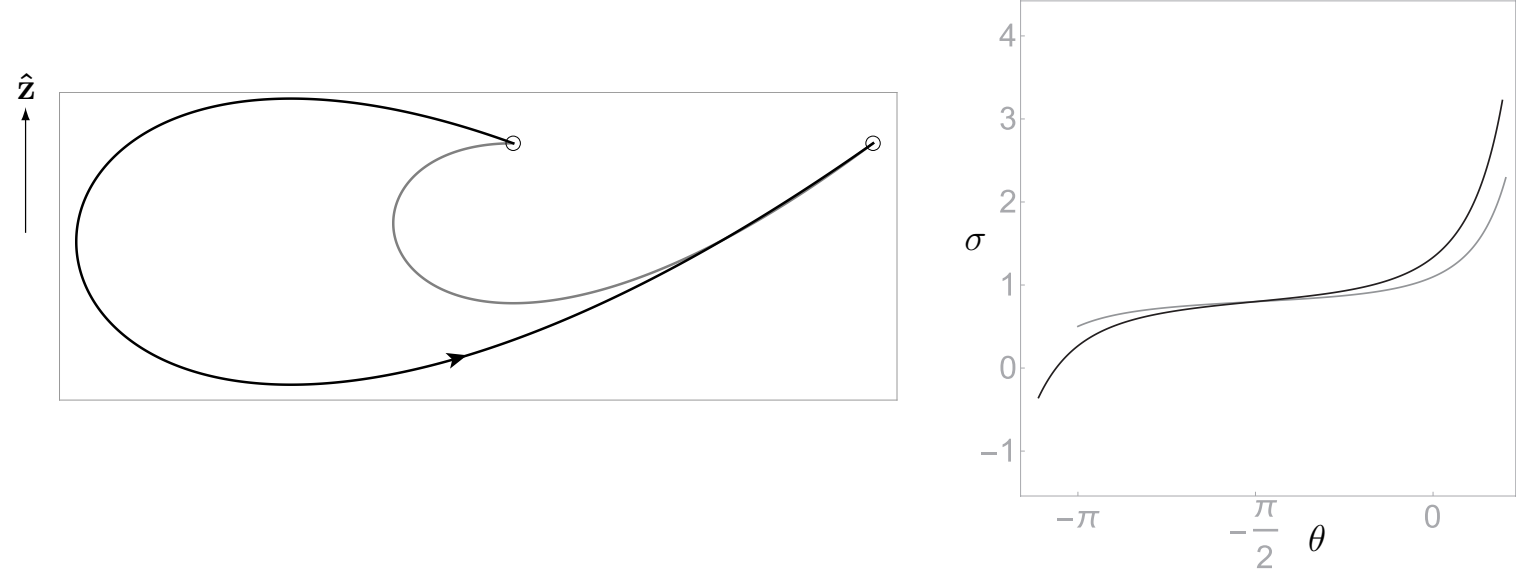

Figure 9.4: Equilibrium configurations of curves (left) for different $\frac{L}{D}$ ratios along with their stress distributions (right) are shown in the figure. These solutions are for the special case at the transition between heteroclinic connections and one end blow up phase portraits. Here $\frac{T}{q}=2 ; \frac{L}{D}=2,4 ; m=1$. 
From the stress distributions of the two curves, we observe that the curve with smaller $\frac{L}{D}$ is purely in tension. However, a portion of the curve with higher $\frac{L}{D}$ ratio is in compression. This suggests that these solutions are not stable. However, from the expression for stress provided in the eqn (9.9) we observe that if the mass density of the string is sufficiently high, one can obtain solutions that are purely in tension. So curves with high $\frac{L}{D}$ can be stabilized by making the mass density higher.

\subsubsection{Stress at the trailing end}

Figure 9.5 shows the stress distribution at the trailing end of a string for the case of pure axial flow in presence of body force. The dashed vertical line on the figure marks the point where there is a transition in stress. Note that the stress at the trailing end does not have a monotonic relation with $\frac{L}{D}$. This is also true for the catenary. We do not know what is the exact functional dependence of the stress at the trailing end with the tangential velocity and we are yet to understand the whole scenario.

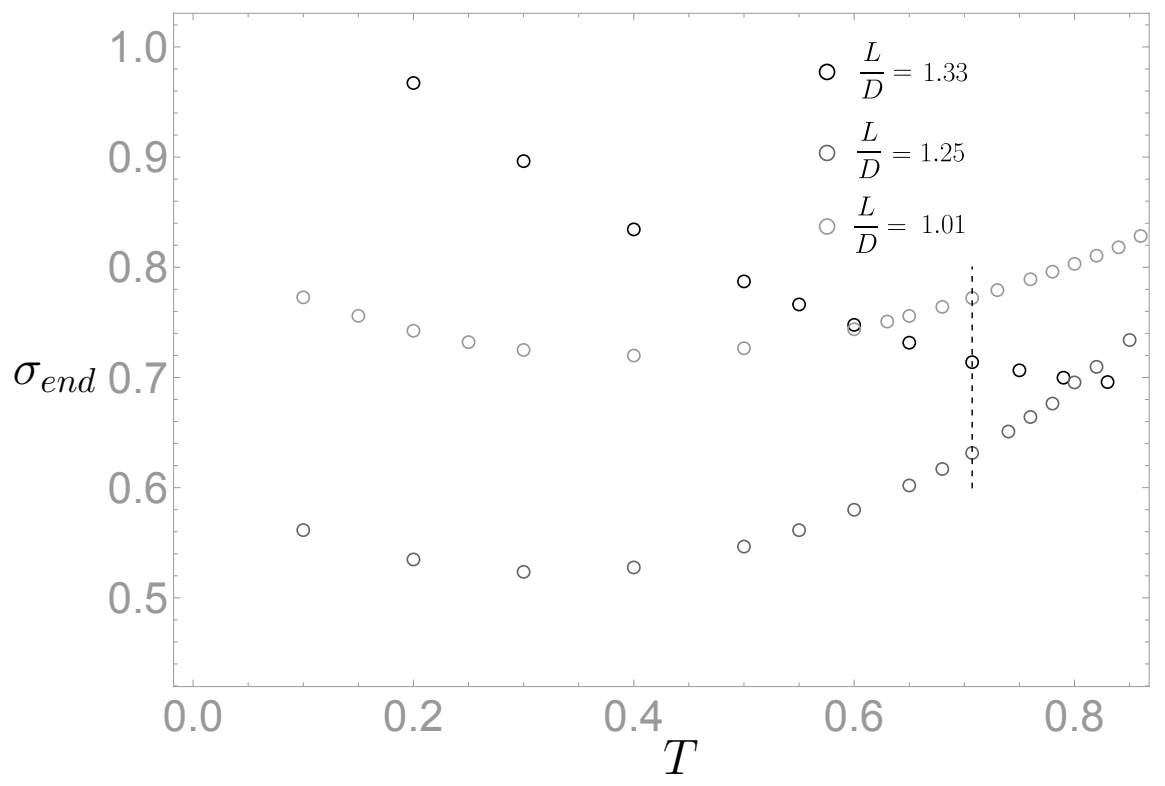

Figure 9.5: Stress at the trailing end of the string as a function of tangential velocity for different $\frac{L}{D}$ ratios and $m=1$. 


\section{Chapter 10}

\section{Discussion and future work}

The problem that we have studied so far can be divided in two parts. First we have looked at the geometry of a family of curves that satisfy a first order dynamical system. We also looked at the stress distribution for these curves. The solutions for finite length curves with zero stress at the ends correspond to sedimenting solutions for flexible filaments. Some of the obtained configurations where unstable due to the presence of compressive stresses.

Then we have discussed a set of problems where we have set the boundary conditions for a fixed length of string and looked at their equilibrium shapes under different conditions. We have discussed problems related to towing and reeling of a string.

The work in this thesis provides the basic framework to look at a class of these kinds of problems. In this chapter we want to discuss some of the aspects of our problem that we want to understand in a better way and some of the possible extensions that will help us to take on new problems.

Stress behavior: In chapter 8 we discussed how the stress at the end of the strings behaves in a counter-intuitive way. Some of our results showed that there can be solutions where infinitely long curves can translate with zero stress at one end. We do not yet understand these results completely. The behavior of the stress is also complicated when fixed end boundary conditions are specified. This was discussed in figure 9.5. We want to study these boundary value problems in further detail and understand how the transition in the stress or in the phase portraits affects the geometry of the strings that have fixed end points. In order to study this we feel that one needs to refine the single point shooting method by implementing multi-point (or parallel) shooting. This will allow one to explore a larger parameter space, that may shed light on this behavior. 
Non uniform flow: All the problems that we have discussed here consider uniform velocity of translation of the string. However it will be really interesting to study the equilibrium shape of a string that is in a non uniform flow. This will help us to study problems like the shapes of bacterial biofilms in micro-channels [64] or bending of elastic filaments in microfluidic applications [65]. So far these problems have been studied numerically. However it will be interesting to know if one can find exact solutions for these cases. Another question that may be asked is will it be always possible to reduce a general 3D problem onto a plane in case of a non uniform flow? One can start investigating these questions with canonical shear flows like plane Couette flow or Poiseuille flow.

Quadratic drag forces and experiments: We have considered only linear drag forces in this problem. Linear drag forces are inherent to low Reynolds number and are important in microfluidic problems. However, a challenging task will be to extend this formulation for quadratic drag forces that are observed at higher Reynolds number.

Zajac [52] provided exact solutions for the problems of towing and laying of submarine cables considering quadratic drag forces. In his work he considered purely flexible cables that are being laid from a ship moving with a uniform velocity onto the ocean bed with different topographies. The appropriate boundary condition suggested for the laying problem was either zero tension or zero tangential angle, at the end of the cable, at the bottom of the ocean. It was shown that a straight line is the only configuration that satisfies the zero tension boundary condition for the cable. However if the stress is finite at the end, then the tangential angle at the end of the cable is zero, and the equilibrium shape is given in terms of integrals that had to be evaluated numerically. For the range of velocities of operation, the normal drag force remains constant but the tangential drag may vary. However, often the tangential drag forces are negligible compared to the weight of the cable. If the tangential drag forces are neglected then it was possible to evaluate the integrals that determine the shape of the cable and a closed form solution for the shape of the cable was obtained. In the usual process of recovery of a cable, the tension at the ocean bed is nonzero and it is thought that there may not be any straight line solutions for the problem. The tension in the recovery process is usually much higher compared to laying of cables. Zajac further points out, that in general the cable is planar; however, if one accounts for the effect of oceanic currents then the cable configuration is not necessarily planar.

Besides the work of Zajac, Choo and Casarella [53] provided analytical models for the configurations of cables that are towed in circular path. Chapman [55] did a more comprehensive study of the tow cable system during ship turning by performing both dynamic and steady state simulations. In future, we want to come up with a general formulation for towing problems with quadratic drag forces and extend these solutions to more general cases. It 
will be interesting to look at the parameter space for nonlinear drag forces and find out an exact solution for a family of curves.

Incorporating quadratic drag forces in the formulation opens the door to table top experiments. Consider the example of the Lariat chain [66]. This is often seen in science exhibitions or as a toy. The Lariat chain is a loop of chain that hangs off, and is spun by a wheel. Due to the high speed of the rotating wheel, the chain is subjected to nonlinear drag forces. Extending the case of pure axial flow for quadratic drag forces will allow us to study these problems where inexpensive experiments can also complement the theory.

We believe that this work will in general inspire further research in the broad topic of dynamical equilibria of flexible objects. 


\section{Appendix A}

\section{Fixed Points}

In this chapter we show how to obtain the fixed points of our first order dynamical system given by eqn (3.15). We introduce a few notations for our convenience. We define:

$$
\begin{aligned}
\mathcal{F}(\theta) & =\left[\frac{1-\bar{U}-(\bar{W}+\bar{Q}) \tan \frac{\theta}{2}}{1+\bar{U}+(\bar{W}+\bar{Q}) \tan \frac{\theta}{2}}\right] \\
\psi(\theta) & =-\bar{U} \sin \theta+(\bar{W}+\bar{Q}) \cos \theta \\
k & =1+R_{\nu} \bar{U}^{2}+\left(R_{\nu} \bar{W}+\bar{Q}\right)(\bar{W}+\bar{Q})
\end{aligned}
$$

So we can write the first order dynamical system as:

$$
\partial_{s} \theta=C e^{\bar{U}\left(1-R_{\nu}\right) \bar{Q} \theta}|\mathcal{F}(\theta)|^{\bar{T}}|\psi(\theta)|^{k}
$$

Fixed points of this system are obtained by setting $\partial_{s} \theta$ to zero. So at the fixed points, we have:

$$
C e^{\bar{U}\left(1-R_{\nu}\right) \bar{Q} \theta}|\mathcal{F}(\theta)|^{\bar{T}}|\psi(\theta)|^{k}=0
$$

From which we either have $\mathcal{F}(\theta)=0$ or $\psi(\theta)=0$. Figure A.1 shows a phase portrait representing the primary lobe of the heteroclinic connections. Two fixed points are also marked in the figure. 


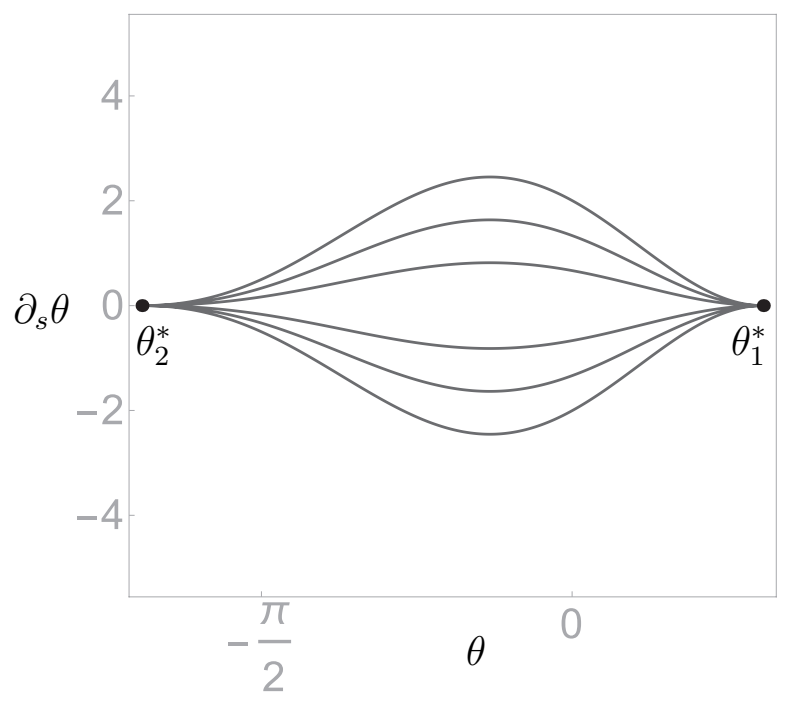

Figure A.1: Primary lobe of the heteroclinic connections along with the two fixed points are shown in the figure.

Let us first set $\psi$ to zero. On doing that we obtain:

$$
\begin{aligned}
\psi & =0 \\
\Longrightarrow \tan \theta & =\left(\frac{\bar{W}+\bar{Q}}{\bar{U}}\right) \\
\Longrightarrow \theta^{*} & =\tan ^{-1}\left(\frac{\bar{W}+\bar{Q}}{\bar{U}}\right)+n \pi \\
\text { Let } \theta_{1}^{*} & =\tan ^{-1}\left(\frac{\bar{W}+\bar{Q}}{\bar{U}}\right)
\end{aligned}
$$

Noting the general solution $\left(\theta^{*}\right)$ of the problem we can write, $\theta_{2}^{*}=\theta_{1}^{*}-\pi$.

The fixed points of the dynamical system can also be obtained by setting $\mathcal{F}$ to zero. On doing that one will obtain the following relationship:

$$
\theta^{*}=2 \tan ^{-1}\left(\frac{1-\bar{U}}{\bar{W}+\bar{Q}}\right)
$$

Now we shall like to show that this fixed point is equivalent to that obtained by setting $\psi$ to zero. For this first note that due to rescaling of our variables we have the relation: 


$$
\bar{U}^{2}+(\bar{W}+\bar{Q})^{2}=1
$$

Following the above relation we can write

$$
\begin{aligned}
(\bar{W}+\bar{Q})^{2} & =(1-\bar{U})(1+\bar{U}) \\
\Longrightarrow \frac{\bar{W}+\bar{Q}}{1+\bar{U}} & =\frac{1-\bar{U}}{\bar{W}+\bar{Q}}
\end{aligned}
$$

Using the above relation we can write the following:

$$
\theta^{*}=2 \tan ^{-1}\left(\frac{\bar{W}+\bar{Q}}{1+\bar{U}}\right)
$$

We now use a trig identity for half angles:

$$
\tan ^{-1} x=2 \tan ^{-1} \frac{x}{1+\sqrt{1+x^{2}}}
$$

We already have an expression for $\theta_{1}^{*}$ from (A.4). Using that expression we substitute $\frac{\bar{W}+\bar{Q}}{\bar{U}}$ in place of $x$ of the above identity. If we use the relation relating the rescaled quantities then after some simplification we obtain:

$$
\tan ^{-1}\left(\frac{\bar{W}+\bar{Q}}{\bar{U}}\right)=2 \tan ^{-1}\left(\frac{\bar{W}+\bar{Q}}{1+\bar{U}}\right)
$$

Now using (A.4) we can conclude that the fixed point obtained from $\psi=0$ is equivalent to that obtained from $\mathcal{F}=0$. So the two fixed points for the primary lobe as defined in section 3.2 are given by:

$$
\begin{aligned}
& \theta_{1}^{*}=\tan ^{-1}\left(\frac{\bar{W}+\bar{Q}}{\bar{U}}\right) \\
& \theta_{2}^{*}=\theta_{1}^{*}-\pi
\end{aligned}
$$




\section{Appendix B}

\section{Scaling between adjacent lobes}

In section 3.2 we have discussed that our phase trajectories are all $\pi$ periodic if appropriate scaling and reflection operations are used. Here we derive the scaling relation provided in eqn (3.16).

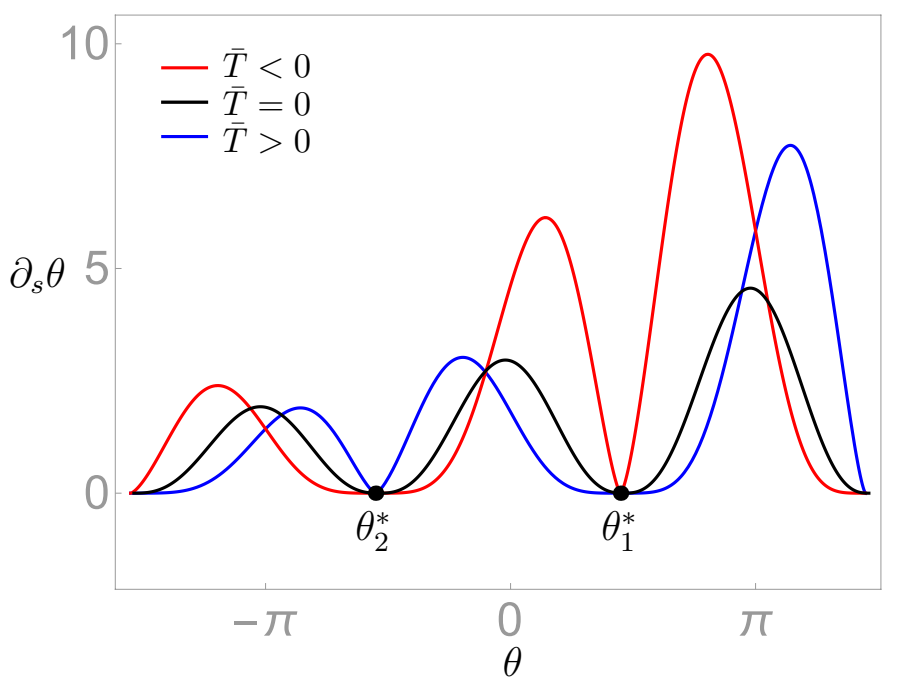

In order to discuss this we have re-produced figure 3.3 on the left. We have already defined:

$\mathcal{F}(\theta)=\left[\frac{1-\bar{U}-(\bar{W}+\bar{Q}) \tan \frac{\theta}{2}}{1+\bar{U}+(\bar{W}+\bar{Q}) \tan \frac{\theta}{2}}\right]$

Due to this function the orbits have different shape for positive and negative direction of the axial flow. We also had:

Figure B.1: Orbits of heteroclinic connections in presence and absence of axial flow.

$$
\theta_{1}^{*}=2 \tan ^{-1}\left(\frac{1-\bar{U}}{\bar{W}+\bar{Q}}\right)
$$

Using the above expression for our fixed point we can show that: 


$$
\begin{aligned}
& \mathcal{F}\left(\theta_{1}^{*}+\theta\right)=\frac{\bar{U}-1}{W+Q} \tan \frac{\theta}{2} \\
& \mathcal{F}\left(\theta_{2}^{*}+\theta\right)=\frac{1-\bar{U}}{W+Q} \cot \frac{\theta}{2}
\end{aligned}
$$

Now we multiply eqn (B.1) with (B.2) and use the fact that $\bar{U}^{2}+(\bar{W}+\bar{Q})^{2}=1$ to obtain:

$$
\mathcal{F}\left(\theta_{1}^{*}+\theta\right) \mathcal{F}\left(\theta_{2}^{*}+\theta\right)=\frac{\bar{U}-1}{\bar{U}+1}
$$

The above relation can be further modified to write the following:

$$
\left|\frac{\bar{U}+1}{\bar{U}-1} \mathcal{F}\left(\theta_{1}^{*}+\theta\right)\right|^{\bar{T}}=\left|\mathcal{F}\left(\theta_{2}^{*}+\theta\right)\right|^{-\bar{T}}
$$

We already know that the function $\psi$ is $\pi$ periodic. And we note:

$$
e^{\bar{Q} \bar{U}\left(1-R_{\nu}\right)\left(\theta_{1}^{*}+\theta\right)}=e^{\bar{Q} \bar{U}\left(1-R_{\nu}\right)\left(\theta_{2}^{*}+\pi+\theta\right)}
$$

Now we recall from our discussion in section 3.2 that in order to obtain similar curves from adjacent lobes one needs to perform a rotation on the integrated curves. This corresponds to a change of sign in the scaling constant that results in a reflection of the orbits in the phase space about the horizontal plane. Using the above relations and accounting for the change of sign in the scaling constant, we can write:

$$
C \partial_{s} \theta\left(\theta_{2}^{*}+\theta,-\bar{T}\right) \equiv-C\left(e^{-\bar{Q} \bar{U}\left(1-R_{\nu}\right) \pi}\left|\frac{\bar{U}+1}{\bar{U}-1}\right|^{\bar{T}}\right) \partial_{s} \theta\left(\theta_{1}^{*}+\theta,+\bar{T}\right)
$$

This is the relation that we established in (3.16). In absence of axial flow all the lobes are only scaled by the exponential term of the eqn (B.5) and there is only one reflection operation about the horizontal plane in the phase space. 


\section{Appendix C}

\section{On conditions for different phase portraits}

In section 3.2 we discuss that depending upon the choice of parameters we can have three different kinds of phase portraits. They are: heteroclinic connections, two end blow up and one end blow up. In this chapter we discuss that what are the conditions for these different kinds of phase portraits.

We already know that our first order dynamical system is of the following form:

$$
\partial_{s} \theta=C e^{\bar{U}\left(1-R_{\nu}\right) \bar{Q} \theta}|\mathcal{F}(\theta)|^{\bar{T}}|\psi(\theta)|^{k}
$$

where the functions have their usual meaning as defined in eqn (A.1). The figure shown beside portrays the behavior of the individual functions between the two fixed points. We have also shown the function $\mathcal{F}^{-1}$ in the figure as we will be using this in this chapter. We notice that the function $\mathcal{F}$ blows up at $\theta_{2}^{*}$. However we can obtain heteroclinic

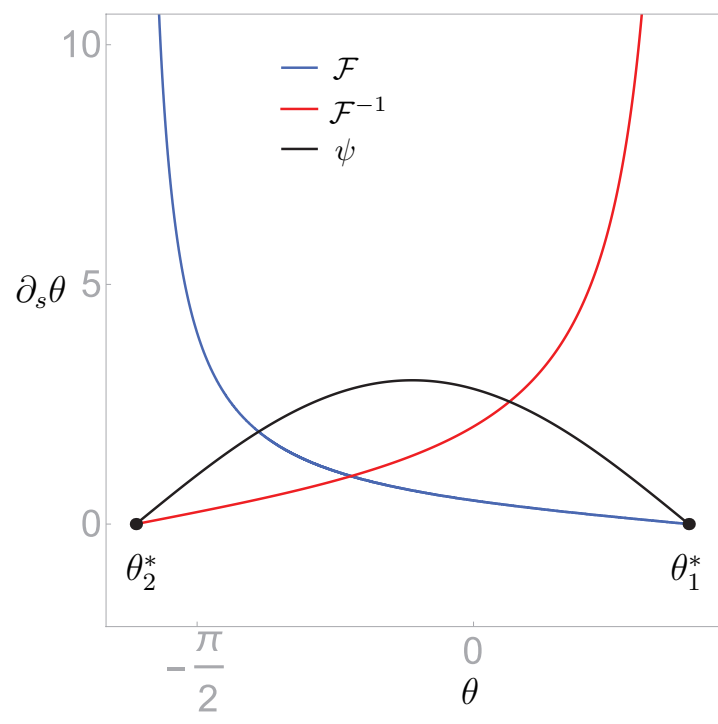

Figure C.1: Three functions connections as phase trajectories for this dynamical system which is zero at both the fixed points. The behavior of the phase trajectories depend on the magnitudes of the exponents of our first order dynamical system.

In order to find the exact conditions for the different behaviors we first linearize the functions 
near the fixed points.

\section{C.1 Linearized Functions}

We want to understand the behavior of $\partial_{s} \theta$ at the two fixed points. In order to do so we need to calculate several limits using the linearized functions. For this purpose we linearize both $\mathcal{F}$ and $\mathcal{F}^{-1}$ about $\theta_{1}^{*}$ and $\theta_{2}^{*}$ respectively. We denote all the linearized functions by a superscript star. On linearizion we obtain:

$$
\begin{aligned}
\left.\mathcal{F}^{*}\right|_{\theta_{1}^{*}} & =-\frac{1-\bar{U}}{2(\bar{Q}+\bar{W})}\left(\theta-\theta_{1}^{*}\right)+O\left(\theta-\theta_{1}^{*}\right)^{2} \\
\left.\mathcal{F}^{-1^{*}}\right|_{\theta_{2}^{*}} & =\frac{\bar{Q}+\bar{W}}{2(1-\bar{U})}\left(\theta-\theta_{2}^{*}\right)+O\left(\theta-\theta_{2}^{*}\right)^{2}
\end{aligned}
$$

We also linearize $\psi$ about both the fixed points to obtain:

$$
\begin{aligned}
& \left.\psi^{*}\right|_{\theta_{1}^{*}}=-\left(\theta-\theta_{1}^{*}\right)+O\left(\theta-\theta_{1}^{*}\right)^{2} \\
& \left.\psi^{*}\right|_{\theta_{2}^{*}}=\left(\theta-\theta_{2}^{*}\right)+O\left(\theta-\theta_{2}^{*}\right)^{2}
\end{aligned}
$$

\section{C.2 Limits and phase portrait behavior}

Now we focus on deriving the relationship between the exponents of the dynamical system for different kinds of behaviors.

Behavior at $\theta_{1}^{*}$ : We are interested to evaluate the following limit:

$$
L_{1}=\lim _{\theta \rightarrow \theta_{1}^{*}} \partial_{s} \theta
$$

In order to evaluate this limit let us look at the linearized form of the dynamical system. We substitute the linearized functions that we evaluated in the previous section from (C.2) 
and (C.4) to obtain:

$$
L_{1}=\lim _{\theta \rightarrow \theta_{1}^{*}}\left[C e^{\bar{U}\left(1-R_{\nu}\right) \bar{Q} \theta}\left|\frac{1-\bar{U}}{2(\bar{Q}+\bar{W})}\right|^{\bar{T}}\left(\theta_{1}^{*}-\theta\right)^{k+\bar{T}}\right]
$$

This limit blows up to infinity iff $k+\bar{T}<0$ and goes to zero if $k+\bar{T}>0$. However if $k+\bar{T}=0$ then we have a special case where the limit tends to a finite value. The finite limiting value is given by:

$$
M_{1}=C e^{\bar{U}\left(1-R_{\nu}\right) \bar{Q} \theta_{1}^{*}}\left|\frac{1-\bar{U}}{2(\bar{Q}+\bar{W})}\right|^{\bar{T}}
$$

Behavior at $\theta_{2}^{*}$ : Let us rewrite the expression for the dynamical system with a subtle difference:

$$
\partial_{s} \theta=C e^{\bar{U}\left(1-R_{\nu}\right) \bar{Q} \theta} \frac{|\psi|^{k}}{\left|\mathcal{F}^{-1}\right|^{\bar{T}}}
$$

We want to evaluate the limit of the dynamical system at $\theta_{2}^{*}$. That is the reason we wrote down the dynamical system in the preceding way. Note that now it is in a $\frac{0}{0}$ form. So we want to evaluate the following limit:

$$
L_{2}=\lim _{\theta \rightarrow \theta_{2}^{*}} \partial_{s} \theta
$$

Proceeding similarly with linearized functions we obtain:

$$
L_{2}=\lim _{\theta \rightarrow \theta_{2}^{*}}\left[C e^{\bar{U}\left(1-R_{\nu}\right) \bar{Q} \theta}\left|\frac{2(1-\bar{U})}{\bar{Q}+\bar{W}}\right|^{\bar{T}}\left(\theta-\theta_{2}^{*}\right)^{k-\bar{T}}\right]
$$

This limit goes to zero iff $k-\bar{T}>0$ and blows up if $k-\bar{T}<0$. However when $k-\bar{T}=0$ we have a special case where the limit is neither zero nor blowing up instead it tends to a finite value. That finite value is given by: 


$$
M_{2}=C e^{\bar{U}\left(1-R_{\nu}\right) \bar{Q} \theta_{2}^{*}}\left|\frac{2(1-\bar{U})}{\bar{Q}+\bar{W}}\right|^{\bar{T}}
$$

This completes our derivation for the different conditions on the exponents for different behaviors of the phase portrait. 


\section{Appendix D}

\section{Stress at the end of the string}

In this chapter we look at the stress behavior at the end of the cables or strings. In chapter 8 , we discussed that how the stress at the end of a string can behave in a counter intuitive way. Here we follow a very similar procedure as the previous chapter of the appendix and provide conditions on different kinds of stress behavior in the system.

\section{D.1 The origin of the strange behavior}

From equation (3.7) it follows that the stress distribution in the string is given by:

$$
\sigma=m T^{2}+\frac{-\mathbf{v} \cdot \hat{\mathbf{x}} \sin \theta+(\mathbf{v} \cdot \hat{\mathbf{z}}+q) \cos \theta}{\partial_{s} \theta}
$$

The above equation can be rewritten as:

$$
\sigma=m T^{2}+\frac{\psi \Delta}{\partial_{s} \theta}
$$

where,

$$
\begin{gathered}
\psi=-\bar{U} \sin \theta+(\bar{W}+\bar{Q}) \cos \theta \\
\Delta=\sqrt{(\mathbf{v} \cdot \hat{\mathbf{x}})^{2}+(\mathbf{v} \cdot \hat{\mathbf{z}}+q)^{2}}
\end{gathered}
$$


In order to help the reader figure (C.1) has been reproduced here. Figure C.1 shows the behavior of the functions that constitute the dynamical system. The functions have their usual meaning as defined in eqn (A.1).

We see from these functions that the behavior of the term $\frac{\psi}{\partial_{s} \theta}$ in eqn (D.1) is not trivial. Depending on different choices of parameters $\partial_{s} \theta$ can be zero (or can blow up) at the two fixed points (or poles) of the dynamical system. As a result of this the stress at the end of the string may also be finite or may be infinite. It probably comes as no surprise that the behavior of the stress is also governed by the exponents of the different terms in the dy-

namical system. Here the relation among the exponents is derived.

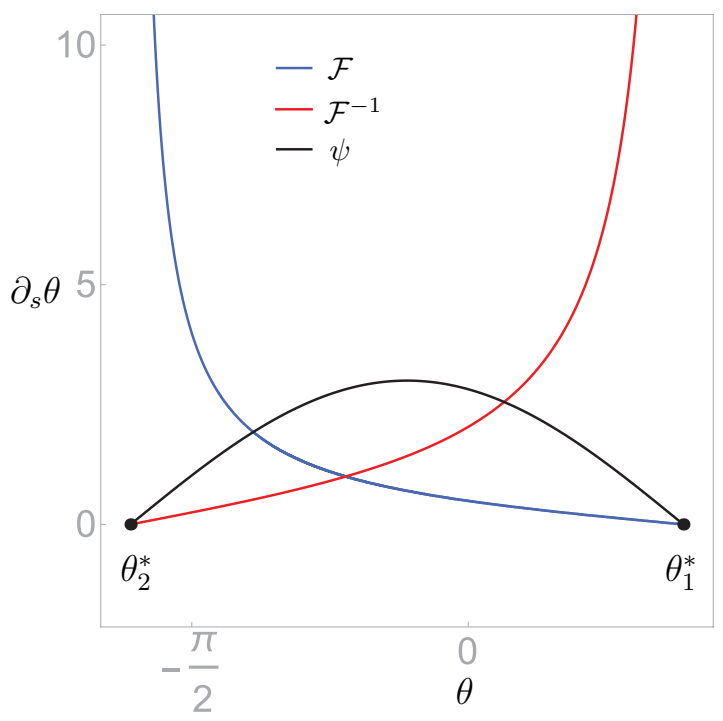

\section{D.2 Relations governing the stress}

In order to find the relation among the exponents that governs the behavior of the stress at the end points of the string, we take the same approach of evaluating limits with linearized functions. In order to do so, let us first write equation (D.1) in the following way:

$$
\sigma=m T^{2}+\frac{\psi \Delta}{C e^{\bar{U}\left(1-R_{\nu}\right) \bar{Q} \theta}|\mathcal{F}(\theta)|^{\bar{T}}|\psi(\theta)|^{k}}
$$

We have written out the expression for $\partial_{s} \theta$ in a similar way as in previous chapters of the appendix. Now let us evaluate the limit.

Stress at $\theta_{1}^{*}$ : In order to understand the behavior at the end we want to evaluate the following limit:

$$
L_{\sigma 1}=\lim _{\theta \rightarrow \theta_{1}^{*}} \sigma
$$

Looking at (D.2) we see that at $\theta_{1}^{*}$ the limit is of the form $\frac{0}{0}$. So we go ahead and linearize the functions in order to evaluate the limit. We already have the linearized functions given by (C.1)-(C.3) from previous chapter and on substituting in D.2 we get: 


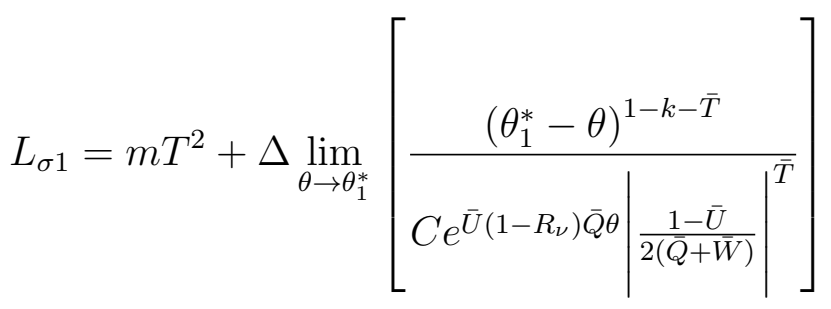

From the above expression we get that the limit tends to zero when the exponent, $1-k-\bar{T}$ is positive. The limit blows up when the exponent is negative and tends to a finite value indicating a special case when it is exactly zero. The limiting value of stress for the special case is given by:

$$
S_{1}=m T^{2}+\Delta \frac{e^{-\bar{U}\left(1-R_{\nu}\right) \bar{Q} \theta_{1}^{*}}}{C}\left|\frac{2(\bar{Q}+\bar{W}}{1-\bar{U}}\right|^{\bar{T}}
$$

Behavior at the end $\theta_{2}^{*}$ : The process of evaluation of this limit is very similar to what we have done above. We rewrite eqn (D.2) in a different way in order to have a $\frac{0}{0}$ form.

$$
\sigma=m T^{2}+\frac{\left|\mathcal{F}^{-1}\right| \bar{T} \psi \Delta}{C e^{\bar{U}\left(1-R_{\nu}\right) \bar{Q} \theta}|\psi|^{k}}
$$

So we are now interested to evaluate the limit:

$$
L_{\sigma 2}=\lim _{\theta \rightarrow \theta_{2}^{*}} \sigma
$$

In order to evaluate the limit we substitute the linearized function from (C.2)-(C.4) and obtain:

$$
L_{\sigma 2}=m T^{2}+\Delta \lim _{\theta \rightarrow \theta_{2}^{*}}\left[\frac{\left(\theta-\theta_{2}^{*}\right)^{1-k+\bar{T}}}{C e^{\bar{U}\left(1-R_{\nu}\right) \bar{Q} \theta}}\left|\frac{\bar{Q}+\bar{W}}{2(1-\bar{U})}\right|^{\bar{T}}\right]
$$

This limit goes to zero if the exponent, $1-k+\bar{T}$ is positive. This blows up if the exponent is negative and tends to a finite limiting value if the exponent is exactly zero which corresponds to a special case. The limit for the special case is given by: 


$$
S_{2}=m T^{2}+\Delta \frac{e^{-\bar{U}\left(1-R_{\nu}\right) \bar{Q} \theta_{2}^{*}}}{C}\left|\frac{\bar{Q}+\bar{W}}{2(1-\bar{U})}\right|^{\bar{T}}
$$

This completes our derivations on the relations of the exponents that determine the stress behavior at the end of the string. The discussion on these behaviors and plots is in chapter 8. 


\section{Bibliography}

[1] George Shoobridge Carr. A synopsis of elementary results in pure mathematics. Hodgson (London), 1886.

[2] Lisa Jardine. Monuments and microscopes: Scientific thinking on a grand scale in the early royal society. Notes and Records of the Royal Society, 55(2):289-308, 2001.

[3] Amir R Alexander. Duel at dawn: heroes, martyrs, and the rise of modern mathematics, volume 22. Harvard University Press, 2010.

[4] Thomas Jefferson, Kaveh Azar, McDowall, and JP Boyd. Papers of Thomas Jefferson, Volume 19: January 1791 to March 1791. Princeton University Press, 1974.

[5] Thomas Jefferson. Memoirs, correspondence and private papers of Thomas Jefferson, late President of the United States, volume 2. Colburn and Bentley, 1829.

[6] John Joseph Fahie. Galileo, his life and work. J. Murray, 1903.

[7] Izrail Moiseevich Gelfand, Izrail Moiseevitch Gelfand, and Sergeŭ Vasilevich Fomin. Calculus of variations. Courier Corporation, 2000.

[8] Edward John Routh. A treatise on analytical statics: with numerous examples, volume 1. Cambridge University Press, 2013.

[9] WH Thomson. On machinery for laying submarine telegraph cables. The Engineer, 4:185-187, 1857.

[10] Edward M Purcell. Life at low Reynolds number. Am. J. Phys, 45(1):3-11, 1977.

[11] Eric Lauga and Thomas R Powers. The hydrodynamics of swimming microorganisms. Reports on Progress in Physics, 72(9):096601, 2009.

[12] Geoffrey Taylor. Analysis of the swimming of microscopic organisms. Proceedings of the Royal Society of London. Series A. Mathematical and Physical Sciences, 209(1099):447461, 1951. 
[13] GJ Hancock. The self-propulsion of microscopic organisms through liquids. Proceedings of the Royal Society of London. Series A. Mathematical and Physical Sciences, 217(1128):96-121, 1953.

[14] J Gray and GJ Hancock. The propulsion of sea-urchin spermatozoa. Journal of Experimental Biology, 32(4):802-814, 1955.

[15] Stuart F Goldstein and Nyles W Charon. Multiple-exposure photographic analysis of a motile spirochete. Proceedings of the National Academy of Sciences, 87(13):4895-4899, 1990.

[16] Paul D Frymier, Roseanne M Ford, Howard C Berg, and Peter T Cummings. Threedimensional tracking of motile bacteria near a solid planar surface. Proceedings of the National Academy of Sciences, 92(13):6195-6199, 1995.

[17] CM Pooley, GP Alexander, and JM Yeomans. Hydrodynamic interaction between two swimmers at low Reynolds number. Physical review letters, 99(22):228103, 2007.

[18] Sunghwan Jung, Kathleen Mareck, Lisa Fauci, and Michael J Shelley. Rotational dynamics of a superhelix towed in a stokes fluid. Physics of Fluids (1994-present), 19(10):103105, 2007.

[19] Alfred Shapere and Frank Wilczek. Geometry of self-propulsion at low Reynolds number. Journal of Fluid Mechanics, 198:557-585, 1989.

[20] Henry C Fu, Thomas R Powers, and Charles W Wolgemuth. Theory of swimming filaments in viscoelastic media. Physical review letters, 99(25):258101, 2007.

[21] Allison P Berke, Linda Turner, Howard C Berg, and Eric Lauga. Hydrodynamic attraction of swimming microorganisms by surfaces. Physical Review Letters, 101(3):038102, 2008 .

[22] Gwynn J Elfring and Eric Lauga. Hydrodynamic phase locking of swimming microorganisms. Physical review letters, 103(8):088101, 2009.

[23] Thomas R Powers. Dynamics of filaments and membranes in a viscous fluid. Reviews of Modern Physics, 82(2):1607, 2010.

[24] C Lemaitre, Pascal Hémon, and Emmanuel De Langre. Instability of a long ribbon hanging in axial air flow. Journal of Fluids and Structures, 20(7):913-925, 2005.

[25] C. Grouthier, S. Michelin, Y. Modarres-Sadeghi, and E. de Langre. Self-similar vortexinduced vibrations of a hanging string. Journal of Fluid Mechanics, 724, 62013. 
[26] Médéric Argentina and L Mahadevan. Fluid-flow-induced flutter of a flag. Proceedings of the National academy of Sciences of the United States of America, 102(6):1829-1834, 2005.

[27] Sebastien Michelin, Stefan G Llewellyn Smith, and Beverley J Glover. Vortex shedding model of a flapping flag. Journal of Fluid Mechanics, 617:1-10, 2008.

[28] Silas Alben and Michael J Shelley. Flapping states of a flag in an inviscid fluid: bistability and the transition to chaos. Physical review letters, 100(7):074301, 2008.

[29] Carl M Larsen and Karl H Halse. Comparison of models for vortex induced vibrations of slender marine structures. Marine Structures, 10(6):413-441, 1997.

[30] Matteo Luca Facchinetti, Emmanuel De Langre, and Francis Biolley. Coupling of structure and wake oscillators in vortex-induced vibrations. Journal of Fluids and structures, 19(2):123-140, 2004.

[31] Olivier Doaré and Sébastien Michelin. Piezoelectric coupling in energy-harvesting fluttering flexible plates: linear stability analysis and conversion efficiency. Journal of Fluids and Structures, 27(8):1357-1375, 2011.

[32] Clement Grouthier, Sebastien Michelin, and Emmanuel de Langre. Energy harvesting using vortex-induced vibrations of tensioned cables. arXiv preprint arXiv:1203.0236, 2012.

[33] Emmanuel De Langre. Effects of wind on plants. Annu. Rev. Fluid Mech., 40:141-168, 2008.

[34] Thomas Barois and Emmanuel De Langre. Flexible body with drag independent of the flow velocity. Journal of Fluid Mechanics, 735:R2, 2013.

[35] Diego Lopez, Christophe Eloy, Sébastien Michelin, and Emmanuel de Langre. Drag reduction, from bending to pruning. EPL (Europhysics Letters), 108(4):48002, 2014.

[36] Edward John Routh. The advanced part of a treatise on the dynamics of a system of rigid bodies. 1905.

[37] Bruno H Zimm. Dynamics of polymer molecules in dilute solution: viscoelasticity, flow birefringence and dielectric loss. The journal of chemical physics, 24(2):269-278, 1956.

[38] Chris H Wiggins and Raymond E Goldstein. Flexive and propulsive dynamics of elastica at low Reynolds number. Physical Review Letters, 80(17):3879, 1998. 
[39] Y-N Young and Michael J Shelley. Stretch-coil transition and transport of fibers in cellular flows. Physical review letters, 99(5):058303, 2007.

[40] John Happel and Howard Brenner. Low Reynolds number hydrodynamics: with special applications to particulate media, volume 1. Springer Science \& Business Media, 1983.

[41] Xianghua Xu and Ali Nadim. Deformation and orientation of an elastic slender body sedimenting in a viscous liquid. Physics of Fluids (1994-present), 6(9):2889-2893, 1994.

[42] RG Cox. The motion of long slender bodies in a viscous fluid part 1. general theory. Journal of Fluid mechanics, 44(04):791-810, 1970.

[43] X Schlagberger and RR Netz. Orientation of elastic rods in homogeneous stokes flow. EPL (Europhysics Letters), 70(1):129, 2005.

[44] M Cosentino Lagomarsino, I Pagonabarraga, and CP Lowe. Hydrodynamic induced deformation and orientation of a microscopic elastic filament. Physical review letters, 94(14):148104, 2005.

[45] Anna-Karin Tornberg and Michael J Shelley. Simulating the dynamics and interactions of flexible fibers in stokes flows. Journal of Computational Physics, 196(1):8-40, 2004.

[46] Isaac Llopis, Ignacio Pagonabarraga, M Cosentino Lagomarsino, and Christopher P Lowe. Sedimentation of pairs of hydrodynamically interacting semiflexible filaments. Physical Review E, 76(6):061901, 2007.

[47] Lei Li, Harishankar Manikantan, David Saintillan, and Saverio E Spagnolie. The sedimentation of flexible filaments. Journal of Fluid Mechanics, 735:705-736, 2013.

[48] George Biddell Airy. I. on the mechanical conditions of the deposit of a submarine cable: To the editors of the philosophical magazine and journal. 1858.

[49] William Thomson. 1884, on the forces concerned in the laying and lifting of deep-sea cables. Mathematical and Physical Papers, 2:153À167, 1865.

[50] Leonard Pode. An experimental investigation of the forces on stranded cables. Technical report, DTIC Document, 1950.

[51] Leonard Pode. Tables for computing the equilibrium configuration of a flexible cable in a uniform stream. Technical report, DTIC Document, 1951.

[52] EE Zajac. Dynamics and kinematics of the laying and recovery of submarine cable. The Bell System Technical Journal, 36(5):1129-1207, 1957. 
[53] Young-Il Choc and Mario J Casarella. Configuration of a towline attached to a vehicle moving in a circular path. Journal of Hydronautics, 6(1):51-57, 1972.

[54] Young-il Choo and Mario J Casarella. A survey of analytical methods for dynamic simulation of cable-body systems. Journal of Hydronautics, 7(4):137-144, 1973.

[55] DA Chapman. Towed cable behaviour during ship turning manoeuvers. Ocean Engineering, 11(4):327-361, 1984.

[56] AP Dowling. The dynamics of towed flexible cylinders part 1. neutrally buoyant elements. Journal of Fluid Mechanics, 187:507-532, 1988.

[57] AP Dowling. The dynamics of towed flexible cylinders part 2. negatively buoyant elements. Journal of Fluid Mechanics, 187:533-571, 1988.

[58] Jiaming $\mathrm{Wu}$ and Allen T Chwang. A hydrodynamic model of a two-part underwater towed system. Ocean engineering, 27(5):455-472, 2000.

[59] Paul Williams and Pavel Trivailo. Periodic solutions for flexible cable-body systems towed in circular and elliptical paths. In AIAA Atmospheric Flight Mechanics Conference, 2006.

[60] Paul Williams, Daniel Sgarioto, and Pavel Trivailo. Optimal control of an aircraft-towed flexible cable system. Journal of guidance, control, and dynamics, 29(2):401-410, 2006.

[61] Mark A Grosenbaugh. Transient behavior of towed cable systems during ship turning maneuvers. Ocean Engineering, 34(11):1532-1542, 2007.

[62] Paul Williams and Pavel Trivailo. Dynamics of circularly towed aerial cable systems, part 2: Transitional flight and deployment control. Journal of guidance, control, and dynamics, 30(3):766-779, 2007.

[63] L Gary Leal. Advanced transport phenomena: fluid mechanics and convective transport processes. Cambridge University Press, 2007.

[64] Roberto Rusconi, Sigolene Lecuyer, Nicolas Autrusson, Laura Guglielmini, and Howard A Stone. Secondary flow as a mechanism for the formation of biofilm streamers. Biophysical journal, 100(6):1392-1399, 2011.

[65] Jason S Wexler, Philippe H Trinh, Helene Berthet, Nawal Quennouz, Olivia du Roure, Herbert E Huppert, Anke Lindner, and Howard A Stone. Bending of elastic fibres in viscous flows: the influence of confinement. Journal of Fluid Mechanics, 720:517-544, 2013. 
[66] Norman Tuck. Lariat chain, www.normantuck.com/catalogpages/lariat.html, 1986. 